\title{
A NOVEL APPROACH FOR ANALYZING MIXING QUALITY IN SOLID-LIQUID STIRRED TANKS VIA \\ COUPLED COMPUTATIONAL FLUID DYNAMICS - DISCRETE ELEMENT METHOD AND ELECTRICAL RESISTANCE TOMOGRAPHY
}

\author{
by \\ Cindy Tran \\ B.Eng in Chemical Engineering, Ryerson University, Toronto, Canada, 2015
}

\author{
A thesis \\ presented to Ryerson University \\ in partial fulfillment of the \\ requirements for the degree of \\ Master of Applied Science \\ in the Program of \\ Chemical Engineering \\ Toronto, Ontario, Canada, 2017 \\ (C) Cindy Tran 2017
}




\section{AUTHOR'S DECLARATION FOR ELECTRONIC SUBMISSION OF A THESIS}

I hereby declare that I am the sole author of this thesis. This is a true copy of the thesis, including any required final revisions, as accepted by my examiners.

I authorize Ryerson University to lend this thesis to other institutions or individuals for the purpose of scholarly research.

I further authorize Ryerson University to reproduce this thesis by photocopying or by other means, in total or in part, at the request of other institutions or individuals for the purpose of scholarly research.

I understand that my thesis may be made electronically available to the public. 


\section{Abstract}

\section{Cindy Tran}

\section{A Novel Approach for Analyzing Mixing Quality in Solid-Liquid Stirred Tanks via Coupled Computational Fluid Dynamics - Discrete Element Method and Electrical Resistance Tomography}

\section{Master of Applied Science, Chemical Engineering, Ryerson University, 2017}

The mixing quality of a solid-liquid stirred tank operating in the turbulent regime was investigated, numerically and to an extent experimentally. Simulations were performed by coupling Computational Fluid Dynamics (CFD) and the Discrete Element Method (DEM). The results were evaluated against experimental data obtained using Electrical Resistance Tomography (ERT). This facilitated a novel and more rigorous assessment of CFD-DEM coupling - i.e. based on the spatial distribution of particle concentrations. Furthermore, a new mixing index definition was developed to quantify suspension quality to work in tandem with existing dispersion mixing indexes. This provides a more complete interpretation of mixing quality. In this work, it was found that the model underestimated suspension and dispersion due to model limitations associated with mesh size and fluidparticle interaction models. Furthermore, the predicted mixing quality was sensitive to changes in the drag model, including other fluid-particle interaction forces in simulations, and variations in certain particle properties. 


\section{Acknowledgements}

I would like to sincerely express my gratitude to Dr. Farhad Ein-Mozaffari, Dr. Ali Lohi, and Dr. Mohammadreza Ebrahimi for their expert advice, continual guidance, and encouragement throughout this challenging project. It would not have been possible without their help.

I am also grateful to Ali Hemmati, Daniel Boothe, and Tondar Tajrobehkar for their technical expertise and support in the labs; the Chemical Engineering Department's staff and professors for their support with teaching assistantships and accessing resources; and my fellow researchers for their collaborative efforts and continual friendship throughout this thesis process.

Additionally, I would like to recognize the financial support of the Queen Elizabeth II Graduate Scholarship in Science and Technology (QEII-GSST), the Ryerson Graduate Fellowship (RGF), the Dr. Robert Guerriere Award, the teaching assistantships provided by the Chemical Engineering Department, and the graduate stipends provided by my supervisors. This research could not have been completed without it. 


\section{Table of Contents}

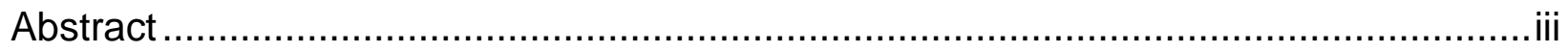

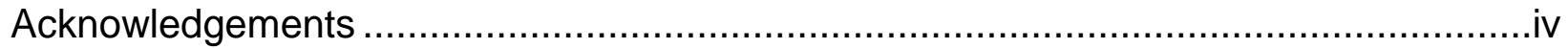

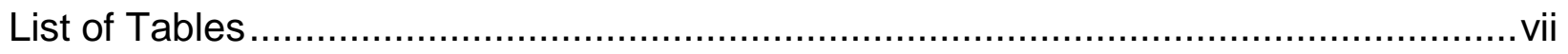

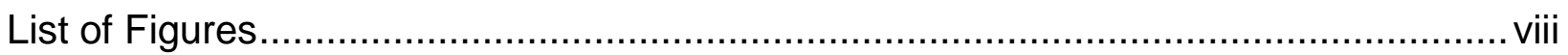

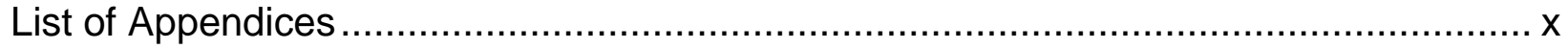

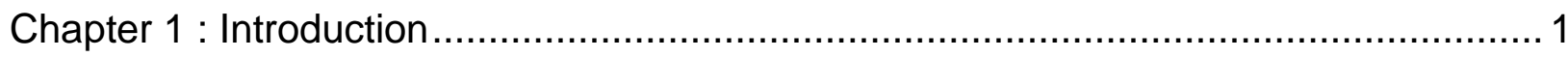

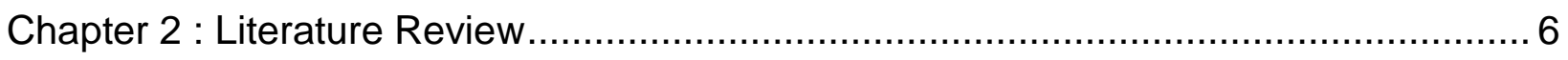

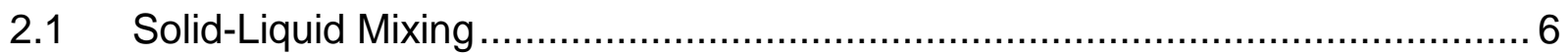

2.2 Theoretical Principles of Electrical Resistance Tomography (ERT) ................. 8

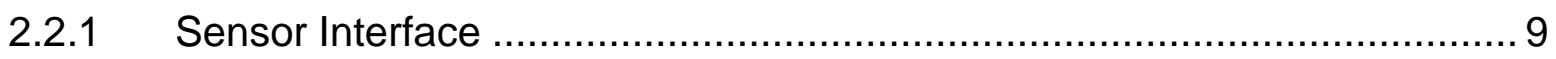

2.2.2 Data Acquisition System (DAS) ……........................................... 10

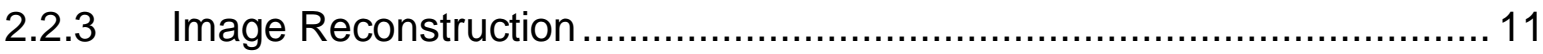

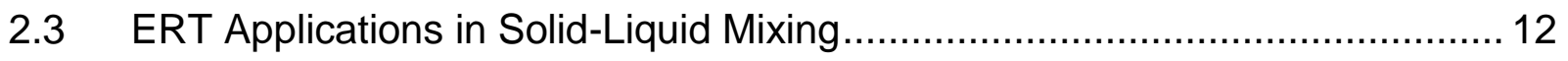

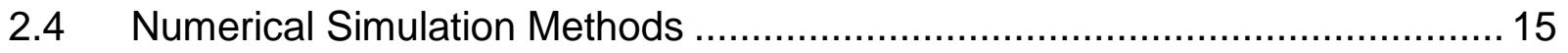

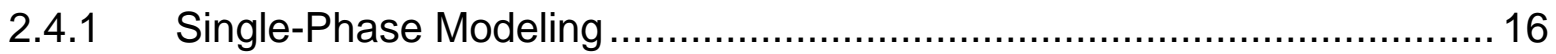

2.4.1.1 Lagrangian Models - Discrete Element Method (DEM) ...................... 16

2.4.1.2 Eulerian Models - Computational Fluid Dynamics (CFD) …................ 20

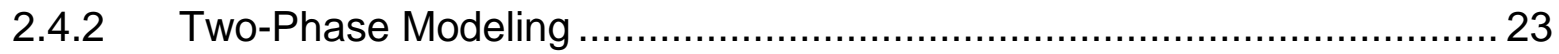

2.5 Theoretical Principles of Computation Fluid Dynamics (CFD) and Discrete Element Method (DEM) Coupling Model ............................................................... 25

2.5.1 General Overview of Governing Equation Forms …............................ 25

2.5.2 Modeling of Fluid-Particle Interaction Forces ...................................... 27

2.5.3 Coupling Approaches and Schemes................................................... 31

2.5.4 Mesh Size and Time Step Restrictions .............................................. 33

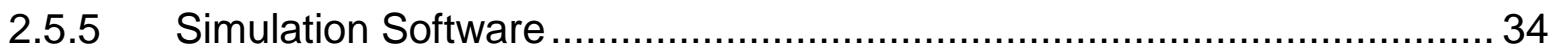

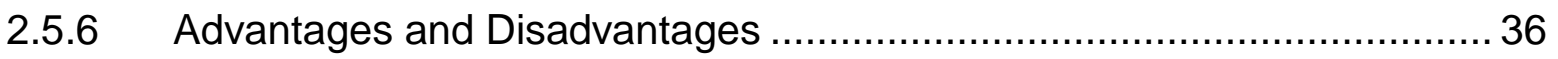

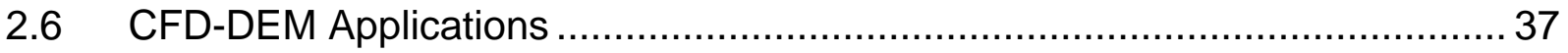

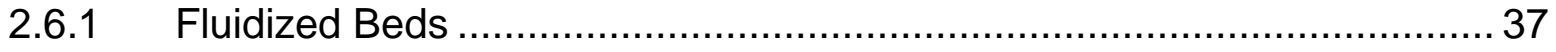

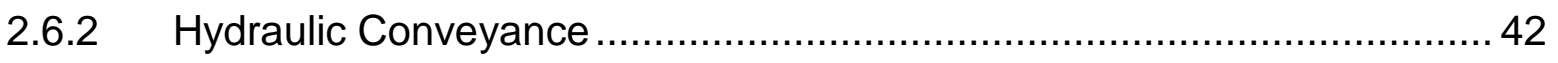




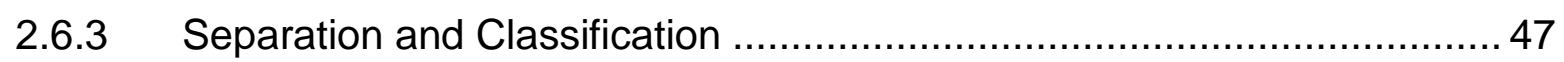

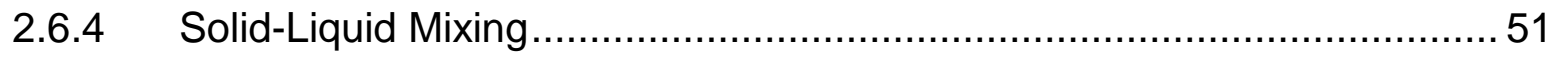

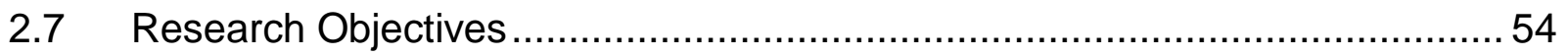

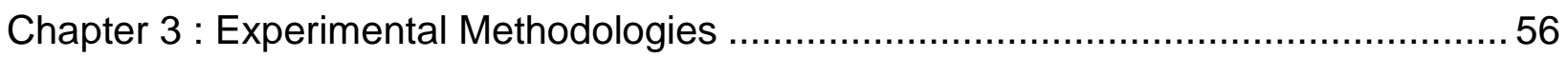

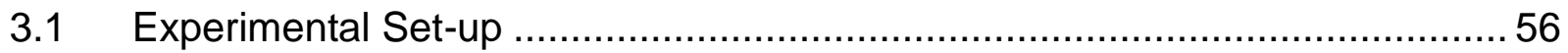

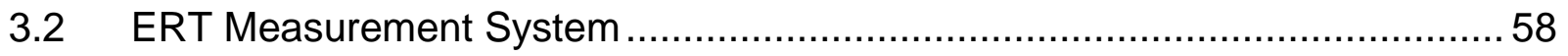

3.3 Quantification of Mixing Quality with Mixing Indexes .................................. 62

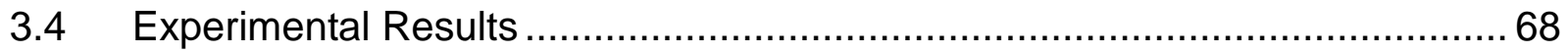

Chapter 4 : Computational Methodologies ………................................................... 70

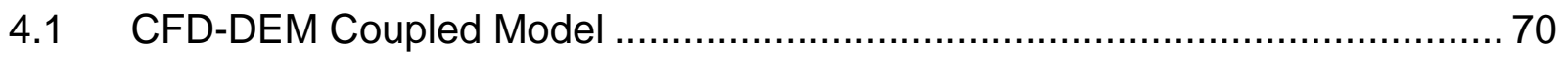

4.1.1 Governing Equations for Liquid Phase ………............................... 71

4.1.2 Governing Equations for Solid Phase …............................................ 72

4.1.3 Solid-Liquid Interaction Force Models ................................................. 74

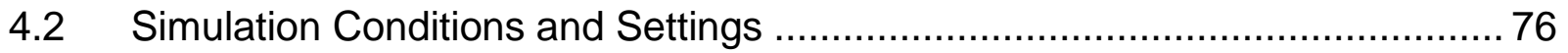

4.3 Fluid-Phase Mesh and Boundary Conditions used in CFD Solver .................. 78

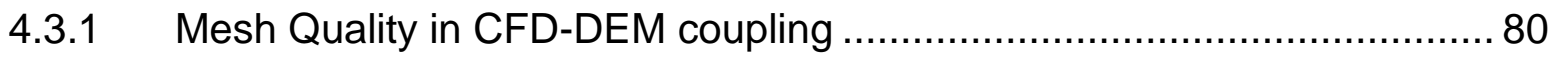

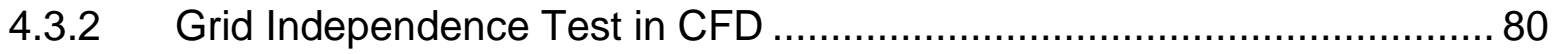

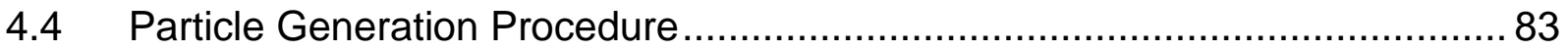

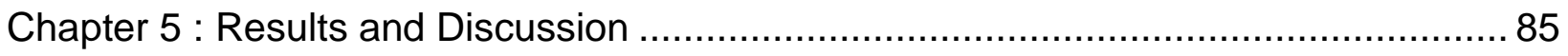

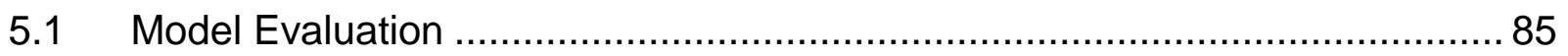

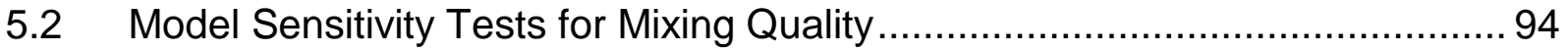

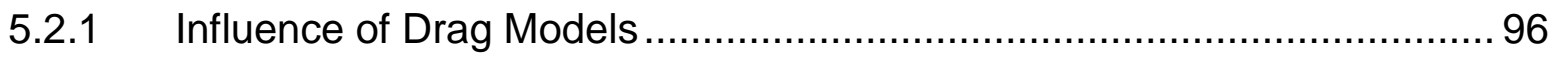

5.2.2 Influence of Other Fluid-particle Interaction Forces ............................... 98

5.2.3 Influence of Particle Properties ...................................................... 104

Chapter 6 : Conclusions and Recommendations ............................................... 112

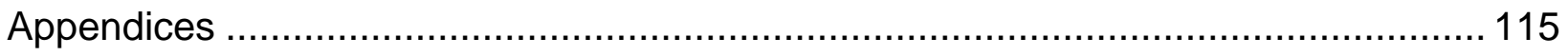

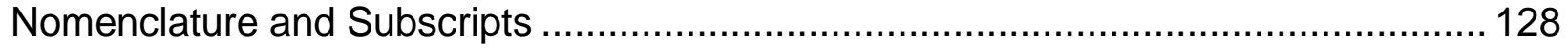

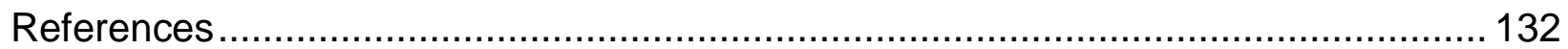




\section{List of Tables}

Table 1. Fluid-particle interaction forces definitions. ............................................... 28

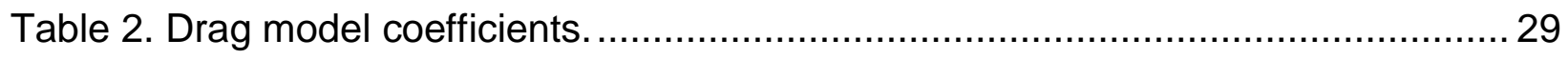

Table 3. Other fluid-particle interaction force models. ............................................... 30

Table 4. Experimental operating conditions. ....................................................... 58

Table 5. Settings used for ERT measurement. .......................................................... 59

Table 6. Summary of mixing indexes used previously in literature............................... 63

Table 7. Simulation parameters. ....................................................................... 77

Table 8. Summary of different cases for model sensitivity tests conducted at impeller

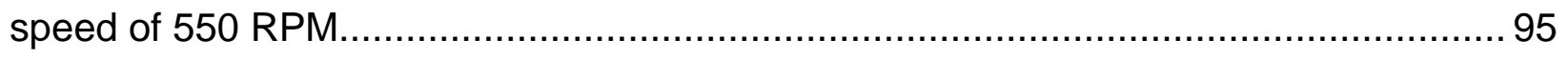

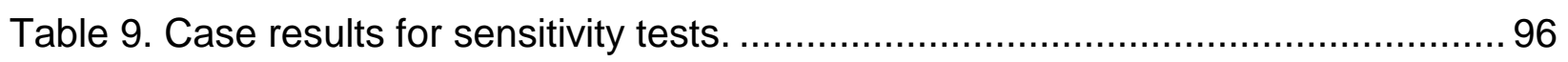




\section{List of Figures}

Figure 1. Contact diagrams for a) particle-particle and b) particle-wall contacts. .......... 18

Figure 2. Interphase coupling regime map [13] ...................................................... 31

Figure 3. Calculation cycle for coupling scheme [14] ........................................... 33

Figure 4. Schematic diagram of experimental set-up............................................. 57

Figure 5. ERT particle concentration tomographs - time-averaged for 30 frames - at

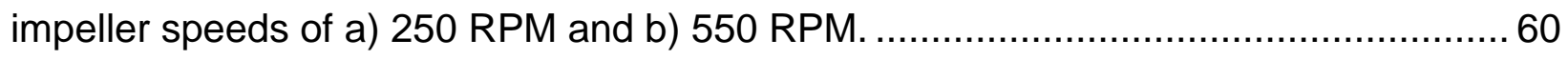

Figure 6. Quadrilateral ERT tomographic grid and zone scheme.............................. 65 Figure 7. Experimental suspension and dispersion mixing indexes - time-averaged for 30

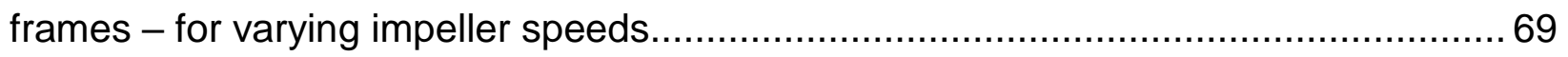

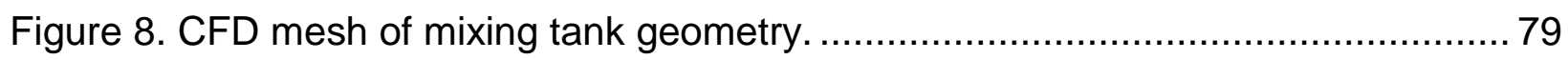
Figure 9. Comparison of time-averaged velocity profiles for grid-independent meshes along monitored line in liquid-only CFD simulations.

Figure 10. Comparison of time-averaged velocity profiles - along monitored line in liquidonly CFD simulations - for a) current mesh and grid-independent mesh b) current mesh with impeller speed of 625 RPM and grid-independent mesh with impeller speed of 550 RPM. 82

Figure 11. Particle generation images at a) a time shortly after $5 \mathrm{~s}$ b) a time between 5 and $6 \mathrm{~s}-$ but closer to $6 \mathrm{~s}-$ and $\mathrm{c}$ ) a time long after $6 \mathrm{~s}$.

Figure 12. Simulation images of instantaneous particle suspension pattern - taken after steady-state was reached. 86

Figure 13. Simulation images of a) time-averaged fluid flow field and b) axial profile of time-averaged particle volume fraction at impeller speed of 550 RPM. 87 Figure 14: Images of a) simulated time-averaged particle concentration planes and zoning scheme and b) comparison between experiment and simulation time-averaged local particle concentrations at impeller speed of 550 RPM. 89 Figure 15: Comparison of time-averaged local particle concentrations with impeller speed of 550 RPM for experiment and 625 RPM for simulation. 92 Figure 16. Simulation suspension and dispersion indexes for varying impeller speeds. 94 Figure 17. Comparison for the drag force model based on Cases 1A, 1B, and 1C...... 97 
Figure 18. Influence of various fluid-particle interaction forces on suspension and dispersion index differences and change in relative error based on Cases 1C, 2A, 2B, 2C,

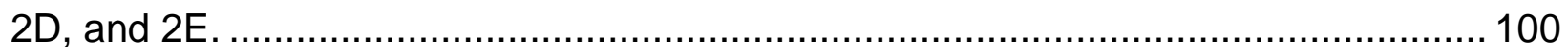

Figure 19. Influence of a) static friction coefficient - based on Cases 1C, 3A, and 3B and b) rolling friction coefficient - based on Cases 3B, 3C, 3D, and 3E - on suspension

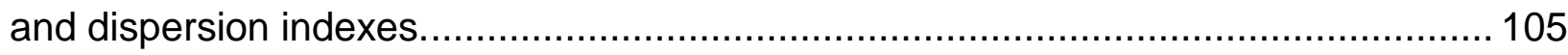
Figure 20. Influence of Young's Modulus on suspension and dispersion indexes based on Cases 3B, 3F, and 3G 109 Figure 21. Influence of coefficient of restitution on suspension and dispersion indexes based on Cases 3B, 3H, and 3I. 


\section{List of Appendices}

APPENDIX A. Di Felice User Defined Function ....................................................... 115

APPENDIX B. Magnus Lift Force User Defined Function........................................ 116

APPENDIX C. Modifications to Coupling Interface (Supplement to APPENDIX B) ..... 118

APPENDIX D. Basset Force User Defined Function .............................................. 119

APPENDIX E. Adjust User Defined Function (Supplement to APPENDIX D) .............. 122

APPENDIX F. Modifications to Coupling Interface (Supplement to APPENDIX D) ..... 123 


\section{Chapter 1 : Introduction}

Solid-liquid mixing is an essential component of many key processes used in the chemical engineering, food manufacturing, and pharmaceutical industries. Some processes include dispersion, dissolution, precipitation, adsorption, and solid-liquid reactions [1]. Due to its prominence, solid-liquid mixing has been the focus of a large body of research. The majority of this research has been conducted experimentally. This was achieved with a wide range of experimental methods, which varied not only in their measurement techniques but also in the parameters being measured. However, the current study will only focus on the measurement of the spatial distribution of particle concentrations. This has commonly been measured using x-ray, optical, ultrasound, and electrical tomography [2]. A particular case of the latter is referred to as Electrical Resistance Tomography (ERT). This method will be used in this study.

ERT has been applied to a multitude of unit operations such as mixers, reactors, digesters, separators, and filters [3]. However, for the sake of brevity, further discussion 
will be limited to applications involving solid-liquid mixing. Refer to Section 2.3 for the literature review of these applications, which also summarizes the various methods of analyzing tomographic data. Generally, the spatial distribution of the particle concentrations is summarized into a singular and easily comparable value referred to as a mixing index. However, a variety of mixing index definitions exist and are not consistently used. In the present study, the overall mixing index proposed by Harrison et al. [4] will be used. Refer to Section 3.3 for the reasoning behind this selection. It should be highlighted that the mixing index definitions - discussed in that section - only refer to particle dispersion within the measurement planes. This is quantified based on the standard deviation of the local particle concentrations. Alternatively, a new mixing index will be proposed in this work to evaluate the degree of particle suspension from the bottom of the tank. This will be quantified based on the average of the local particle concentrations.

Numerical simulations have emerged as a promising substitute for when performing experiments would be too expensive, unsafe, or even impossible. A variety of numerical methods can be used to simulate solid-liquid mixing. However, it has been conventionally modelled using the Two Fluid Method (TFM) [5]-[12]. This method uses Eulerian approaches to represents both the solid and liquid phases as continua. As a result, the computational intensity of the method is significantly reduced. However, this also reduces its accuracy. Since, the models used to account for particle-particle and fluid-particle interactions are currently limited in their application [13]. However, as technology advances, this trade-off between accuracy and computational intensity has become less necessary. Instead, more accurate and computationally intensive methods 
are becoming more practical. This is the case for the coupled Computational Fluid Dynamics and Discrete Element Method (CFD-DEM) approach. This model will be used in the present work.

In the coupled CFD-DEM approach, the solid phase is appropriately represented as discrete elements by using a Lagrangian approach. This results in better accuracy when capturing particle physics at the cost of a manageable increase in computational intensity [14]. The fluid flow is estimated by using the volume-averaged Navier-Stokes equations. In this unresolved modeling approach, models are still required to take fluidparticle interactions into consideration. These interactions are normally only taken into consideration in the fluid and particle momentum equations. Such that, the influence of particles on turbulence (i.e. turbulence modulation) is often neglected. The inaccuracy associated with the fluid-particle interaction forces can be improved by using a resolved method [15]. This would account for fluid-particle interactions more realistically at the surface of the particles and boundary surfaces - by using fluid meshes smaller than the particle diameter to apply boundary conditions at the particle surfaces [16]. However, this results in a significant increase in computational intensity. Even with advancing technology, applying these methods in a reasonable amount of time is still not yet practical. Therefore, the current best trade-off between accuracy and computational intensity is the unresolved CFD-DEM approach.

In general, the use of CFD-DEM coupling to simulate solid-liquid systems has been fairly wide spread. Refer to Section 2.6 for the literature review of these applications. However, it should be noted that applications of the CFD-DEM method to solid-liquid mixing systems - in particular - have been quite limited. The few existing CFD-DEM 
simulations of this application have been validated based on a variety of system variables: the just-suspension speed, the particle velocity and displacement trends, the fraction of suspended particles, and the spatial distribution of the particle velocities. However, an extensive analysis of the mixing quality based on the spatial distribution of the particle concentrations is still lacking.

Therefore, the objective of the current study is to comprehensively investigate both the degree of particle suspension and dispersion in a mixing tank operating in the turbulent regime. The previous studies (i.e. having applied ERT or CFD-DEM coupling to solid-liquid mixing) have only covered either the suspension or dispersion of the particles. However, in the current study, we will extensively analyze both quantities. This will be accomplished using mixing indexes. In this regard, a new suspension mixing index will be introduced in this work. Since, those previously used in ERT (i.e. the experimental method also employed in this study) have only quantified the degree of dispersion. Furthermore, a two-way coupled CFD-DEM method will be developed to simulate the mixing system. This will be facilitated by commercial software packages (i.e. coupled EDEM-FLUENT). The accuracy of this model will be evaluated against experimental data obtained using ERT. Then, the model will be used to evaluate the sensitivity of the mixing indexes over a range of model parameters. These include the type of drag force model, the inclusion or exclusion of other fluid-particle interaction forces, and various particle properties. This study will contribute to a larger body of work that will allow CFD-DEM coupling to be used more regularly in the design, optimization, and control of solid-liquid process systems. 
This thesis is structured as follows. Chapter two gives a detailed literature review on the experimental and numerical methods employed in this study. The former involves a review of the ERT method and ERT applications for solid-liquid mixing systems. Whereas, the latter includes an overview of single-phase and two-phase numerical methods as well the CFD-DEM approach and its application to various solid-liquid systems. Chapter three outlines the experimental methodologies undertaken in this study. This includes the experimental set-up design, details on the ERT system and its components, the ERT data analysis approach, and the experimental results. Chapter four describes the CFD-DEM model development used in the current work. Chapter five covers the model evaluation - i.e. comparison between simulation and experimental results - as well as the model sensitivity tests. Finally, chapter six summarizes the general conclusions of the current work and recommendations for future works. 


\section{Chapter 2 : Literature Review}

In this chapter, the experimental measurement technique and numerical modeling approaches used to evaluate solid-liquid systems are described. In particular, the Electrical Resistance Tomography (ERT) measurement technique - used in the current study - is introduced and its application in solid-liquid mixing systems is presented. In terms of numerical methods, various simulation methods will be reviewed. However, the main focus of the discussion is on the coupled Computational Fluid Dynamics (CFD) and Discrete Element Method (DEM) approach. Furthermore, applications of this modeling technique to solid-liquid systems - in general - is presented.

\subsection{Solid-Liquid Mixing}

Solid-liquid mixing is the process of suspending, dispersing, and incorporating of a particle phase within a liquid phase. Depending on the difference in density between 
the solid particles and liquid, the particles can either have a tendency to settle or float. When mixing either of these types of particles, the mixing objective is to optimize the hydrodynamic conditions and prevent the particles from accumulating [17]. In general, this will promote greater mass transfer, heat transfer, or rates of reaction - depending on the types of processes involved. In the majority of cases, solid-liquid mixing is performed in mechanically stirred vessels [18].

Solid-liquid stirred tanks are used in a wide variety of industries. Some common industries include food processing, wastewater treatment, mining and mineral processing, chemical processing, and pharmaceutical manufacturing [19]. This non-exhaustive list illustrates the prevalence and significance of solid-liquid mixing tanks. However, it should be noted that these systems are still not well understood due to the associated complex phenomena [1]. For this reason, a great deal of research has gone into understanding these systems.

The hydrodynamic behavior of a stirred tank can be influenced by a variety of parameters. These include (1) the physical properties of the liquid and solid phases such as the liquid density or particle size; (2) the operating conditions including particle concentration or liquid level; (3) the system dimensions such as impeller clearance or tank diameter; and (4) the agitation conditions such as impeller speed or torque [20]. In literature, the influence of these parameters on the performance of stirred tanks has been evaluated both experimentally and numerically. This evaluation has been facilitated through a variety of measured mixing parameters. These include the just-suspension speed [21]-[24], cloud height [25], power consumption [26], [27], mixing time [28]-[30], and spatial distribution of the particle concentrations [31]-[33]. In the current work, the 
lattermost option was selected in order to perform a more rigorous assessment of the stirred tank performance. In comparison, the spatial distribution of the particle concentrations provides a more detailed and local assessment - when compared to the other parameters, which only provide global assessments of the system.

\subsection{Theoretical Principles of Electrical Resistance Tomography (ERT)}

The spatial distribution of the particle concentrations, in solid-liquid systems, has been measured experimentally with a variety of measurement techniques. These include Magnetic Resonance Imaging (MRI), Positron Emission Particle Tracking (PEPT) and various tomography techniques - x-ray, gamma-ray, optical, ultrasound, and electrical (i.e. either capacitance or resistance) [34]. In general, these methods can be evaluated based on two defining characteristics: (1) the speed in which the images are captured and (2) the accuracy of the captured images. These features are inversely related, in most cases. Such that, higher image resolution comes at the cost of longer image reconstruction times.

In this regard, the Electrical Resistance Tomography (ERT) technique - although relatively fast - has a low image resolution, ranging only from $3-10 \%$ of the vessel diameter [35]. Therefore, the main advantage of this method is its ability to capture highly transient data. Furthermore, this measurement technique has additional advantages associated with simplicity, low cost, and safe operating conditions [36].

Tomography is a method used to determine the spatial distribution of a physical property - across an area - based on the measurements taken at its boundary. In the 
context of ERT, the physical property being determined is the difference in conductivity. Whereas, the quantity being measured at the boundary is the output of voltage produced by an injection of current. The conductivity values are normally converted to particle concentrations to allow the results to be interpreted directly with ease. A basic ERT measurement system is comprised of three components: the sensor interface, the data acquisition system (DAS), and the data processing software.

\subsubsection{Sensor Interface}

Unlike its name suggests, the sensing interface is not only used for detection, it is also used as an excitation source to elicit the responses that it detects. In this regard, the sensing interface alternates between injecting current into the system and measuring the resulting voltage output. This is achieved through a network of cables and electrodes such that the cables connect the electrodes to the DAS and the electrodes are installed in the walls of the tank in an invasive but non-intrusive manner. This allows them to be in direct contact with the secondary liquid without changing the hydrodynamics of the system.

The electrodes can be made of stainless steel, brass, silver, gold, or even platinum [37]. However, the electrode material is generally selected based on the conductivity of the liquid being measured, which should be less conductive than the electrodes [38]. Alternatively, the electrode size is selected based on a variety of variables. The main influences being the size of containing vessel, the operating flow velocity, the required imaging speed, and the conductivity of liquids and solids being measured [39]. However, 
since the electrodes act as both excitation sources and detectors, further considerations are required. In this regard, a compromise on the size of the electrodes is necessary in order to optimize the effectiveness of both current injection and voltage detection. This requires that the electrodes be large enough to inject current evenly [40] and small enough to limit the noise levels of the detected voltages [41].

\subsubsection{Data Acquisition System (DAS)}

The DAS is an electronic system comprised of various components: signal sources, electrode multiplexer arrays, amplifiers, voltmeters, analogue-to-digital converters, signal demodulators, and controllers [42]. This system acts as a bridge between the sensor interface and the data processing software. For this reason, it has a wide variety of functions.

The main function of the DAS is the administration of the measurement process. This involves controlling how measurements are taken, which electrodes are used, and what the electrodes are used to do. However, these actions depend on the data collection strategy being implemented, which can be one of four types of strategies: adjacent, opposite, diagonal, and conducting boundary. In general, the strategy is usually selected based on its ability to distinguish conductivity changes within regions of the system that are of interest. In this regard, the adjacent scheme is more accurate towards the periphery of the tank, while the opposite scheme is more accurate towards the center of the tank, and the diagonal scheme is moderately accurate throughout the entirety of the tank [43]. 
Although, the adjacent scheme is most commonly used because of its minimal hardware requirements and fast image reconstruction speed [3].

\subsubsection{Image Reconstruction}

The data collected by the sensor interface and DAS is relayed to a computer hosting the data processing software. Using this information, the data processing software generates tomographic images that illustrate the spatial distribution of the particle concentrations. The process of generating these images is referred to as image reconstruction - which essentially uses spatial discretization methods to solve for distributed properties in an area from quantities measured at its boundary. In the context of ERT, the voltage readings taken at the electrodes surfaces are used to solve for the conductivity distribution in the sensing planes. This is then converted to a distribution of particle concentrations.

In this regard, the conductivity distribution is determined by discretizing Poisson's equation for electrical resistance and applying boundary conditions for current injection and voltage detection [44]:

$$
\begin{gathered}
\nabla \cdot(\sigma(x, y) \nabla V(x, y))=0 \\
\int \sigma \frac{\partial V}{\partial n}=\left\{\begin{array}{lr}
+I, & \text { Current Source } \\
-I, & \text { Current Sink }
\end{array}\right. \\
V=0 \quad \text { Voltage Reference }
\end{gathered}
$$


where $\sigma$ is the conductivity, $V$ is the voltage, $n$ is a nodal point, and $I$ is the injection current. The resulting system of equations will have the following form:

$$
\underline{Y} \cdot \underline{v}=\underline{c}
$$

where $\underline{\underline{Y}}$ is the conductivity matrix, $\underline{v}$ is the unknown voltage readings; and $\underline{c}$ is the current injection data for the boundaries. This system of equations can be solved either quantitatively with iterative methods or qualitatively with non-iterative methods.

The final step of the image reconstruction process is then converting the conductivity distribution to a distribution of particle concentrations. This can be achieved using Maxwell's equation [39]:

$$
X=\frac{2 \sigma_{\mathrm{liq}}+\sigma_{\mathrm{nc}}-2 \sigma_{m c}-\frac{\sigma_{m c} \sigma_{\mathrm{nc}}}{\sigma_{\mathrm{liq}}}}{\sigma_{m c}-\frac{\sigma_{m c} \sigma_{\mathrm{nc}}}{\sigma_{\text {liq }}}+2\left(\sigma_{\mathrm{liq}}-\sigma_{\mathrm{nc}}\right)}
$$

where $X$ is the volume fraction, $\sigma_{l i q}$ is the conductivity of the continuous phase, $\sigma_{n c}$ is the conductivity of the dispersed phase, and $\sigma_{m c}$ is the measured conductivity.

\subsection{ERT Applications in Solid-Liquid Mixing}

In solid-liquid mixing, the uniformity of the particle concentration distribution is a key parameter used to evaluate the system. It has been studied at length with the ERT measurement technique. This will be the focus of the following discussion. 
McKee et al. [33] studied the uniformity of mixing sieved sand and water in a 30 $\mathrm{cm}$ diameter stirred tank. This was achieved by first evaluating the relative standard deviation values of the particle concentrations at various radial and axial locations. Then, graphing these values to illustrate the degree of particle dispersion (i.e. the uniformity of the particle distribution throughout the stirred tank). In doing so, they found that better dispersion is achieved with higher impeller speeds and lower particles sizes. Williams et al. [45] continued this work and found that the impeller type also influences particle dispersion. However, a primary difference between these studies was that Williams et al. quantified the degree of dispersion into a more convenient value referred to as a mixing index. This parameter was evaluated by averaging the various relative standard deviation values of the particle concentrations at different axial locations. Using a mixing index made it easier to compare multiple datasets. However, their mixing index only accounted for the distribution of the particles in the radial direction.

Alternatively, Hosseini et al. [20] only evaluated the degree of particle dispersion in the axial direction. They did this using a different mixing index referred to as homogeneity. This parameter was evaluated by taking the complement of the relative standard deviation value of the averaged particle concentrations at different axial locations. When increasing the impeller speed, they found that this homogeneity value increased to a maximum value after which it began to decrease. In addition to this, they determined that this maximum value was influenced by a variety of system parameters. These included the impeller type and clearance, particle size, and solid concentration.

In contrast, Stevenson et al. [46] did not use a mixing index or any other standard deviation value to evaluate the mixing quality. Instead, they used the axial and radial 
particle concentration profiles. This allowed them to evaluate not only the degree of particle dispersion but also the degree of particle suspension from the bottom of the tank. Using this approach, they found that the degree of particle dispersion increases in the axial direction with decreasing particle density. While, the degree of particle suspension increases with increasing impeller speed and the rate of increase is influenced by particle size. Such that, the degree of particle suspension increases linearly for smaller particles and exponentially for larger particles. In additional to this, they also confirmed that assumptions of radial uniformity are not always valid. This was also confirmed by Harrison et al. [4] using a different set of mixing index definitions, which quantified the degree of dispersion in not only the radial and axial directions, but also throughout the tank (i.e. overall mixing index). This was facilitated by discretizing the tomographic planes into concentric rings. In doing so, the relative standard deviation of either the planes, annular sections, or entire tank could be evaluated. These values were then used to determine the different types of mixing indexes. By comparing these mixing indexes, they determined that particle dispersion is dominated by radial distributions for fine particles and axial distributions for medium to large particles. Depending on the particle size, one might neglect the axial or radial distributions based on this finding. However, Harrison et al. [4] still recommends that both distributions be taken into consideration in order to improve the accuracy of quantifying the mixing quality. Using their overall mixing index, they confirmed that the degree of dispersion increases with increasing solids concentration and decreasing particle size. They also found that increasing the impeller speed decreased the overall mixing index to a minimum value after which it remained relatively constant. 
Carletti et al. [47] used a different overall mixing index based on the relative standard deviation of the square tomographic elements throughout the tank. This definition allowed them to apply the same principles as Harrison et al. [4] to account for both the radial and axial particle distributions. However, they had the added advantage of not being limited to a certain tomographic element shape. By comparing their definition with those proposed by Williams et al. [45] and Hosseini et al. [20], they also confirmed that the radial distribution was more significant for fine particles. Mishra and Ein-Mozaffari [48] also compared various mixing index definitions. Specifically, those proposed by Williams et al. [45], Hosseini et al. [20], and Carletti et al. [47]. In doing so, they confirmed that the axial distribution was dominant for the large particles used in their study. For this reason, they opted to only use the axial mixing index proposed by Hosseini et al. [20] to further evaluate their results. In doing so, they confirmed that the homogeneity increased, with increasing impeller speed, to a maximum value after which it began to decrease. They also found that the maximum homogeneity value was influenced by the presence of baffles. While, confirming that it was also influenced by the impeller type and clearance, particle size, and solid concentration.

\subsection{Numerical Simulation Methods}

This section covers the numerical approaches used to simulate two-phase systems. However, the main focus is CFD-DEM coupling. To gain a better understanding of this method, the fundamentals and formulations of both CFD and DEM approaches used to model single-phase systems - are first introduced. Then, the coupled CFD-DEM 
method is introduced. The advantages and disadvantages of this method are then highlighted in comparison with alternative two-phase models.

\subsubsection{Single-Phase Modeling}

Single phases can be represented by discrete or continuum based descriptions. Such that, the former requires a Lagrangian type of model and the latter requires an Eulerian type of model [13], [16]. Lagrangian and Eulerian models are discussed in following sub-sections.

\subsubsection{Lagrangian Models - Discrete Element Method (DEM)}

Lagrangian models are conventionally used to model solid particle motion. However, they also can be used at the microscopic level to model fluid motion [49]. When modeling fluid motion, these methods can be very computationally intensive due to the great number of particles involved. For the sake of brevity, these types of models will not be discussed any further. One of the most common Lagrangian models used to simulate particle motion is referred to as the Discrete Element Method (DEM). This modeling approach is used to study the dynamic behaviour in systems comprised of distinct elements - i.e. particles. DEM provides particle-scale information such as particle positions and velocities, as well as the forces acting on the particles. In the DEM approach, particle collisions are described with two distinct models: hard-sphere and soft- 
sphere. The main difference between these models is the assumptions used to handle collisions.

The hard-sphere model assumes that particles are rigid and that collisions occur over a very short period of time. As a result, collisions are considered to be instantaneous and are assumed to only occur one at a time [50]. This is a realistic assumption for a dilute system in which collisions are infrequent and short. In this model, the particle velocities and positions are updated at each collision - also referred to as an event [51]. For this reason, this method is described as event-driven. During each collision, the particles' post-collision velocities are calculated based on their pre-collision velocities. Although, it should be noted that the hard-sphere model cannot be applied to dense systems - it is limited only to dilute systems [52], [53]. Alternatively, the soft-sphere model can be applied to both dilute and dense systems.

The soft-sphere model assumes that particles are elastic and that collisions are gradual. As a result, multiple collisions can occur at the same time. This is taken into consideration by using a small and fixed time step to monitor the system and detect all of the collisions. This method is referred to as time-driven [13]. During each time step, the particles' subsequent velocities are determined by numerically integrating Newton's second law of motion, which is as follows [54]:

$$
m_{i} \frac{d \boldsymbol{v}_{\boldsymbol{i}}}{d t}=\sum_{j}^{n} \boldsymbol{F}_{\boldsymbol{i j}} \quad, \quad I_{p, i} \frac{d \boldsymbol{\omega}_{\boldsymbol{i}}}{d t}=\sum_{j}^{n} \boldsymbol{M}_{\boldsymbol{i j}}
$$

where $i$ represents particle $i, j$ represents particle j, $m$ is the mass, $I_{p}$ is the moment of inertia, $\boldsymbol{v}$ is the translational velocity, $\boldsymbol{\omega}$ is the angular velocity, $\sum \boldsymbol{F}_{\boldsymbol{i}}$ is the total forces 
acting on a particle, and $\sum \boldsymbol{M}_{\boldsymbol{i}}$ is the total torques acting on a particle. The force term includes not only the contacting forces, but also non-contacting, gravitational, and external forces. The non-contacting forces can include Van der Waals, electrostatic, and capillary forces [14].

The contacting forces and torques are determined using constitutive models. These contact models relate the contact forces and torques to the degree of particle overlap with other contacting particles or walls. The normal overlap $\left(\delta_{n}\right)$ and tangential overlap $\left(\delta_{t}\right)$ of particle-particle and particle-wall contacts are illustrated in Figure 1. In this figure, $\boldsymbol{R}_{\boldsymbol{c}}$ is the normal distance from the center of the particle to the contact plane, $C$ is the contact point, and $\boldsymbol{g}$ is the acceleration due to gravity.
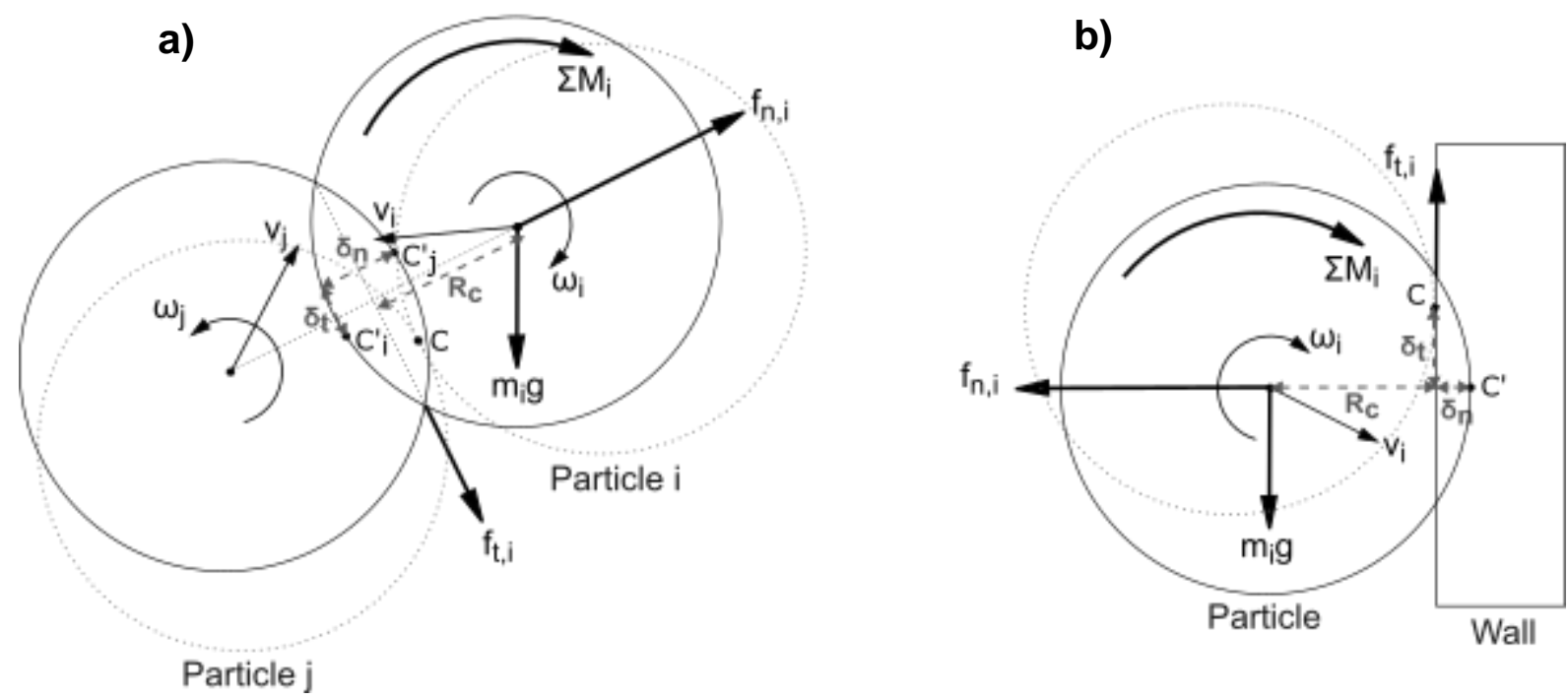

Figure 1. Contact diagrams for a) particle-particle and b) particle-wall contacts.

There are a variety of contact models that can be used. In this regard, they can account for elastic, dissipative, frictional, or a combination of these type of interactions 
[55]. Some commonly used contact models are as follows: the linear spring-dashpot model proposed by Cundall and Strack [54], the model proposed by Langston et al. [56], and simplified versions of the Hertz-Mindlin-Deresiewicz model. The Hertz-MindlinDeresiewicz model accounts for complex interactions in a realistic manner. This is achieved by combining the theory developed by Hertz [57] for the normal contact forces and the theory developed by Mindlin and Deresiewicz [58] for the tangential forces. However, this method can be quite computationally intensive for larger system [14]. For this reason, simplified variations of this model are more commonly used in literature. Examples of such models include those proposed by Tsuji et al. [59], Walton and Braun [60], and Thornton and Yin [61].

When implementing the soft-sphere model, the motion of particles is estimated through iterative time integration of Newton's second law of motion. There are several different time integration schemes that have been used in literature. Some commonly used methods include the Euler, position Verlet, and velocity Verlet methods [62]. When implementing this method, the time step must be sufficiently small in order to produce a stable numerical solution. In general, the DEM time step is selected based on the critical time step at which simulations would become unstable. Such that, the time step is selected to be smaller than the critical time step. There are few methods that can be used to determine the critical time step. In one of the most common methods used in literature, the critical time step is equivalent to the Rayleigh time step. This is the time required for a shear wave to propagate through an elastic body and can be represented as follows [63]: 


$$
\Delta t_{\text {Ray }}=\frac{\pi R_{\min }}{0.1634 v+0.8766} \sqrt{\frac{\rho_{p}}{G_{\max }}}
$$

where $R_{\text {min }}$ is the minimum particle radius, $\rho_{p}$ is the particle density, $v$ is the Poisson ratio, and $G_{\max }$ is the maximum shear modulus. Another common method used to calculate the critical time step is as follows [59]:

$$
\Delta t_{c r}=\frac{\pi}{5} \sqrt{\frac{m}{k}}
$$

where $\Delta t_{c r}$ is the critical time step, and $k$ is the spring constant. This approach is based on the time period required for free oscillation of the system [55]. Alternatively, O'Sullivan and Bray [64] determined the critical time step through a stiffness analysis of the total system energy balance. Although, it should be noted that this method is more numerically complex than those previously discussed.

\subsubsection{Eulerian Models - Computational Fluid Dynamics (CFD)}

Eulerian models are conventionally used to model fluid motion. In this regard, the following two Eulerian models are commonly used: the Lattice-Boltzmann (LB) Model and Computational Fluid Dynamics (CFD) [16]. The LB model was derived within a statistical mechanics framework. In this model, the fluid motion is governed by the Boltzmann equation, which provides a microscopic description of the fluid [65]. Alternatively, the CFD governing equations were derived within a continuum mechanics framework. This method 
is the most commonly used model for simulating fluid motion. The conservation of mass and momentum equations used in the CFD method are as follows [66]:

$$
\frac{\partial \rho_{f}}{\partial t}+\nabla \cdot\left(\rho_{f} \boldsymbol{u}\right)=0 \quad, \quad \frac{\partial\left(\rho_{f} \boldsymbol{u}\right)}{\partial t}+\nabla \cdot\left(\rho_{f} \boldsymbol{u u}\right)=-\nabla p-\nabla \cdot \boldsymbol{\tau}-\rho_{f} \boldsymbol{g}
$$

where $\boldsymbol{u}$ is the local fluid velocity, $\rho_{f}$ is the fluid density, $p$ is pressure, and $\boldsymbol{\tau}$ is the stress tensor. The momentum equations are commonly referred to as the Navier-Stokes equations.

Both of these methods have been proven to be capable of modeling fluid motion. However, they also both have distinctive strengths and weaknesses. In this regard, the LB model is generally more memory and CPU intensive than the CFD method [67]. However, there are certain circumstances in which the LB model can become more efficient than the CFD method. This is the case for complex geometries, such as porous media [68]. However, the LB approach does not fall within the scope of the current work and therefore will not be discussed any further. Refer to [67] for more information on the LB method. The following discussion will now focus solely on the CFD approach.

Prior to running any CFD simulations, the geometry is discretized into a mesh consisting of interconnecting elements. This is referred to as mesh generation. The shape of the mesh elements will depend on the type of discretization scheme selected. In this regard, a variety of discretization schemes can be used such as the finite-element, finitedifference, and finite-volume methods [13]. For 3D geometries, the meshes can be comprised of tetrahedra (i.e. prism or pyramid) or hexahedra elements. It should be noted that the element shape and dimensions can be varied throughout the geometry. This is 
advantageous for two reasons. First, it can reduce computations costs since the total number of mesh elements in the geometry can be optimized. Second, the model accuracy can be improved by having refined elements near the geometry walls [69]. The mesh quality has a significant influence on the simulation convergence, solution accuracy, and CPU time requirements. For this reason, extra precaution should be taken to ensure good mesh quality during the mesh generation phase.

By discretizing the geometry into a mesh, the governing equations of the CFD method - as shown in Equation ( 9 ) - can be approximated with a set of algebraic equations. To solve this set of equations, a numerical scheme is required for coupling the pressure and momentum quantities. Some of the common velocity-pressure coupling schemes include the Semi-Implicit Method for Pressure-Linkage Equations (SIMPLE) [70], SIMPLE-Consistent (SIMPLEC) [71], and the Pressure Implicit with Splitting of Operators (PISO) scheme [72]. Moreover, the discretization of the governing equation parameters is also required. This is commonly achieved with numerical methods such as the first-order upwind, second-order upwind, power law, and QUICK schemes. Lastly, temporal discretization is also necessary. Popular methods used for this purpose include first-order or second-order implicit schemes [73].

When operating within the turbulent regime, turbulence closure equations must also be incorporated within the CFD model. Some commonly used turbulence models are

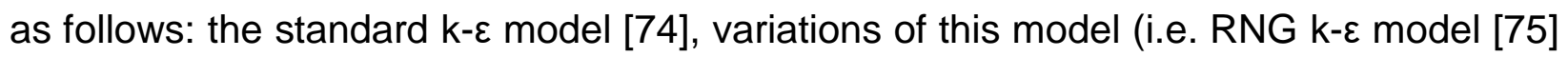

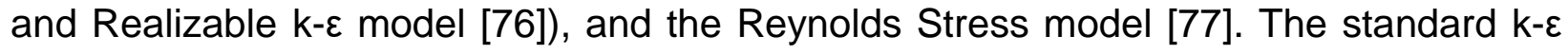
model is based on the concept of eddy viscosity and the assumption of isotropic stresses. It is reasonably accurate for a wide variety of turbulent flows. However, it has been found 
to be less reliable in complex flows that have strong streamline curvature, vortices, and swirling flow. To mitigate this issue, modified versions of the $k-\varepsilon$ model have been used in literature [73]. Alternatively, the Reynolds Stress model can also be used to improve the accuracy of modeling complex flows. Since, this model accounts for anisotropic stresses and solves for the entire Reynolds stress tensor. Although, it should be noted that this model is significantly more computationally intensive than those previously discussed [78].

\subsubsection{Two-Phase Modeling}

When modeling two-phase systems, either phase can be represented by a Lagrangian or Eulerian description. In this regard, fluid-particle systems are simulated with models that fall under the following categories: Lagrangian-Lagrangian, EulerianLagrangian, and Eulerian-Eulerian [79]. In general, these modeling approaches can be evaluated based on two defining characteristics: (1) their computational efficiency and (2) the accuracy in which they capture fluid and particle physics. In most models, these features are inversely related - such that greater accuracy comes with a lower computational efficiency.

The Lagrangian-Lagrangian approach is very reliable in terms of capturing both fluid and particle physics. However, this comes at a very high computational cost. This is due to the fact that the fluid and particle phases will both be represented by discrete elements. For this reason, this approach is generally restricted to modeling very small systems - at least with an average hardware setup [16]. 
In contrast, Eulerian-Eulerian models are the least computationally intensive and can be used to simulate a variety of system sizes - ranging from small systems to larger and more practical geometries [16]. This type of model is also referred to as a Two Fluid Model (TFM) [80]. In this modeling approach, both the fluid and particle phases are represented as continua. Such that, models are used to approximate the effects of particle-particle and fluid-particle interactions. However, with the current state of these equations, this normally also results in a loss of accuracy [13]. Furthermore, it should also be noted that particle-scale phenomena cannot be captured with this modeling approach.

In contrast to TFM, the Eulerian-Lagrangian approach represents the fluid phase as a continuum and the particle phase as discrete elements. This more accurately captures particle physics as compared to TFM [14]. This modeling approach is divided into two broad categories that depend on the size of the mesh - used to model the fluid phase. In this regard, the fluid mesh can either be an order of magnitude smaller or larger than the particle size. The former is referred to as a resolved model and the latter is referred to as an unresolved model. The resolved Eulerian-Lagrangian models are more accurate at capturing fluid-particle physics. However, this comes at a significantly higher computational cost. Some examples of resolved Eulerian-Lagrangian models are as follows: the Lattice-Boltzmann model coupled with DEM (LB-DEM) [81], and Direct Numerical Simulation coupled with DEM (DNS-DEM) [82], the Distributed Lagrange Multiplier (DLM) method [83], and PHYSALIS [84]. In comparison, the unresolved Eulerian-Lagrangian approach is not as accurate in capturing fluid-particle physics - as compared to the resolved methods. However, it does have a more manageable computational intensity. Therefore, this approach offers the best trade-off between 
accuracy and computational efficiency - at least with current technological capabilities [14]. The most common unresolved Eulerian-Lagrangian model used in literature is CFDDEM coupling. This method will be discussed in further detail in Section 2.5.

\subsection{Theoretical Principles of Computation Fluid Dynamics (CFD) and Discrete Element Method (DEM) Coupling Model}

As previously mentioned, CFD-DEM coupling is an unresolved EulerianLagrangian method that combines the CFD and DEM methodologies. In this approach, the motion of the fluid phase is simulated by locally-averaged Navier-Stokes equations [85]. The interactions between the fluid and solid particles are accounted for with equivalent momentum exchange terms in the locally-averaged Navier-Stokes momentum equation governing fluid motion and the Newtonian equation governing particle motion [53]. Such that, various models are used to estimate the fluid-particle interaction forces [16]. These aspects of the CFD-DEM model will be discussed in the following subsections. In addition to this, other topics covered include potential coupling schemes, further simulation considerations, available software options, and a summary of the advantages and disadvantages of CFD-DEM coupling.

\subsubsection{General Overview of Governing Equation Forms}

The governing equations for the CFD-DEM method have had two distinctive configurations in literature: Model A and Model B. In general, the main difference between 
these configurations is the pressure distribution assumption used to derive the governing equations.

Model $\mathrm{A}$ assumes that pressure is distributed amongst both phases and can be represented by the following equations [59], [86]:

$$
\begin{gathered}
\frac{\partial \varepsilon_{f} \rho_{f}}{\partial t}+\nabla \cdot\left(\varepsilon_{f} \rho_{f} \boldsymbol{u}\right)=0 \quad, \quad \frac{\partial\left(\varepsilon_{f} \rho_{f} \boldsymbol{u}\right)}{\partial t}+\nabla \cdot\left(\varepsilon_{f} \rho_{f} \boldsymbol{u} \boldsymbol{u}\right)=-\varepsilon_{f} \nabla p-\boldsymbol{F}_{\boldsymbol{p f}}+\nabla \cdot\left(\varepsilon_{f} \boldsymbol{\tau}\right)+\varepsilon_{f} \rho_{f} \boldsymbol{g} \\
m_{i} \frac{d \boldsymbol{v}_{\boldsymbol{i}}}{d t}=\sum_{j}^{n} \boldsymbol{f}_{\boldsymbol{i j}}^{\boldsymbol{c}}+m_{i} \boldsymbol{g}+\boldsymbol{f}_{\boldsymbol{p} \boldsymbol{f}, \boldsymbol{i}} \quad, \quad I_{p, i} \frac{d \boldsymbol{\omega}_{\boldsymbol{i}}}{d t}=\sum_{j}^{n} \boldsymbol{M}_{\boldsymbol{i j}} \\
\boldsymbol{F}_{\boldsymbol{p} \boldsymbol{f}}=\frac{\sum\left(\boldsymbol{f}_{\boldsymbol{d}, \boldsymbol{i}}+\boldsymbol{f}^{\prime \prime}\right)}{\Delta V} \quad, \quad \boldsymbol{f}_{\boldsymbol{p} \boldsymbol{f}, \boldsymbol{i}}=\boldsymbol{f}_{\boldsymbol{d}, \boldsymbol{i}}+\boldsymbol{f}_{\nabla \boldsymbol{p}, \boldsymbol{i}}+\boldsymbol{f}^{\prime \prime}
\end{gathered}
$$

where $\varepsilon_{f}$ is the local fluid porosity, $\boldsymbol{F}_{\boldsymbol{p} \boldsymbol{f}}$ is the volumetric fluid-particle interaction force, $\Sigma \boldsymbol{f}_{i j}^{c}$ is the total contact force, $\boldsymbol{f}_{p f}$ is the individual overall fluid-particle interaction force, and $\Sigma \boldsymbol{M}_{\boldsymbol{i j}}$ is the total contact torque, $\boldsymbol{f}_{\boldsymbol{d}}$ is the drag force, $\boldsymbol{f}_{\nabla \boldsymbol{p}}$ is the pressure gradient force, $\boldsymbol{f}^{\prime \prime}$ is the sum of all other fluid-particle interaction forces acting on particle $i$, and $\Delta V$ is the mesh cell volume for the fluid phase.

Alternatively, Model B assumes that pressure is distributed only within the fluid phase. This results in a different set of equations for the momentum conservation of the fluid phase and the fluid-particle interaction forces. These equations are as follows [87]:

$$
\begin{gathered}
\frac{\partial\left(\varepsilon_{f} \rho_{f} \boldsymbol{u}\right)}{\partial t}+\nabla \cdot\left(\varepsilon_{f} \rho_{f} \boldsymbol{u u}\right)=-\nabla p-\boldsymbol{F}_{\boldsymbol{p} \boldsymbol{f}}+\nabla \cdot\left(\varepsilon_{f} \boldsymbol{\tau}\right)+\varepsilon_{f} \rho_{f} \boldsymbol{g} \\
\boldsymbol{F}_{\boldsymbol{p} \boldsymbol{f}}=\frac{\sum \boldsymbol{f}_{\boldsymbol{p} \boldsymbol{f}, \boldsymbol{i}}}{\Delta V} \quad, \quad \boldsymbol{f}_{\boldsymbol{p} \boldsymbol{f}, \boldsymbol{i}}=\boldsymbol{f}_{\boldsymbol{d}, \boldsymbol{i}}+\boldsymbol{f}_{\boldsymbol{\nabla} \boldsymbol{p}, \boldsymbol{i}}+\boldsymbol{f}^{\prime \prime}
\end{gathered}
$$


Furthermore, Model B has also been found in a simplified form in literature - under an assumption of steady and uniform fluid flow. This results in a different equation for the fluid-particle interactions forces, which is as follows:

$$
\boldsymbol{F}_{\boldsymbol{p} \boldsymbol{f}}=\frac{\sum\left(\boldsymbol{f}_{\boldsymbol{d}, \boldsymbol{i}}+\boldsymbol{f}^{\prime \prime}\right)}{\varepsilon_{f} \Delta V}-\frac{\sum\left(\rho_{f} V_{p, i} \boldsymbol{g}\right)}{\Delta V} \quad, \quad \boldsymbol{f}_{\boldsymbol{p} \boldsymbol{f}, \boldsymbol{i}}=\frac{\left(\boldsymbol{f}_{\boldsymbol{d}, \boldsymbol{i}}+\boldsymbol{f}^{\prime \prime}\right)}{\varepsilon_{f}}-\rho_{f} V_{p, i} \boldsymbol{g}
$$

where $V_{p, i}$ is the volume of particle $i$.

It has been argued by Zhou et al. [88] that Model A may be ill-posed and Model B is more logical and acceptable. Both models were evaluated by the authors - in their original forms - and were found to produce comparable and logical simulation results. However, this was not the case for the simplified version of Model B. This model was found to be unreliable, when applied to an unsteady and non-uniform fluid flow - i.e. within a hydrocyclone. For this reason, the authors recommended that Model A and Model B should only be used in their original forms in future works. Refer to [88] for a more comprehensive discussion on this topic.

\subsubsection{Modeling of Fluid-Particle Interaction Forces}

The fluid-particle interaction terms - included in Equations ( 12 ), ( 14 ) and ( 15 ) - can account for a variety of forces. Potential forces that can be included are listed and defined in Table 1. Including all these forces in the model can become quite computationally taxing. For this reason, insignificant forces are usually neglected to decrease computational costs and reduce additional sources of error. 


\section{Table 1. Fluid-particle interaction forces definitions.}

Force

Definition

Citation

$$
\begin{gathered}
\text { Pressure } \quad \text { Accounts for difference in pressure due to buoyancy of } \\
\text { Gradient } \\
\text { particles and acceleration of fluid }
\end{gathered}
$$

\begin{tabular}{|c|c|c|}
\hline $\begin{array}{l}\text { Virtual } \\
\text { Mass }\end{array}$ & Required to displace fluid around accelerating particles & [89] \\
\hline Basset & $\begin{array}{c}\text { Accounts for lagging development of the viscous boundary } \\
\text { layer around accelerating particles }\end{array}$ & [90] \\
\hline Saffman & $\begin{array}{l}\text { Accounts for difference in pressure due to shear flows acting } \\
\text { on non-rotating particles }\end{array}$ & [91] \\
\hline Magnus & $\begin{array}{l}\text { Accounts for difference in pressure due to rotation of particles } \\
\qquad \text { in uniform flows }\end{array}$ & [92] \\
\hline Lubrication & $\begin{array}{l}\text { Hinders particles, in close proximity, from moving towards or } \\
\text { away from each other due to presence of interstitial fluid }\end{array}$ & [93] \\
\hline
\end{tabular}

In the majority of cases, the drag force is significant and dominant. Therefore, it is always taken into consideration. This force can be represented generally in the following form:

$$
\boldsymbol{f}_{\boldsymbol{d}}=\beta_{p f}(\boldsymbol{u}-\boldsymbol{v})
$$

such that $\beta_{p f}$ depends on the specific drag model. There are numerous equations to represent this term. Refer to Table 2 for the different forms of this coefficient used in the Di Felice, Gidaspow, and Syamlal O'Brien drag models. Such that, $d_{p}$ is the particle 
diameter, and $\mu_{f}$ is the fluid viscosity. These models are commonly used and recommended for dense particle suspensions.

Table 2. Drag model coefficients.

\begin{tabular}{|c|c|c|c|}
\hline Model & Equation & & Citation \\
\hline Di Felice & $\begin{array}{c}\beta_{p f}=\frac{3}{4} \frac{\rho_{f}\left(1-\varepsilon_{f}\right) \varepsilon_{f}}{d_{p}} \varepsilon_{f}^{1-\chi} C_{D(D i F)}|\boldsymbol{u}-\boldsymbol{v}| \\
\chi=3.7-0.65 \exp \left[-\frac{\left(1.5-\log _{10} R e_{p}\right)^{2}}{2}\right] \\
C_{D(D i F)}=\left(0.63+\frac{4.8}{R e_{p}^{0.5}}\right)^{2} \\
R e_{p}=\frac{\varepsilon_{f} \rho_{f} d_{p}|\boldsymbol{u}-\boldsymbol{v}|}{\mu_{f}}\end{array}$ & $(17)$ & [94] \\
\hline Gidaspow & $\begin{array}{c}\beta_{p f}= \begin{cases}150 \frac{\left(1-\varepsilon_{f}\right)^{2}}{\varepsilon_{f}} \frac{\mu_{f}}{d_{p}^{2}}+1.75\left(1-\varepsilon_{f}\right) \frac{\rho_{f}}{d_{p}}|\boldsymbol{u}-\boldsymbol{v}|, & \varepsilon_{f}<0.8 \\
\frac{3}{4} \frac{\rho_{f}\left(1-\varepsilon_{f}\right) \varepsilon_{f}}{d_{p}} \varepsilon_{f}^{-2.65} C_{D(\text { Gid })}|\boldsymbol{u}-\boldsymbol{v}|, & \varepsilon_{f} \geq 0.8\end{cases} \\
C_{D(\text { Gid })}= \begin{cases}\frac{24}{R e_{p}}\left[1+0.15 R e_{p}^{0.687}\right], & R e_{p}<1000 \\
0.44, & R e_{p} \geq 1000\end{cases} \end{array}$ & $(18)$ & [87] \\
\hline O'Brien & $\begin{array}{c}\beta_{p f}=\frac{3}{4} \frac{\rho_{f}\left(1-\varepsilon_{f}\right) \varepsilon_{f}}{d_{p}} C_{D(\text { Sym })}|\boldsymbol{u}-\boldsymbol{v}| \\
C_{D(\text { Sym })}=\frac{1}{v_{r s}^{2}}\left[0.63+\frac{4.8}{\left(\operatorname{Re} / v_{r s}\right)^{0.5}}\right]^{2} \\
v_{r s}=\frac{1}{2}\left\{\mathrm{~A}-0.06 \operatorname{Re}+\left[(0.06 \mathrm{Re})^{2}+0.12 \operatorname{Re}(2 \mathrm{~B}-\mathrm{A})+\mathrm{A}^{2}\right]^{0.5}\right\} \\
\mathrm{A}=\varepsilon_{\mathrm{f}}^{4.14} \\
\mathrm{~B}= \begin{cases}0.8 \varepsilon_{\mathrm{f}}^{1.28}, & \varepsilon_{\mathrm{f}} \leq 0.85 \\
\varepsilon_{\mathrm{f}}^{2.65} & \varepsilon_{\mathrm{f}}>0.85 \\
R e=\frac{\rho_{f} d_{p}|\boldsymbol{u}-\boldsymbol{v}|}{\mu_{f}}\end{cases} \end{array}$ & ( 19 ) & [95] \\
\hline
\end{tabular}

The significance of the other fluid-particle interaction forces, however, can vary from case to case. This depends on the operating parameters. For instance, the pressure 
gradient, virtual mass, and Basset forces are often insignificant at high particle-fluid density ratios (i.e. $\rho_{p} / \rho_{f} \geq 1000$ ). However, they can become more significant with lower particle-fluid density ratios that are closer to one [96]. This is due to the unsteady nature of the forces. Alternatively, the Saffman lift force is more significant in high-shear applications. Whereas, the Magnus lift force is more significant in applications with high rates of particle-particle and particle-wall collisions, which cause greater particle rotation [51]. The lubrication force, on the other hand, is generally more pronounced in solid-liquid systems as opposed to gas-solid systems [93]. Refer to Table 3 for the equations used to account for these forces - with the exception of the lubrication force. The lubrication force is normally accounted for implicitly, through the modification of the particle contact force model or coefficient of restitution [97].

Table 3. Other fluid-particle interaction force models.

\begin{tabular}{|c|c|c|c|}
\hline Force & Equation & & Citation \\
\hline $\begin{array}{l}\text { Pressure } \\
\text { Gradient }\end{array}$ & $\boldsymbol{f}_{\nabla \boldsymbol{p}}=-\frac{\pi}{6} d_{p}^{3} \rho_{f}\left(\boldsymbol{g}+\boldsymbol{u} \frac{d \boldsymbol{u}}{d x}\right)$ & $(20)$ & [85] \\
\hline $\begin{array}{l}\text { Virtual } \\
\text { Mass }\end{array}$ & $\begin{array}{c}\boldsymbol{f}_{\boldsymbol{v} \boldsymbol{m}}=\frac{\pi}{12}\left[2.1-\frac{0.132}{0.12+A_{c}^{2}}\right] d_{p}^{3} \rho_{f}\left(\frac{d \boldsymbol{u}}{d t}-\frac{d \boldsymbol{v}}{d t}\right) \\
A_{C}=\frac{(\boldsymbol{u}-\boldsymbol{v})^{2}}{d_{p}} \frac{d(\boldsymbol{u}-\boldsymbol{v})}{d t}\end{array}$ & (21) & [89] \\
\hline Basset & $\begin{array}{c}\boldsymbol{f}_{\text {Bass }}=C_{\text {Bass }}\left[\int_{0}^{t} g\left(t-t^{\prime}\right)\left(\frac{\partial \boldsymbol{H}}{\partial t^{\prime}}\right) d t^{\prime}-g(t) \boldsymbol{H}(t=0)\right. \\
C_{\text {Bass }}=\frac{3}{2} d_{p}^{2} \sqrt{\pi \rho_{f} \mu_{f}} \\
g(t)=\frac{1}{\sqrt{t}} \\
\boldsymbol{H}=\boldsymbol{u}-\boldsymbol{v}+\frac{1}{24} d_{p}^{2} \nabla^{2} \boldsymbol{u}\end{array}$ & ( 22 ) & [90] \\
\hline
\end{tabular}




\begin{tabular}{|cc|}
\hline $\begin{array}{c}\text { Saffman } \\
\text { Lift }\end{array}$ & $\boldsymbol{f}_{\text {Saff }}=1.61 d_{p}^{2}\left(\mu_{f} \rho_{f}\right)^{0.5}\left|\boldsymbol{\omega}_{\boldsymbol{c}}\right|^{-0.5}\left[(\boldsymbol{u}-\boldsymbol{v}) \times \boldsymbol{\omega}_{\boldsymbol{c}}\right]$ \\
& $\boldsymbol{\omega}_{\boldsymbol{c}}=\nabla \times \boldsymbol{u}$
\end{tabular}

\subsubsection{Coupling Approaches and Schemes}

Interphase coupling has three distinctive approaches in literature: one-way, twoway, and four-way coupling. The selection of the approach depends on the system particle concentration, which influences the significance of particle-particle and particleto-fluid interactions [99]. The regime map based on the particle concentration is illustrated in Figure 2.

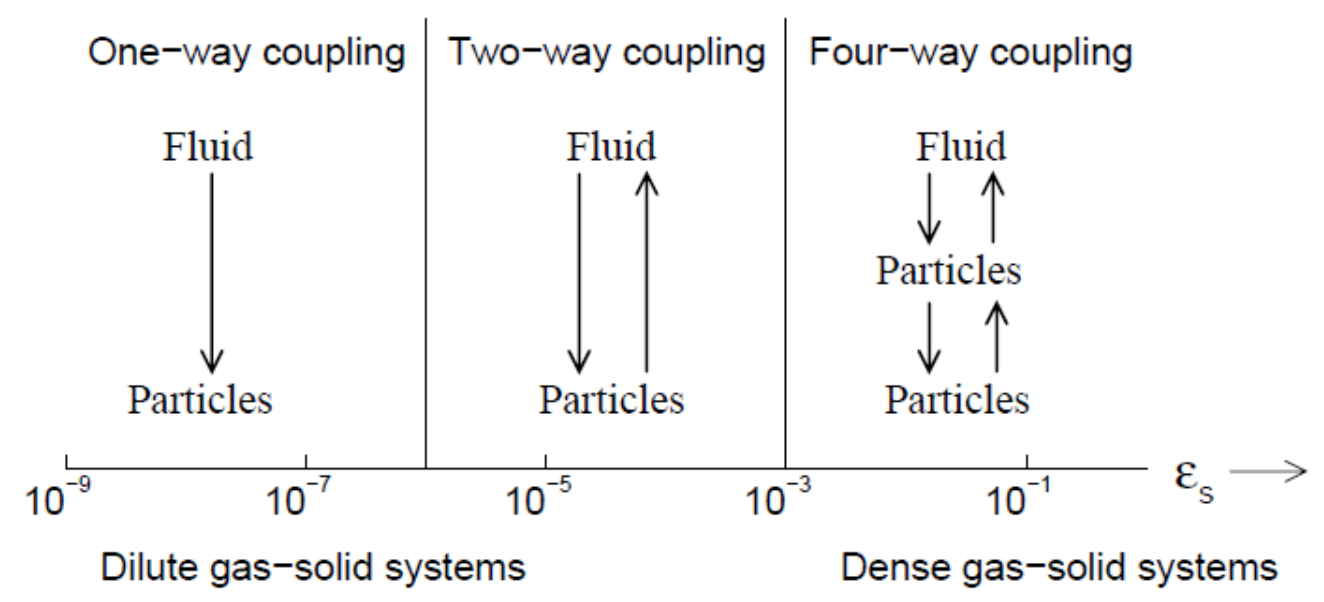

Figure 2. Interphase coupling regime map [13]. 
As seen in this figure, one-way and two-way coupling are used for the simulation of dilute concentration systems and four-way coupling is used for the simulation of dense concentration systems. In the one-way coupling regime, the concentration of particles is so dilute that the particles will likely not interact with each other. Rather, the particles will only interact with the fluid and the fluid will not be significantly influenced by the particles. For these reasons, particle-particle and particle-to-fluid interactions are neglected. In the two-way coupling regime, the concentration of particles is less dilute such that the particles will now influence the fluid but still not be likely to interact with each other. Therefore, only particle-particle interactions are neglected, when simulating a system in this regime. In the four-way coupling regime, the concentration of particles is considered to be dense. In such conditions, the particles will not only interact with the fluid, but also with each other. For this reason, all interactions are taken into consideration [13], [99].

Within the four-way coupled approach, three distinctive coupling schemes have been used in literature - to transfer fluid and particle data between the CFD and DEM solvers to calculate the fluid-particle interaction forces. Zhou et al. [88] reviewed these schemes and recommended that only one should be used in future works. It should be noted that this is also the scheme used in the majority of current CFD-DEM applications. For these reasons, only this scheme will be taken into consideration in the following discussion. Refer to [14], [88] for details on the alternative coupling schemes and their specific limitations.

The calculation cycle and coupling process associated with the recommended coupling scheme is illustrated in Figure 3. The particle positions and velocities are used to calculate the distribution of the fluid porosity term and the volumetric fluid-particle 
interaction forces. In this regard, the individual fluid-particle interaction forces - calculated based on the individual particle velocities - are volume averaged to produce the volumetric fluid-particle interaction force acting on the fluid phase. Next, the CFD solver uses the fluid porosity distribution and volumetric fluid-particle interaction forces to update the fluid velocity field. This information is then used to update the individual fluid-particle interaction forces that will be used in the DEM solver. As the next step, the DEM solver updates the particle positions and velocities, which will then be used to repeat the calculation cycle.

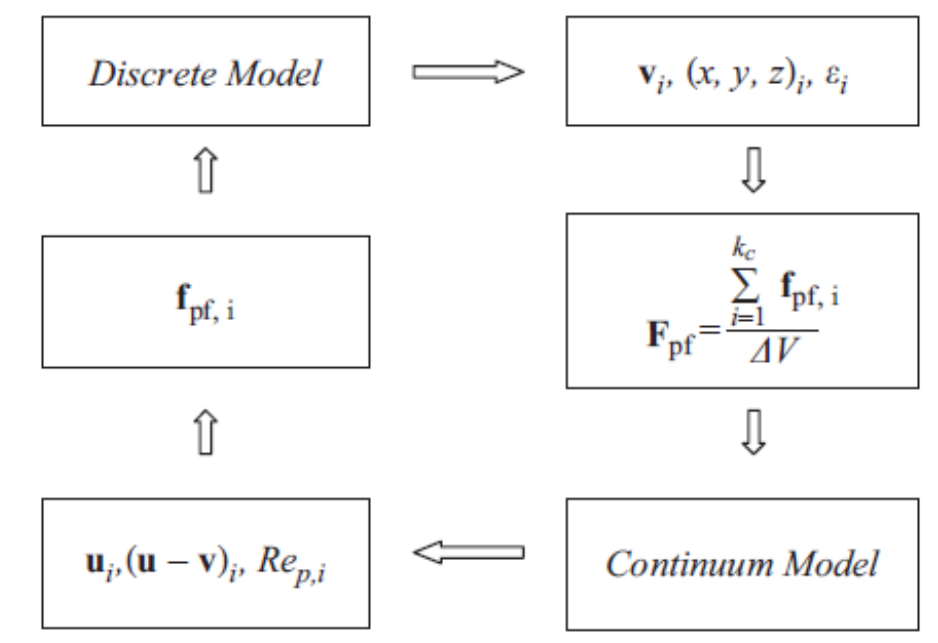

Figure 3. Calculation cycle for coupling scheme [14].

\subsubsection{Mesh Size and Time Step Restrictions}

When using the CFD-DEM method, the mesh size must be large compared to the particle diameter but still small compared to the computational domain [85]. It was recommended by Itasca [100] that the mesh size to particle diameter ratio should be no 
less than 5; and the system width to mesh size ratio should also be no less than 5 . This is represented by the following equations:

$$
\frac{\Delta x_{C F D}}{d_{p}}>5 \quad, \quad \frac{\mathrm{W}}{\Delta x_{C F D}}>5
$$

where $\Delta x_{C F D}$ is the mesh size, and $W$ is the system width. Although, it should be noted that it can be difficult to maintain a mesh size that is small in comparison to the computational domain, under this condition, for narrow or complex geometries. This is an inherent limitation of CFD-DEM coupling. In this regard, grid independence tests should always be performed to verify the accuracy associated with the mesh size.

Additionally, when applying the CFD-DEM method, restrictions are also placed on the size of the CFD time step. In this regard, the CFD time step should be selected based on the DEM time step. It is recommended that the ratio between these time steps - i.e. the CFD time step divided by the DEM time step - be greater than 10 but less than 1000 [101]. Although, typical values found in solid-liquid systems have ranged from 10 to 100 [92], [102]-[109].

\subsubsection{Simulation Software}

Even though there are all-in-one programs designed specifically for executing CFD-DEM coupling, the combination of CFD software with DEM software is more commonly used. This approach is more advantageous as it facilitates independent code 
development [110]. The CFD and DEM software used in literature can be categorized into the following three groups: commercial, open-source, and in-house.

Commercial software tends to be more user friendly and require only a modest amount of computer experience. This is further complemented by the support available from the software developers, regular maintenance, and accelerated software development. For these reasons, commercial software is quite popular and has been used in numerous papers. In the context of CFD-DEM coupling, a common combination is that between ANSYS FLUENT and EDEM [92], [109], [111]-[113]. However, it should be noted that commercially available coupling modules are not currently designed for parallel computing [111]. This is a popular feature found in open-source and in-house developed software, which has proven to be quite promising [110], [114].

In contrast to commercial software, open-source software is not very user friendly and requires experience with various programming languages - e.g. C, $\mathrm{C}_{++}$, and FORTRAN - and computer operating systems - e.g. Linux. Regardless of this fact, opensource software is growing in popularity and has been used in several papers. In the context of CFD-DEM coupling, a common combination is that between OpenFOAM and LIGGGHTs [110], [115], [116].

Similar to open-source software, in-house developed software also requires a great deal of computer experience and programming abilities. However, it comes with the added advantage of greater flexibility and customization. This has proven to be a very desirable trait since in-house developed software has been used in several papers relating to CFD-DEM coupling [93], [117]-[119]. In addition to this, it has also been 
demonstrated that in-house developed software can be paired with commercial software [106]-[108], [120] - to lessen the programming and developmental burden.

\subsubsection{Advantages and Disadvantages}

CFD-DEM coupling currently provides the best trade-off between accuracy and computational efficiency in terms of capturing particle physics [14]. However, it should be reminded that this method has some shortcomings. The accuracy and applicability of CFD-DEM coupling is limited by the current state of the fluid-particle interaction force models. These equations are still in the developmental phase and require more thorough investigation and refinement [13]. In addition to this, the method is still somewhat computational intensive since it accounts for particle-particle and particle-wall interactions in a realistic manner. This limitation has restricted the CFD-DEM method to intermediatesized systems [16]. Additionally, it should be reminded that the applicability of the CFDDEM method is limited by the number of particles and the mesh element size. Such that, the latter is selected based on the particle size and geometry width [100]. When simulating, systems with large particles, the mesh size may even be too coarse to accurately predict the fluid flow field. However, these restrictions should not be viewed as unredeemable qualities. This method still has the potential to provide valuable insight within the restricted scope. 


\subsection{CFD-DEM Applications}

The application of CFD-DEM coupling to simulate solid-liquid systems is fairly wide spread. In this regard, fluidization, hydraulic conveying, separation (i.e. through centrifugation, sedimentation, filtration, and jigging) and mixing have been studied using this simulation method. A brief literature review of these applications is presented below.

\subsubsection{Fluidized Beds}

Fluidized beds are the most common system being studied with CFD-DEM coupling. Fluidization is the process of suspending particulate matter in an upward flow of a fluid phase. The purpose being to maximize the particle contact area and mixing performance. The studies using CFD-DEM to simulate solid-liquid fluidized beds cover a variety of subjects. This includes the hydrodynamic behavior of fluidized beds, the significance of various forces in fluidized beds, the layer inversion phenomenon occurring in polydisperse systems, and the influence of geometry on fluidization. In addition to this, there are also studies that focused on very specific topics such as heat transfer and magnetic fluidized beds.

The hydrodynamic behavior of fluidized beds has only been the subject of a few CFD-DEM studies. In this regard, Di Renzo and Di Maio [119] studied a pseudo-2D fluidized bed operating in the homogeneous expansion regime. With the aid of the simulations, the authors investigated the relationship between the superficial liquid velocity and the steady-state bed porosity. In doing so, they found that the steady-state 
bed porosity increased with increasing superficial liquid velocity. The simulation results qualitatively reproduced steady-state and transient behaviors in accordance with the theory of stability proposed by Foscolo and Gibilaro [121]. In addition to this, the simulation results also quantitatively reproduced the bed rise velocity as calculated by the particle bed model (PBM) [122]. This work demonstrated that CFD-DEM coupling could be used to reproduce the hydrodynamic behavior of a fluidized bed operating in the homogeneous expansion regime. Similarly, Wang et al. [93] studied the hydrodynamic behavior of a 2D fluidized bed. Although, they investigated the system in more detail by evaluating the influence of various operating conditions on the bed height and porosity, as well as the granular temperature - in not only the homogeneous expansion regime, but also the heterogeneous flow regime. In doing so, they found that the monitored parameters were influenced by the superficial liquid velocity, liquid viscosity, and particle density. Han et al. [116] studied the hydrodynamic behavior in a circulating fluidized bed. Their work comprehensively evaluated this system with a focus on the particle residence time and onset velocity. In essence, this is the velocity at which fluidization transitions from the conventional fluidization regime to the circulating fluidization regime. The developed CFD-DEM model was able to quantitatively reproduce experimental onset velocities found in literature (i.e. with an error ranging only from $0.6 \%$ to $2.1 \%$ ). Moreover, they evaluated the sensitivity of the onset velocity to changes in the riser dimensions and particle properties (i.e. size, density, contact parameters). In doing so, they found that only the particle size and density significantly influenced the onset velocity.

CFD-DEM coupling has also been used to evaluate the significance of various forces in solid-liquid fluidized beds. In this regard, Wang et al. [93] investigated the 
influence of including the lubrication force on their simulation results of a 2D fluidized bed. This force can hinder particles from moving towards or away from each other. The authors took this force into consideration by using a fluid-particle interaction force model. In doing so, they found that the CFD-DEM models that included the lubrication force produced more uniform porosity distributions and lower granular temperatures than the simulations that excluded the force. Moreover, the simulation results were found to be in good agreement with experimental results in terms of the particle axial velocities. However, the porosity was found to be overestimated towards the bed walls. The authors attributed this deviation to the 2D simplification of their model and the unrealistically constant coefficient of restitution used in their simulations. In regards to the latter, the authors suggested that the coefficient of restitution should realistically vary based on the influence of the lubrication force. This was taken into consideration in the work of Lui et al. [123], which comprehensively investigated the effect of the lubrication force on a $2 \mathrm{D}$ fluidized bed operating in the homogeneous expansion and heterogeneous flow regimes. In their study, the lubrication force was accounted for with a dynamic coefficient of restitution that was a function of the particles' Stokes numbers. When taking this force into consideration, the authors observed a decrease in the bed height and pressure drop. In addition to this, they observed that the lubrication force became more significant with decreasing relative velocities and particle sizes. Moreover, they were able to successfully reproduce the particle velocity distributions. Alternatively, Peng et al. [124] studied the forces acting on a foreign particle that was introduced into a fluidized bed containing particles with a lower density. Employing their validated CFD-DEM model, they evaluated this system based on the foreign particle's collision frequency and classification velocity. Such that, the latter 
is the constant velocity at which the foreign particle settles through the fluidized bed. Their results were found to be in agreement with theoretical expectations and experimental results measured using particle image velocimetry (PIV). Although, some discrepancies were observed, however, they attributed these to the ideal conditions used in their model and experimental measurement errors. Using their model, they found that the dominant force influencing the particle classification velocity changed with the bed porosity. Such that, the particle collision force was dominant in the regions with low bed porosities and the fluid drag force was dominant in the regions with high bed porosities. It was also found that the particle collision frequency increases with decreasing bed porosity and increasing particle size ratios (i.e. between the foreign particle and the fluidized bed particles). Using these results, the authors then proposed a correlation to predict the particle collision force based on the bed porosity.

The influence of particle polydispersity on the behavior of solid-liquid fluidized beds has also been studied using CFD-DEM coupling. In this regard, Malone et al. [125] attempted to reproduce the layer inversion phenomenon experienced in pseudo-2D fluidized beds containing polydisperse particles. Essentially, this phenomenon is experienced when fluidizing binary mixtures of particles that are composed of $(A)$ large and low-density particles and (B) small and high-density particles. At low superficial liquid velocities, the type A particles will form a layer on top of the type B particles. By increasing the superficial liquid velocity, the layers will invert at a critical inversion velocity. Some degree of segregation was observed in their work. However, their model was not able to reproduce the layer inversion phenomenon. The authors attributed this to their system geometry in which a pseudo-2D fluidized bed was used to combat computational costs. 
However, it should be noted that the CFD-DEM models applied by Cello et al. [117] and Di Renzo et al. [118] also used pseudo-2D geometries and they were able to successfully reproduce the layer inversion phenomenon. These authors suggested that the deviation in the work of Malone et al. [125] was more likely due to the use of a monodisperse drag model for polydisperse particles. Cello et al. [117] used a polydisperse drag model proposed by Di Maio et al. [126]. In doing so, they were able to quantitatively reproduce the experimental critical bed porosity at the inversion point. However, the critical velocity obtained from the model was found to be overestimated compared to the experimental value. They attributed this error to potential differences in the experimental and simulation conditions. More specifically, differences in the temperature and particle size distribution. In addition to this, another source of error that they noted was the inability of their simulations to reach a fully developed steady-state condition (i.e. simulations were very computationally intensive). Alternatively, Di Renzo et al. [118] used a polydisperse drag model proposed by Cello et al. [127]. Their results were found to be in good agreement with various experimental case studies found in literature. In this regard, their model was able to accurately reproduce both the critical velocities and bed porosities at the inversion points. The authors noted that differences in the experimental and simulation temperatures would have significantly influenced the accuracy of the predicted critical values. They found that minor changes in temperature resulted in significant changes in the critical bed voidage and minor changes in the critical velocity. In addition to this, they also confirmed that using a monodisperse drag model would have resulted in inaccurate behavior. 
CFD-DEM coupling has also been used as a tool to predict the influence of bed geometry on the fluidization quality. In this regard, Al-Arkawazi et al. [128] compared the fluidization quality in various $2 \mathrm{D}$ bed geometries. These included a simple column, a column with a vertical plate, and a column with two symmetric lateral inlets. They facilitated this comparison with a novel fluidization indicator, which was proposed to better characterize the fluidization state. In this comparison, they found that the fluidization quality was reduced by the addition of a vertical plate and not significantly influenced by the addition of the lateral inlets. It should be noted that these results were not validated against experimental data. Therefore, their analysis was limited to a qualitative comparison.

The versatility of the CFD-DEM method facilitates the modification of the governing equations to account for heat transfer and non-contact forces such as magnetic or electrostatic forces. However, this does not fall under the current scope. The reader is referred to the works of Malone and Xu [129] and Orona et al. [130], which investigated heat transfer in solid-liquid fluidized beds via CFD-DEM coupling. In addition to this, refer to the works of Wang et al. [131], [132] for CFD-DEM applications involving the influence of magnetic fields on solid-liquid fluidized beds.

\subsubsection{Hydraulic Conveyance}

The CFD-DEM approach has been used to simulate hydraulic conveying systems. Hydraulic conveying is the process of transporting particulate matter within a carrier fluid. 
The CFD-DEM studies investigating this process have covered a variety of system geometries such as channels, pipes, and elbows.

The hydrodynamic behavior occurring within hydraulic conveying systems has been the focus of the majority of the CFD-DEM studies investigating such systems. In this regard, Lim [133] studied the formation and propagation of voidage waves in pseudo-2D vertical pipes and horizontal channels. Pseudo-2D geometries were adequate in reproducing the voidage wave instability phenomenon. However, as the author noted, these geometries could not produce representative packing structures. Therefore, they limited their study to a qualitative analysis. In this regard, the author evaluated the sensitivity of the voidage wave frequency and shape to changes in the particle concentration and liquid velocity. In doing so, it was found that the voidage wave instability was independent of the liquid velocity in vertical pipes, but not in horizontal channels. Bravo-Blanco et al. [134] studied the incipient motion of single particles in an open channel. They evaluated this system based on the critical liquid velocity in which particle motion was initiated. Their CFD-DEM simulation results were found to be in good agreement with experimental results measured using acoustic Doppler velocimetry (i.e. with an error of less than $5 \%$ ). The authors attributed the minor deviation to the ideal conditions used in their simulations. Through their experimental and numerical efforts, it was found that the critical liquid velocity increased linearly with increasing particle size. Redlinger-Pohn et al. [135] studied the suspension of elongated particles in a closed torus. This was done in order to approximate the flow of fibers in a coiled tube. In their simulations, they used a novel technique to reduce their computational costs. This was facilitated by splitting the coupled forces and torques into explicit and implicit terms, which 
allowed larger coupling intervals to be used. The CFD-DEM model results were verified against analytical solutions. The authors note that their code captured the expected particle behavior with increasing particle density, but not with increasing particle Reynolds number. This limited their study to the creeping flow regime. In their simulations, it was found that the particle fibers segregated based on the fiber length.

The effects of the lubrication force on CFD-DEM simulation results were also investigated in some of the hydraulic conveying studies. In this regard, Lim [133] investigated the sensitivity of the voidage wave instability to changes in the particles' coefficient of restitution in a pseudo-2D vertical pipe. Refer to the experimental works of Joseph et al. [136] for the relationship between the lubrication force and the coefficient of restitution. In the sensitivity test performed by Lim [133], it was found that the tendency for the voidage wave instability to be formed decreased with increasing inelasticity (i.e. lower coefficients of restitutions). The author reported this finding to be anomalous. However, they did not take the relationship between the coefficient of restitution and the lubrication force into consideration. When taking this relationship into account, this finding is actually reasonable. Based on the experimental work of Joseph et al. [136], lower coefficients of restitution should correspond to greater lubrication forces. This should then promote greater uniformity as supported by the work of Wang et al. [93]. Taking into consideration that voidage waves are visually recognized as accumulations of dilute and dense sections of particles, greater uniformity will be recognized as diminished voidage waves. In summary, it was realistic for Lim [133] to observe a decrease in the tendency for voidage waves to be formed based on additional literature. Tomac and Gutierrez [137] studied the effect of the lubrication force on particle motion in a 2D horizontal channel. 
The authors took this force into consideration by modifying the particle contact model. In doing so, it was observed that agglomeration would occur with greater lubrication forces. This behavior is in stark contrast to the lubrication force studies discussed in Section 2.6.1. However, this deviation is most likely due to a limitation associated with their lubrication force model. Specifically, the fact that the lubrication force was allowed to approach infinity as the surface distance approaches zero (i.e. "Stokes Paradox"). This prevents particles from truly contacting and therefore rebounding. Refer to the work of Zhang and Horio [97] for an overview of this issue and the methods used to avoid it. In this regard, it should also be noted that the previously discussed studies - that focused on the effect of the lubrication force - did account for the "Stokes Paradox". Wang et al. [93] avoided this issue by implementing a minimum approachable surface distance. Whereas, Lui et al. [123] intrinsically avoided the issue by taking the lubrication force into consideration through the particles' coefficient of restitution and Stokes number. Thereby, avoiding the use of the surface distance parameter.

The wear and damage resulting from particle-geometry contacts in hydraulic conveying systems has been the subject of a few CFD-DEM studies. In this regard, Tan et al. [138] investigated the wear patterns in a $90^{\circ}$ elbow, which was used to transport a slurry (i.e. composed of a binary mixture of particles varying only in size and a viscous liquid). In their simulations, they predicted the location in which the maximum erosive wear damage would be experienced (i.e. the puncture point location) by evaluating the particle-wall collision frequency and intensity. Their results were in qualitative agreement with industrial observations. Zhang et al. [139] extended the CFD-DEM model developed by Tan et al. [138] and investigated the influence of various parameters on the puncture 
point location in a $90^{\circ}$ elbow. However, their prediction of the puncture point location was only based on the particle-wall impact force. When increasing the slurry velocity, it was found that the puncture point location shifted downstream and the impact force experienced at the puncture point increased. This was in qualitative agreement with experimental observations found in literature. Chen et al. [113] also investigated the wear patterns in elbows. However, they focused on the influence of the elbow angle (i.e. $45^{\circ}$, $60^{\circ}, 90^{\circ}$ ) on the erosion rate. In their CFD-DEM simulations, they estimated the erosion rate with the particle-wall impact velocity and impingement angle. The location of the maximum erosion rate for the $90^{\circ}$ elbow was found to be in agreement with experimental observations found in literature. Using this model, it was also found that the maximum erosion rate increases with increasing elbow angle, but is consistently located near the exit of the elbow. Furthermore, the simulation results showed that the particle motion was dominated by drag and gravitational forces in the $45^{\circ}$ and $60^{\circ}$ elbows. While, in $90^{\circ}$ elbows, turbulence and secondary flows dominated the particle motion. Alternatively, Azimian et al. [140] investigated the wear on a cylindrical rod obstructing flow in a horizontal pipe. Their one-way coupled CFD-DEM model was able to reproduce the velocity profiles (i.e. before and after the obstruction) in good agreement with experimental results measured using Laser Doppler Anemometry (LDA). Some discrepancies were observed in the velocity profile measured in front of the obstruction. The authors attributed this to a need for further grid refinement. Using the same method as Chen et al. [113], they were able to predict the erosion rate acting on the cylindrical obstruction. In doing so, it was found that the erosion rate increased with increasing flow rate and solids concentration. 


\subsubsection{Separation and Classification}

Separation processes have also been studied using CFD-DEM coupling. These processes are responsible for removing particulate matter from solid-liquid mixtures. The studies using CFD-DEM to investigate these processes have covered a variety of unit operations. These include sedimentation tanks, filters, hydrocyclones, centrifuges, and jigs.

The cake formation and growth in sedimentation tanks and filters have been the subject of a few of these studies. In this regard, Dong et al. [141] evaluated the influence of various operating conditions on the cake porosity in a pseudo-2D sedimentation tank. Their one-way coupled CFD-DEM model was able to qualitatively reproduce the cake growth behavior in agreeance with theoretical expectations found in literature. Using this model, it was then found that the cake porosity in sedimentation tanks increased with increasing Van der Waals forces, liquid viscosity, and liquid density; as well as decreasing particle size. In a later work, Dong et al. [142] quantitatively validated their model based on experimental results. Such that, the predicted cake porosities (i.e. for varying particle sizes as well as liquid viscosities and densities) were found to be comparable to the measured values. Dong et al. [143] then applied their model to simulate a pseudo-2D vertical filtration system. In doing so, it was observed that the cake porosity - on the filter surface - also increased with increasing Van der Waals forces and decreasing particle size. However, it decreased with increasing liquid viscosity and density. This behavior is in contradiction to that observed in sedimentation tanks. The authors attributed this difference to the increase in fluid-particle interaction forces, which resulted from 
increasing the liquid viscosity and density. The fluid-particle interaction forces acted in different directions in the sedimentation tank and filtration system. Such that, these forces acted in the same direction as gravity in the filtration system and in the opposite direction in the sedimentation tank. This resulted in a decrease in cake porosity in the filtration system and an increase in the sedimentation tank. Sören and Jürgen [144] also studied the hydrodynamic behavior of a pseudo-2D filtration system. However, they investigated the effects that flocculation has on the cake properties. By comparing the simulation results of stable suspensions to that of flocculated suspensions, it was found that the flocculated particles produced greater cake porosities and permeabilities. Their predicted cake porosities were in good agreement with their experimental values (i.e. with an error ranging from $4-9 \%)$. The authors attributed the minor deviation to the pseudo-2D simplification made in their model. Zhao et al. [105] studied the hydrodynamic behavior of a batch sedimentation tank. They successfully verified their CFD-DEM model against an analytical solution of a single particle settling. Using this model, they were able to qualitatively reproduce the particle segregation and consolidation behavior presented in literature. Moreover, their predicted maximum pore water pressure (i.e. resulting from particle consolidation) was found to be in good agreement with the analytical solution.

CFD-DEM coupling has also been used to model hydrocyclones and centrifuges. In this regard, Chu et al. [145], [146] investigated the influence of particle concentrations and density distributions on the separation efficiency of a hydrocyclone. The authors limited their studies to qualitative analyses due to the simplifications made in their models. More specifically, those related to particle shape and size, and the use of the parcelparticle approximation in [146]. With the one-way coupled CFD-DEM model used in [145], 
it was found that the separation efficiency of the hydrocyclone deteriorated with increasing particle concentrations - due to the associated increase in particle-particle collisions. With the two-way coupled CFD-DEM model used in [146], it was also found that the separation efficiency of fine particles deteriorated as the averaged particle density was increased. The authors suggested that this behavior was due to the related reduction in the tangential velocity of the fluid phase. Alternatively, Fernández et al. [112], [147] studied the build-up and behavior of particle sediments in a centrifuge system. Their CFDDEM model was able to qualitatively reproduce the theoretically expected particle trajectories and sediment build-up patterns. Using this model, the authors found that the sediment build-up pattern was influenced not only by the rotational velocity of the centrifuge bowl, but also by the particle sliding friction coefficient [112]. This suggests that particle-particle and particle-wall interactions played a significant role in the sediment build-up pattern. Moreover, it was demonstrated that the sediment build-up influenced the fluid flow patterns [112], [147]. Zhou et al. [88] investigated the influence of the various forms of CFD-DEM coupling equations on the accuracy of modeling a hydrocyclone. Refer to Section 2.5.1 for a review of these equations. From their simulations, it was found that the simplified version of Model B could not reproduce the expected particle behavior (i.e. separation based on the particle density). The authors attributed this to the unsteady and non-uniform flow occurring within the hydrocyclone, which violated the assumptions under which the model was derived. Alternatively, it was demonstrated that Model B, in its original form, was capable of reproducing the expected particle behavior. For this reason, the authors recommended that the simplified version of Model B should not be used in future works. 
Several of the CFD-DEM studies investigating separation processes focused on jigging operations. Jigging is the process of separating and classifying particulate matter by means of agitation within a pulsated liquid flow. Such that, the pattern in which the liquid is pulsated is known as the jigging profile. In this regard, Xia et al. [104] investigated the hydrodynamic behavior of a 2D batch jig with a sinusoidal jigging profile. Using their CFD-DEM model, particle stratification was observed as a result of the pulsated flow. This was in qualitative agreement with the expected behavior of a jig. Xia and Peng [148] continued this work and investigated the influence of the jigging amplitude and frequency on the separation efficiency. From their simulations, it was found that the separation efficiency increased by increasing both the amplitude and frequency. Such that, the latter had a more pronounced effect. Although, it should be noted that increasing either of these parameters, only increased the separation efficiency up to an optimal value - after which unstable separation occurred. Moreover, it was found that the separation efficiency was also affected by the particle size and density. Dong et al. [120] also evaluated the sensitivity of the separation efficiency to changes in the jigging profile. Although, this was investigated in a 3D in-line pressure jig (IPJ) with a jig-saw jigging profile. Their CFD-DEM model was able to qualitatively reproduce the experimental trends of the separation efficiency. The authors attributed the quantitative deviation to a need for model calibration. In their simulations, it was confirmed that the separation efficiency increased with increasing jigging amplitudes and frequencies. Alternatively, Viduka et al. [106]-[108] studied the sensitivity of the separation time to changes in the jigging profile. This was done in a pseudo-2D batch jig. By using sinusoidal and trapezoidal jigging profiles, it was found that increasing the amplitude and frequency of the jigging profile decreased the 
separation time. Such that, it decreased exponentially with changes in the sinusoidal profile [107] and linearly with changes in the trapezoidal profile [108]. Additionally, it should be noted that increasing the amplitude or frequency, only decreased the separation time up to an optimal value - after which unstable separation would take place. By comparing these jigging profiles to others, it was also found that the shape of the jigging profile influenced the particle flow pattern [106]. Based on these results, the authors proposed several ways in which jigging operations could be optimized (i.e. to minimize the separation time, mechanical wear, and energy consumption).

\subsubsection{Solid-Liquid Mixing}

To a lesser extent, CFD-DEM coupling has also been applied to solid-liquid mixing systems. The majority of these studies have focused on the hydrodynamic behaviors occurring within solid-liquid stirred tanks. In this regard, Misumi et al. [149] investigated the influence of different impeller clearances, and the presence of baffles, on the particle motion in a tank filled with $0.1 \mathrm{~mm}$ glass beads and water. Using their CFD-DEM model, it was observed that varying the mixing system configuration changed the stagnant regions in which particles would accumulate on the bottom of the tank - before becoming suspended. Alternatively, Srinivasa and Jayanti [150] investigated the influence of various process parameters on the just-suspension speed. Their simulation results were in qualitative agreement with experiment trends for varying particle size, density, sphericity, and impeller type. These trends were based on mixtures composed of various irregularly shaped particles and water (i.e. sand, $\mathrm{CaSO}_{4}$, and $\mathrm{CaO}$ mixtures). However, their model 
was not able to accurately reproduce the degree of mixing homogeneity, a key characteristic of mixing. The authors attributed this to the applied turbulent and drag equations. As discussed by the authors, the standard k- $\varepsilon$ turbulence model only considers large turbulent eddies and not smaller eddies responsible for further particle dispersion and entrainment. Boucher et al. [151] used similar turbulence and drag equations, when studying the motion of quartz particles in a tank of water. However, their CFD-DEM model was able to quantitatively reproduce the range of particle velocities and displacements. Specifically, when compared to experimental data obtained using Positron Emission Particle Tracking (PEPT). This was possible due to their significantly lower particle concentration. Shao et al. [92] investigated the influence of the Magnus lift force on the hydrodynamic behaviors of $0.3 \mathrm{~mm}$ glass beads suspended in water. In their CFD-DEM simulations, it was found that the Magnus lift force was significant compared to the drag force. The authors attributed this to the high rate of particle-particle and particle-wall collisions. Derksen [98] also verified this using a LES-DEM model. Furthermore, Shao et al. [92] was able to quantitatively reproduce the spatial distribution of the particle velocities in good agreement with experimental results - measured using Computer Automated Radioactive Particle Tracking (CARPT). In addition to this, they also evaluated the degree of particle dispersion through the examination of the spatial distributions of the particle concentrations. However, it should be noted that they did not use mixing indexes and also did not validate these results directly. The latter limited their interpretation to a qualitative analysis. On a different note, Blais et al. [102], [103], [152] investigated the fraction of suspended particles in a tank filled with $3 \mathrm{~mm}$ glass beads and a viscous glucose solution. In their studies, the flow regime was varied from the laminar regime to the early turbulent 
regime. Their simulation results were found to be in good agreement with experimental values obtained using the pressure gauge technique. In the laminar to transitional regimes, they evaluated the sensitivity of their CFD-DEM model over a range of particle properties. In doing so, they found that the sliding and rolling friction coefficients played a key role in the degree of particle suspension. Alternatively, modifying the Young's Modulus and coefficient of restitution had almost no effect [103]. In the early turbulent regime, they investigated and accurately reproduced the minimum speed for justsuspension [152]. However, throughout their studies, they only took particle suspension into consideration and did not account for the particle distribution.

It should be noted that CFD-DEM coupling is not commonly used to model solidliquid mixing in stirred tanks. In literature, these systems have conventionally been modeled with TFM [5]-[12]. However, as previously stated, this method is less accurate at accounting for particle-particle and particle-wall interactions - which have been found to significantly influence the hydrodynamic behavior of solid-liquid mixing. In this regard, Shao et al. [92] demonstrated that particle-particle and particle-wall interactions influenced the distribution of particle concentrations. By accounting for the Magnus force, it was observed that the particles would lift due to the high rates of particle-particle and particle-impeller interactions, which produced large particle angular velocities - and as a result large Magnus forces. In addition to this, Blais et al. [103] demonstrated that particleparticle interactions influenced the degree and rate of particle suspension. By increasing the sliding and rolling friction coefficients, it was observed that the fraction of suspended particles increased (i.e. at intermediate impeller speeds), and steady-state was reached faster (i.e. at all impeller speeds). These findings suggest that CFD-DEM coupling is the 
more appropriate modeling method - when compared to TFM - since it can more accurately account for the particle physics responsible for certain hydrodynamic behaviors observed in solid-liquid mixing systems. For this reason, this numerical method was selected for the current work. Although, it should be reminded that this will come at a higher - but manageable - computational cost and greater system limitations.

\subsection{Research Objectives}

From the literature review, provided above, it is apparent that research gaps exist in both the application of CFD-DEM coupling and ERT to solid-liquid mixing systems. This study will attempt to close these knowledge gaps. The main objectives of this work are as follows:

i. To understand the limitations of applying the CFD-DEM coupling method to turbulent solid-liquid mixing systems.

ii. To develop a CFD-DEM model using FLUENT and EDEM to enable the prediction of mixing quality.

iii. To perform a more rigorous assessment of the developed CFD-DEM model, based on the spatial distribution of the particle concentrations, by comparing the simulation results to experimental data obtained using ERT.

iv. To apply mixing index definitions, previously only developed in literature to analyze ERT data, to the CFD-DEM simulation results in order to provide a more convenient and meaningful method of assessment. 
v. To develop a new mixing index definition to quantify the degree of suspension to work in tandem with the existing dispersion mixing indexes and provide a more complete approach of interpreting mixing quality.

vi. To evaluate the sensitivity of the mixing quality, as predicted by the developed CFD-DEM model, to changes in the various model parameters in order to find areas of improvement within the model. 


\section{Chapter 3 : Experimental Methodologies}

The experimental set-up used in the current work is described in this chapter with a detailed discussion on the ERT system and its components. Details of the methods used to analyze the ERT data and the experimental results are also discussed within this chapter.

\subsection{Experimental Set-up}

The mixing system used in this study consisted of a flat-bottom cylindrical tank, four equally spaced baffles, and a downward-pumping pitched blade turbine (PBT) impeller. The impeller is rotated in the clockwise direction. Refer to Figure 4 for a schematic diagram of this set-up. The stirred tank diameter $(T)$ and liquid level height $(H)$

were both $25.1 \mathrm{~cm}$. While, the impeller diameter (D) and clearance $(C)$ were $10.3 \mathrm{~cm}$ (T/2.4) and $6.3 \mathrm{~cm}(T / 4.0)$, respectively. The baffle width, thickness, and clearance were 
$2.1 \mathrm{~cm}(\mathrm{~T} / 12), 0.3 \mathrm{~cm}(\mathrm{~T} / 84)$, and $0.6 \mathrm{~cm}(\mathrm{~T} / 42)$, respectively. These dimensions were appropriate based on the tank diameter to dimension ratios, which were relatively close to typical values used in literature [19], [20]. The impeller was driven by a LIGHTNIN series LabMaster, which also acted as the measurement device for the impeller speed and torque. During experiments, the impeller speed was varied in 50 RPM increments ranging from 250 RPM to 550 RPM. Each trial was repeated in triplicate in order to ensure the repeatability of the experiments. This was proven to be acceptable based on the error bars depicted in the experimental results presented in Section 3.4.

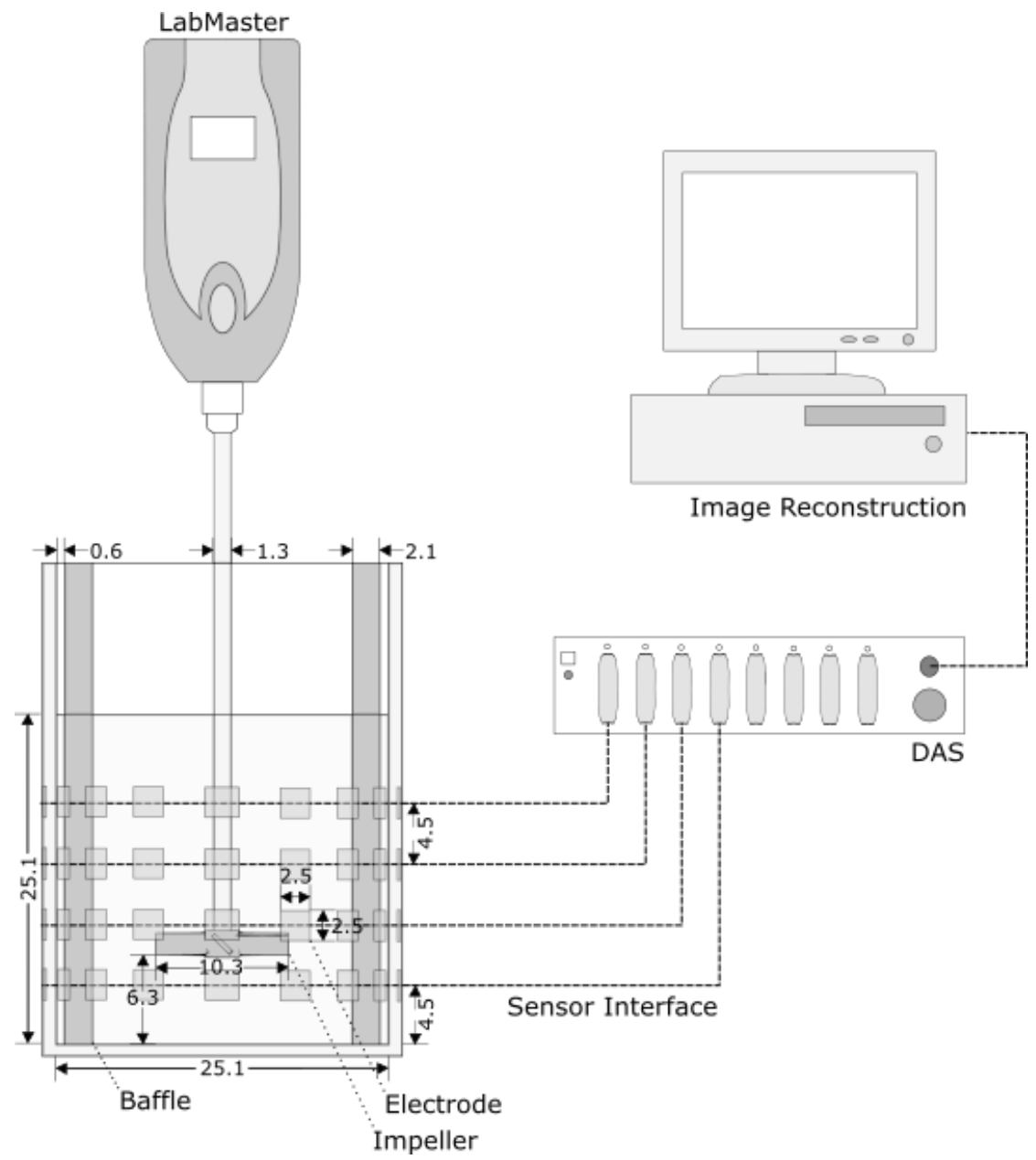

Figure 4. Schematic diagram of experimental set-up. 
This system was used to suspend a mixture of tap water and glass impact beads. Refer to Table 4 for the experimental operating conditions. In order to determine the spatial distribution of this mixture, the set-up was also equipped with a commercial ERT system manufactured by Industrial Tomographic Systems Limited. This system will be discussed in Section 3.2 and has also been depicted in Figure 4.

Table 4. Experimental operating conditions.

\begin{tabular}{|c|c|c|c|}
\hline \multicolumn{1}{|c}{ Parameter } & Variable & Value & Unit \\
\hline Liquid Phase & $\mathrm{N} / \mathrm{A}$ & Tap Water & $\mathrm{N} / \mathrm{A}$ \\
\hline Solid Phase & $\mathrm{N} / \mathrm{A}$ & Glass Beads & $\mathrm{N} / \mathrm{A}$ \\
\hline Particle Diameter & $\mathrm{d}_{\mathrm{p}}$ & 2 & $\mathrm{~mm}$ \\
\hline Particle Weight Fraction & $\mathrm{w}_{\mathrm{p}}$ & 5 & $\mathrm{wt} \%$ \\
\hline Impeller Speed & $\mathrm{N}$ & $250-550$ & $\mathrm{RPM}$ \\
\hline
\end{tabular}

\subsection{ERT Measurement System}

A basic ERT measurement system is comprised of three components: the sensor interface, the data acquisition system (DAS), and the data processing software. In the current work, the sensor interface was used to inject current into the mixing tank and detect the change in voltage. It was comprised of 1 " $\times 1$ " stainless steel electrodes arranged into four sensing planes. Each plane contained 16 electrodes that were equally spaced apart in a ring. The planes were then spaced $4.5 \mathrm{~cm}$ apart with the lowest plane $4.5 \mathrm{~cm}$ from the bottom of the tank. Such that, the planes were numbers from the top to the bottom. 
The electrodes were then connected to the DAS with a series of co-axial cables in order to minimize environmental noise and interference [39]. This component managed the measurement process. Specifically, this was done based on the adjacent data collection scheme and the settings listed in Table 5. In this data collection strategy, neighboring electrodes were grouped into pairs and each measurement was taken with adjacent electrode pairs such that one pair was used to inject current into the system and the other was used to detect the resulting output voltage [153]. The electrode pairs were alternated until all independent measurements were taken.

Table 5. Settings used for ERT measurement.

\begin{tabular}{|c|c|c|}
\hline Parameter & Value & Unit \\
\hline Injection Current Amplitude & 13 & $\mathrm{~mA}$ \\
\hline Injection Current Frequency & 9600 & $\mathrm{~Hz}$ \\
\hline Sampling Time Interval & 60 & $\mathrm{~ms}$ \\
\hline Samples per Frame & 8 & $\mathrm{~N} / \mathrm{A}$ \\
\hline Number of Reference Frames & 50 & $\mathrm{~N} / \mathrm{A}$ \\
\hline Maximum Number of Frames & 30 & $\mathrm{~N} / \mathrm{A}$ \\
\hline Frames per Download & 1 & $\mathrm{~N} / \mathrm{A}$ \\
\hline
\end{tabular}

After the measurement data was collected, the image reconstruction software was used to generate tomographic images. These images illustrated the spatial distribution of the particle concentrations. Refer to Figure 5 for sample images - these depict the timeaveraged particle concentrations obtained over a span of 30 frames, after steady-state was reached. 
a)

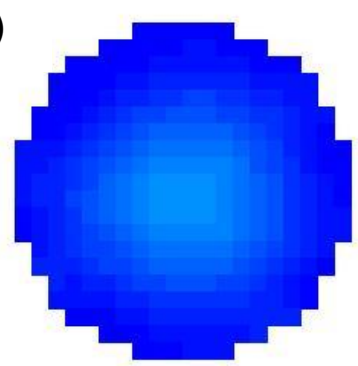

Plane 1

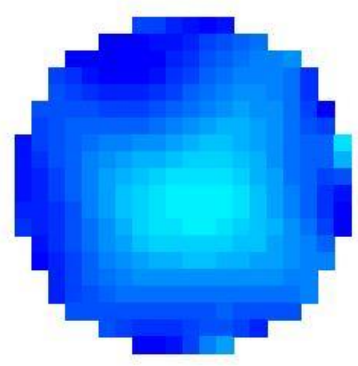

Plane 3

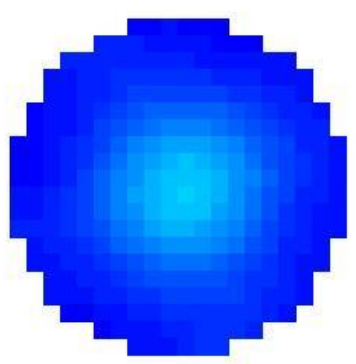

Plane 2

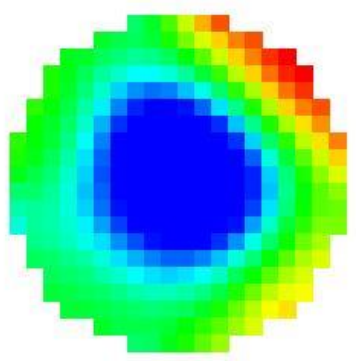

Plane 4

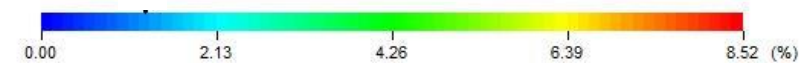

b)

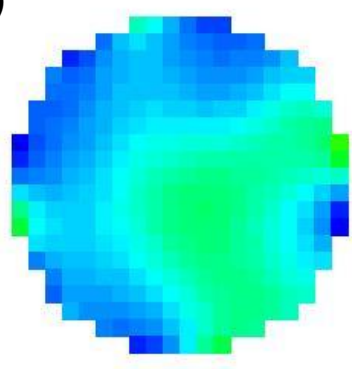

Plane 1

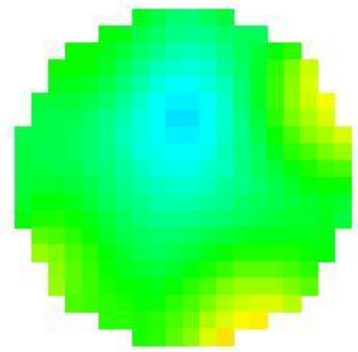

Plane 3

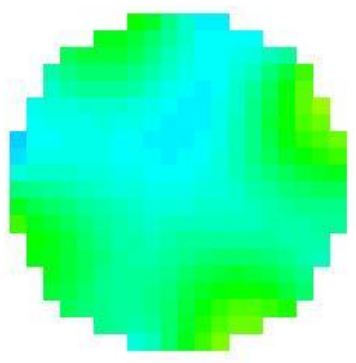

Plane 2

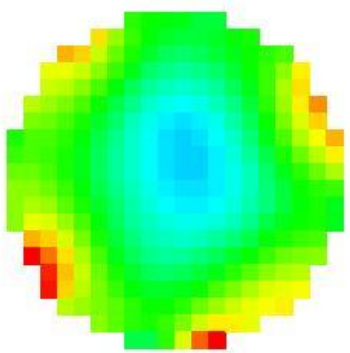

Plane 4

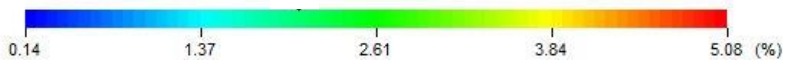

Figure 5. ERT particle concentration tomographs - time-averaged for 30 frames - at impeller speeds of a) 250 RPM and b) 550 RPM. 
In the current work, the tomographic images were generated by first using the finite difference method and the non-iterative linear back-projection method to solve for the conductivity distribution. The linear back-projection method consists of a two-step algorithm: solving the forward problem and then solving the inverse problem. The forward problem consists of determining a sensitivity map of the discretized measurement plane. This map can be defined as follows [154]:

$$
S_{c u, v o, x, y}=\int \frac{\nabla^{2} V_{c u}}{I_{c u}} \frac{\nabla^{2} V_{v o}}{I_{v o}}
$$

where $c u$ corresponds to the current-driven electrodes, vo corresponds to the voltage measurement electrodes, $V$ is the voltage, and $I$ is the injection current. Such that, the voltage gradients are determined by solving equation ( 1 ) with a set of reference data. The inverse problem consists of actually determining the conductivity matrix. This is achieved by using the following equation:

$$
\sigma(x, y)=\frac{\sum_{c u}^{M} \sum_{v o}^{N} S_{c u, v o, x, y} \ln \left(\frac{V(x, y)}{V_{r e f}(x, y)}\right)}{\sum_{c u}^{M} \sum_{v o}^{N} S_{c u, v o, x, y}}
$$

where $\sigma$ is the conductivity, $M$ is the number of measurements, $N$ is the number of elements, and ref represents the reference readings. Even though this method may seem intricate, it is more direct and less computationally intensive than its iterative counterparts [42]. The conductivity distribution was then converted to a distribution of 
particle concentrations. This was achieved by using a simplified version of Maxwell's equation for non-conductive dispersed phases [39]:

$$
X=\frac{2 \sigma_{1 \mathrm{iq}}-2 \sigma_{m c}}{\sigma_{m c}+2 \sigma_{1 \mathrm{iq}}}
$$

where $X$ is the volume fraction, $\sigma_{1 i q}$ is the conductivity of the liquid phase, and $\sigma_{m c}$ is the measured conductivity.

\subsection{Quantification of Mixing Quality with Mixing Indexes}

When interpreting the tomographs, discussed in Section 3.2, the spatial distribution of the particle concentrations can only be interpreted visually. For this reason, they can only be compared qualitatively with other experimental and simulation results. A better point of comparison is referred to as a mixing index. This parameter summarizes the data in the tomographs into a single and easily comparable value. This value then acts as a measure of the uniformity of mixing. Its numerical definition can vary based on how the data is processed.

The different definitions are all related to the relative standard deviation of the local particle concentrations. The only difference is the regions, within the tomographs, that are used to define the local particle concentrations. This can be entire planes, concentric rings, or even individual elements. Refer to Table 6 for the related numerical definitions. Such that, $P$ represents plane $\mathrm{P}, R$ represents ring $\mathrm{R}, K$ represents element $\mathrm{K}, X_{P}$ is the average concentration in plane $\mathrm{P}, \overline{X_{P}}$ is the total average of all $X_{P}$ values, $X_{R, P}$ is the 
average concentration in ring $\mathrm{R}$ and plane $\mathrm{P}, \overline{X_{R, P}}$ is the total average of all $X_{R, P}$ values, $X_{K, P}$ is the concentration in element $\mathrm{K}$ and plane $\mathrm{P}, \overline{X_{K, P}}$ is the total average of all $X_{K, P}$ values, $X_{R, a v g}$ is the average of $X_{R, P}$ values in the same annular section $\mathrm{R}, \mathrm{X}_{K, \text { avg }}$ is the average of the $X_{K, P}$ values in the same element location, and $X_{P, a v g}$ is the average of the $X_{R, P}$ or $X_{K, P}$ values in the same plane $\mathrm{P}$.

\section{Table 6. Summary of mixing indexes used previously in literature.}

\begin{tabular}{|c|c|c|c|c|}
\hline $\begin{array}{l}\text { Local } \\
\text { Region }\end{array}$ & $\begin{array}{l}\text { Mixing } \\
\text { Quality }\end{array}$ & Mixing Indexes & & Authors \\
\hline Plane & Axial & $M I_{z}=1-\frac{1}{\overline{X_{P}}} \sqrt{\frac{\sum_{1}^{P}\left(X_{P}-\overline{X_{P}}\right)^{2}}{P-1}}$ & $(29)$ & $\begin{array}{l}\text { Hosseini et } \\
\text { al. [20] }\end{array}$ \\
\hline \multirow{3}{*}{ Ring } & Axial & $M I_{z}=\frac{1}{\overline{X_{R, P}} R} \sum_{1}^{R} \sqrt{\frac{\sum_{1}^{P}\left(X_{R, P}-X_{R, a v g}\right)^{2}}{P-1}}$ & $(30)$ & \multirow{3}{*}{$\begin{array}{l}\text { Harrison et } \\
\text { al. [4] }\end{array}$} \\
\hline & Radial & $M I_{r}=\frac{1}{\overline{X_{R, P}} P} \sum_{1}^{P} \sqrt{\frac{\sum_{1}^{R}\left(X_{R, P}-X_{P, a v g}\right)^{2}}{R-1}}$ & $(31)$ & \\
\hline & Overall & $M I_{t}=\frac{1}{\overline{X_{R, P}}} \sqrt{\frac{\sum_{1}^{R} \sum_{1}^{P}\left(X_{R, P}-\overline{X_{R, P}}\right)^{2}}{R P-1}}$ & $(32)$ & \\
\hline \multirow{2}{*}{ Element } & Radial & $M I_{r}=\frac{1}{\overline{X_{K, P}} P} \sum_{1}^{P} \sqrt{\frac{\sum_{1}^{K}\left(X_{K, P}-X_{P, a v g}\right)^{2}}{K-1}}$ & ( 33 ) & $\begin{array}{l}\text { Williams et } \\
\text { al. [45] }\end{array}$ \\
\hline & Overall & $M I_{t}=\frac{1}{\overline{X_{K, P}}} \sqrt{\frac{\sum_{1}^{P} \sum_{1}^{K}\left(X_{K, P}-\overline{X_{K, P}}\right)^{2}}{K P-1}}$ & $(34)$ & $\begin{array}{l}\text { Carletti et al. } \\
\qquad[47]\end{array}$ \\
\hline
\end{tabular}

When using individual elements to calculate a mixing index, the defining region is relatively small and the experimental measurements can be influenced by noise. This 
noise can be inherent to the measurement system, experimental set-up, or even the heterogeneous nature of the solid-liquid mixture. On the other hand, when averaging the tomograph elements across an entire plane to calculate a mixing index, the defining region is too large and the radial inhomogeneities resulting from poor mixing cannot be taken into consideration. In other words, the radial distribution of the particles cannot be studied by using plane-based mixing indexes. To avoid the aforementioned issues, the concentric ring definition will be used in this study. This definition allows the mixing quality to be evaluated in the radial and axial directions as well as the entire mixing tank (i.e. overall). In this study, only the overall mixing index will be used since it accounts for both the radial and axial distributions in one convenient value.

In this work, image reconstruction was performed using the finite difference method. As a result, the tomographs produced were forced to fit into a square grid of square elements. This was done regardless of the shape of the cross-sectional area being measured. Essentially, this was accomplished by allowing the square tomographic elements to either lie within or outside of the cross-sectional area. However, with this type of layout, the local particle concentrations cannot be defined based on concentric rings. Since, square elements cannot be combined to create perfect circles. For this reason, the zoning scheme, depicted in Figure 6, was used to approximate the concentric rings. Although, it should be noted that the zones - depicted in this figure - do not cover the same amount of surface area. However, this did not significantly influence the mixing index calculations. When using a weighted average to account for the varying sizes of the ring-areas, the resulting mixing indexes were found to be quantitatively similar to those obtained without using a weighted average. For this reason, the approach used in the 
current work can be considered to be valid and can also be used to simplify the mixing index calculations.

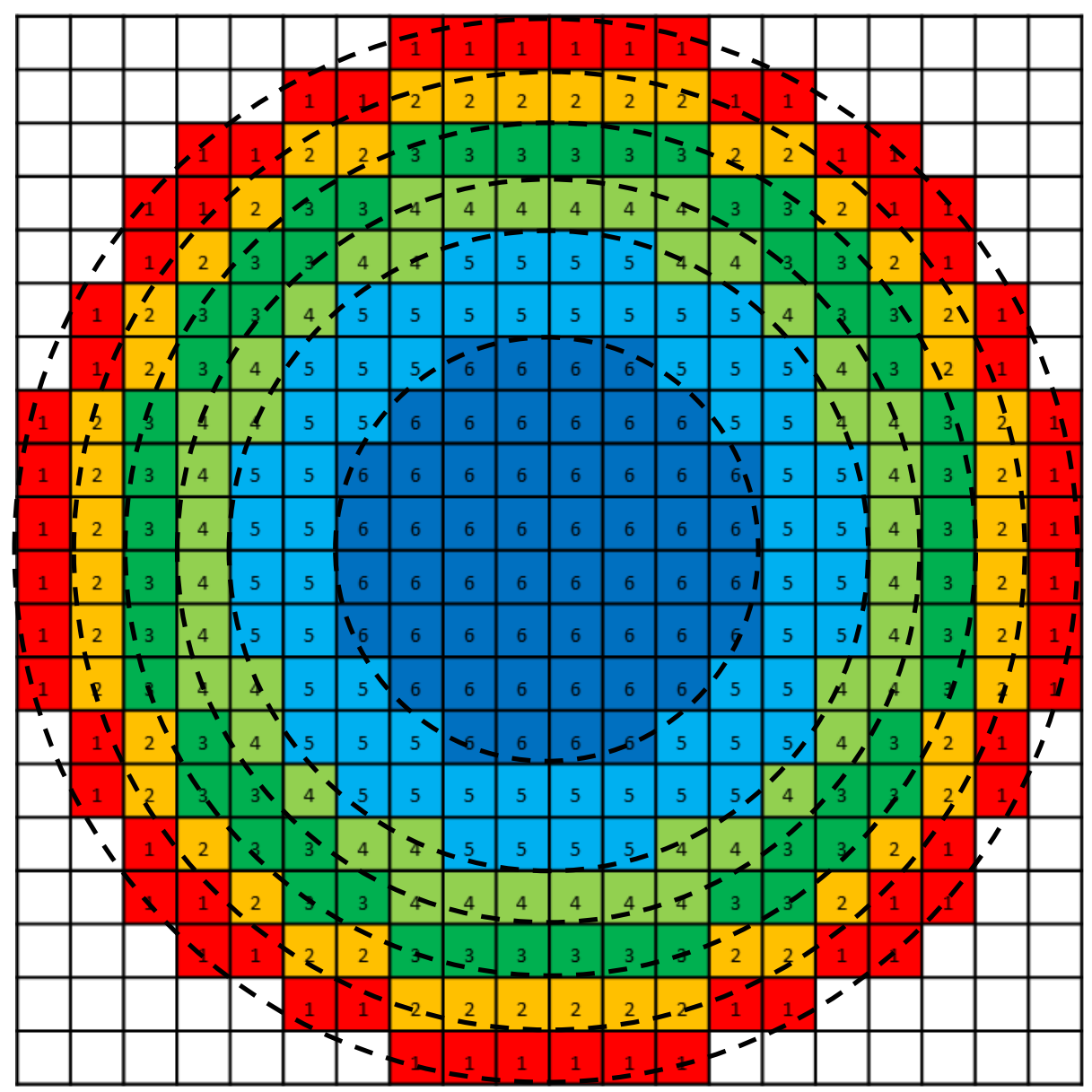

Figure 6. Quadrilateral ERT tomographic grid and zone scheme.

However, it is reminded that this mixing index only acts as a measure of how well the particles are dispersed within the tomograph planes. This does not always correlate to the mixing quality throughout the entirety of the system. Therefore, it is proposed that another mixing index be considered in order to account for the suspension of particles from the bottom of the tank. This approach is similar to the that taken by Blais et al. [102], [103], [152]. However, their mixing index was based on the pressure measured at the 
bottom of the stirred tank. In this work, the suspension mixing index will be defined based on the average of the measured local particle concentrations. In literature, local particle concentration values have been used previously as a measure of particle suspension. Specifically, in order to determine the minimum speed for just-suspension. This was done by Bourne and Sharma [155] using a direct sampling technique, Musil [156] using optical measurements, and Musil and Vlk [157] using invasive conductivity probes. However, in these studies, the local particle concentration was only monitored at one measurement location at a time. When moving the measurement location, in the axial direction, Bourne and Sharma [155] and Musil [156] reported that the predicted just-suspension speed remained the same. However, this was not the case for Musil and Vlk [157]. This inconsistency is most likely due to the inaccurate assumption of axial uniformity. Therefore, in the mixing index being proposed in the current study, axial and radial uniformity were not assumed. Instead, the local particle concentrations throughout the various axial and radial locations were taken into consideration. Specifically, this was achieved by using the normalized average of all of the ring-based particle concentrations measured throughout all tomograph planes. The resulting mixing index definition is as follows:

$$
\text { Suspension Index }=\frac{\overline{X_{R, P}}}{X_{t}}
$$

where $X_{t}$ is the system's true concentration throughout the tank - i.e. 2.16 vol\%, which is equivalent to $5 \mathrm{wt} \%$ for a mixture of glass beads and water. 
This mixing index definition is based on the fact that particles can go undetected on the bottom of the tank. This is outside of the measurement range of the ERT system. As a result, the average of the measured particle concentrations is not always equal to the system's true concentration. This only occurs during perfect mixing. When the particles are completely suspended and uniformly distributed. During incomplete suspension, however, particles collect at the bottom of the tank. This results in an average measured concentration that is less than the system's true concentration. Such that, it will even be zero in the case of absolutely no suspension. Therefore, complete suspension will be represented by a suspension index of one. Whereas, no suspension will be represented by a suspension index of zero. The suspension index will then increase with better suspension.

In order to remain consistent with the defined suspension index, the dispersion index used in this study will be redefined as the complement of its original formula. This will result in the index increasing with better performance. This approach was previously used by Hosseini et al. [20]. The resulting numerical definition for the dispersion index used in this study is as follows:

$$
\text { Disperion Index }=1-\frac{1}{\overline{X_{R, P}}} \sqrt{\frac{\sum_{1}^{R} \sum_{1}^{P}\left(X_{R, P}-\overline{X_{R, P}}\right)^{2}}{R P-1}}
$$

Based on this definition, perfect dispersion will be represented with a value of one. Whereas, poor dispersion will be represented with decreasing values such that they can even be negative in cases of very poor dispersion. This is possible since the standard 
deviation of the particle concentrations will exceed the average particle concentration in cases of very poor dispersion.

\subsection{Experimental Results}

Using the proposed mixing index definitions, the experimental data was evaluated as illustrated in Figure 7. These results were obtained by time-averaging the suspension and dispersion indexes - as opposed to the particle concentrations - for 30 frames, after steady-state was reached. These trends qualitatively match experimental observations. The degree of suspension and dispersion increased as the impeller speed was increased. In the later chapters of this thesis, the simulation results will only be evaluated against the experimental data obtained at 550 RPM (i.e. the highlighted data points in Figure 7). This impeller speed was selected to minimize the computational runtime since higher impeller speeds require less time to reach steady-state. The experimental suspension and dispersion indexes occurring at this impeller speed are 0.947 and 0.693 , respectively.

In the experiments, each trial was repeated in triplicate in order to ensure the repeatability of the experiments. The relative standard deviation of the suspension indexes ranged from $0.4 \%$ to $13.3 \%$. These values are relatively unbiased in regards to the impeller speeds. Alternatively, the relative standard deviation of the dispersion indexes ranged from $4 \%$ to $261 \%$ - and were found to be biased in regards to the impeller speeds. In this regard, the relative standard deviation increased with decreasing impeller

speeds. This can be attributed, in part, to the transient manner in which particles would circulate in surges - even at steady-state. During the experiments, the surging behavior 
was observed to occur in cycles such that the surge cycles became slower as the impeller speed was decreased. As a result, the transient behavior at lower impeller speeds could not be captured accurately in a short and fixed measurement time window. In future works, this issue can simply be mitigated by increasing the measurement time window (i.e. the number of tomograph frames captured).

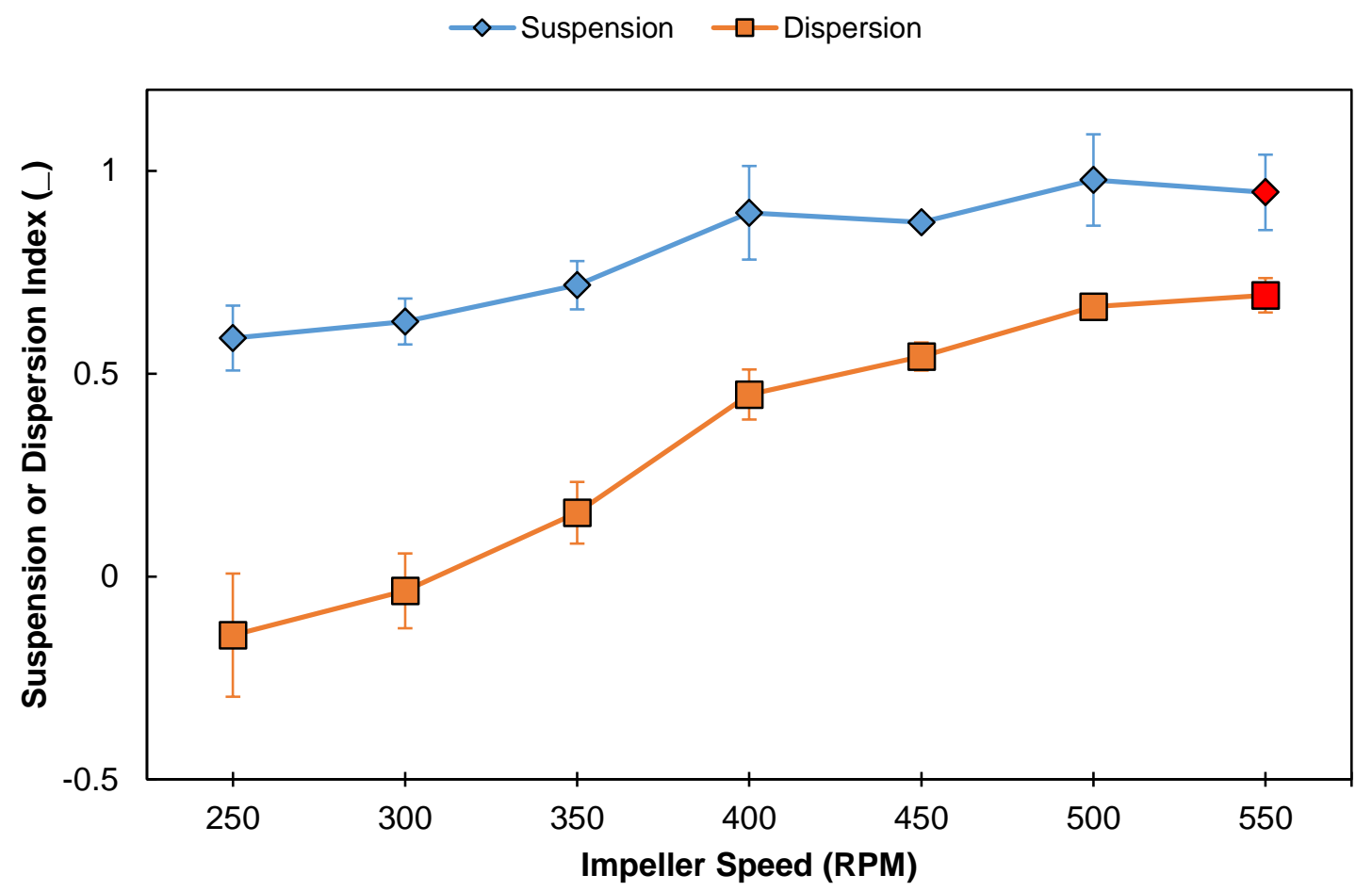

Figure 7. Experimental suspension and dispersion mixing indexes - time-averaged for 30 frames - for varying impeller speeds.

Although, as previously stated, only the experimental data obtained at 550 RPM will be used to evaluate the simulation results. At this impeller speed, the relative standard deviations for the suspension and dispersion indexes are $10 \%$ and $6 \%$ respectively; which both are within an acceptable margin. 


\section{Chapter 4 : Computational Methodologies}

In this chapter, the model development for the current study is outlined. Specifically, this involves detailing the governing equations and fluid-particle interaction force models. Moreover, additional considerations associated with the numerical methods, CFD mesh, particle generation, and model parameters are also specified in this chapter.

\subsection{CFD-DEM Coupled Model}

The two-way coupled CFD-DEM approach is an Eulerian-Lagrangian method that combines the CFD and DEM methodologies. The resulting governing equations will be discussed in the following sub-sections. 


\subsubsection{Governing Equations for Liquid Phase}

The fluid continuity and momentum equations are represented by form $\mathrm{A}$ of the incompressible volume-averaged Navier-Stokes (VANS) equations [88]. Refer to Equation ( 10 ) for the numerical definitions of these equations.

In this work, the flow of the liquid phase is turbulent given the operating range of impeller speeds. Therefore, the realizable $k-\varepsilon$ model was selected in this study for its versatility and superior performance [73]. This model is given by the following equations in tensor format such that $j$ represents the tensor [76]:

$$
\begin{aligned}
& \frac{\partial}{\partial t}\left(\varepsilon_{f} \rho_{f} k\right)+\frac{\partial}{\partial x_{j}}\left(\varepsilon_{f} \rho_{f} k u_{j}\right)=\cdots \\
& \ldots \frac{\partial}{\partial t}\left[\varepsilon_{f}\left(\mu_{f}+\frac{\rho_{f} C_{\mu} k^{2}}{\sigma_{k} \varepsilon}\right) \frac{\partial k}{\partial x_{j}}\right]+\varepsilon_{f}\left(G_{k}+G_{b}-\rho_{f} \varepsilon-Y_{M}+S_{k}\right) \\
& \frac{\partial}{\partial t}\left(\varepsilon_{f} \rho_{f} \varepsilon\right)+\frac{\partial}{\partial x_{j}}\left(\varepsilon_{f} \rho_{f} \varepsilon u_{j}\right)= \frac{\partial}{\partial t}\left[\varepsilon_{f}\left(\mu_{f}+\frac{\rho_{f} C_{\mu} k^{2}}{\sigma_{k} \varepsilon}\right) \frac{\partial \varepsilon}{\partial x_{j}}\right] \ldots \\
& \ldots+\varepsilon_{\mathrm{f}}\left(\rho_{\mathrm{f}} \mathrm{C}_{1} \mathrm{~S} \varepsilon-\rho_{\mathrm{f}} \mathrm{C}_{2} \frac{\varepsilon^{2}}{\mathrm{k}+\sqrt{\mathrm{v}_{\mathrm{f}} \varepsilon}}+\mathrm{C}_{1 \varepsilon} \frac{\varepsilon}{\mathrm{k}} \mathrm{C}_{3 \varepsilon} \mathrm{G}_{\mathrm{b}}+\mathrm{S}_{\varepsilon}\right)
\end{aligned}
$$

where $u$ is the local fluid velocity, $\rho_{f}$ is the fluid density, $\varepsilon_{f}$ is the local fluid porosity, $k$ is the turbulent kinetic energy, $\varepsilon$ is the turbulent dissipation rate, $v_{\mathrm{f}}$ is the fluid kinematic viscosity, and the rest of the parameters are model constants. The need to account for turbulence can be verified using the modified Reynolds number for mixing tanks [19]:

$$
R e_{N}=\frac{N D^{2} \rho_{f}}{\mu_{f}}
$$


where $N$ is the impeller speed, $D$ is the impeller diameter, $\rho_{f}$ is the fluid density, and $\mu_{f}$ is the fluid dynamic viscosity. In the present study, this number can range from approximately 43,000 to 94,000 . This is well above the minimum limit for turbulence of only 5000 .

\subsubsection{Governing Equations for Solid Phase}

Settling particles have a tendency to accumulate into dense particle structures. As a result, the motion of the solid phase is expected to depend not only on fluid-particle interactions, but also on particle-particle and particle-wall interactions. This was taken into consideration when determining the translational and rotational velocities of the particles. The motion of these particles was governed by Newton's second law of motion [54]. Refer to Equation ( 11 ) for the numerical definitions of these governing equations.

In the model developed in this work, the contact forces and torques acting on the particles are obtained from a force-displacement law based on the soft-sphere contact approach. Specifically, the simplified Hertz-Mindlin force-displacement model was used [158]. In this model, the contact forces are defined as follows:

$$
\begin{gathered}
\boldsymbol{f}_{\boldsymbol{n}}^{\boldsymbol{c}}=-k_{n} \delta_{n} \widehat{\boldsymbol{n}}-\eta_{n} \boldsymbol{v}_{r, \boldsymbol{n}} \\
\boldsymbol{f}_{\boldsymbol{t}}^{\boldsymbol{c}}=-\mathrm{k}_{\mathrm{t}} \delta_{t} \hat{\boldsymbol{t}}-\eta_{t} \boldsymbol{v}_{\boldsymbol{r}, \boldsymbol{t}}
\end{gathered}
$$

where $n$ represents normal, $t$ represent tangential, $\boldsymbol{f}^{c}$ is the contact force, $k$ is the elastic spring stiffness, $\delta$ is the particle overlap (i.e. displacement), $\eta$ is the dissipative viscous 
damping, and $v_{r}$ is the relative velocity. The elastic and dissipative coefficients are based on the properties of the particles and can be defined as follows [59], [159]:

$$
\begin{gathered}
k_{n}=\frac{4}{3} Y_{e q} \sqrt{\mathrm{R}_{\mathrm{eq}} \delta_{n}} \\
k_{t}=8 \mathrm{G}_{\mathrm{eq}} \sqrt{\mathrm{R}_{\mathrm{eq}} \delta_{n}} \\
\eta_{n}=-\sqrt{5} \frac{\ln e_{r}}{\sqrt{\ln ^{2} e_{r}+\pi^{2}}} \sqrt{m_{e q} k_{n}} \\
\eta_{t}=-\sqrt{\frac{10}{3}} \frac{\ln e_{r}}{\sqrt{\ln ^{2} e_{r}+\pi^{2}}} \sqrt{m_{e q} k_{t}}
\end{gathered}
$$

where eq represents an equivalent term, $Y$ is the Young's modulus, $G$ is the Shear modulus, $\mathrm{R}$ is the particle radius, $m$ is the particle mass, and $e_{r}$ is the coefficient of restitution. The equivalent terms are functions of the contacting particle properties:

$$
\begin{gathered}
Y_{e q}=\frac{Y_{i} Y_{j}}{Y_{i}\left(1-v_{j}^{2}\right)+Y_{j}\left(1-v_{i}^{2}\right)} \\
\mathrm{G}_{\mathrm{eq}}=\frac{G_{i} G_{j}}{G_{i}\left(2-v_{j}\right)+G_{j}\left(2-v_{i}\right)} \\
R_{e q}=\frac{R_{i} R_{j}}{R_{i}+R_{j}} \\
m_{e q}=\frac{m_{i} m_{j}}{m_{i}+m_{j}}
\end{gathered}
$$

where $v$ is the Poisson ratio. In order to account for the gross stick-slip behavior of contacting particles, the tangential forces are limited by Coulomb's law of friction: 


$$
\left|\boldsymbol{f}_{\boldsymbol{t}}\right| \leq \mu_{s}\left|\boldsymbol{f}_{\boldsymbol{n}}\right|
$$

where $\mu_{s}$ is the sliding friction coefficient. The total contact torques are then determined from the contact forces as expressed in the following equations:

$$
\begin{gathered}
\boldsymbol{M}_{\boldsymbol{r}}=-\mu_{\boldsymbol{r}}\left|\mathbf{f}_{\boldsymbol{n}}\right| R_{c} \widehat{\boldsymbol{\omega}_{\boldsymbol{r}}} \\
\boldsymbol{M}_{\boldsymbol{t}}=R_{c} \widehat{\boldsymbol{v}_{\boldsymbol{n}}} \times \boldsymbol{f}_{\boldsymbol{t}}
\end{gathered}
$$

where $\boldsymbol{M}_{\boldsymbol{r}}$ is the rolling resistance torque, $\boldsymbol{M}_{\boldsymbol{t}}$ is the tangential torque, $\mu_{r}$ is the rolling friction coefficient, and $R_{c}$ is the distance from the particle center to the contact plane.

\subsubsection{Solid-Liquid Interaction Force Models}

The fluid-particle interaction terms, included in the fluid and solid phase governing equations, can account for various forces. This can be illustrated in the numerical definition of these terms [88] - refer to Equation ( 12 ). In the current work, a variety of drag force models will be used to evaluate their capability to accurately reproduce the experimental results in this application. Specifically, drag models that are commonly recommended for dense particle suspensions will be used. These include the Di Felice [94], Gidaspow [87], and Syamlal O'Brien [95] drag models. Refer to Table 2 for the numerical definitions of these drag models. Additionally, other fluid-particle interaction forces are included and excluded in a variety of simulations in order to evaluate their significance. These forces include pressure gradient, virtual mass, Basset, Saffman lift, 
and Magnus lift. Refer to Table 3 for the numerical definitions of these fluid-particle interaction forces.

It should be noted that the Basset force is normally not taken into consideration due to its computational intensity. It is far more difficult to calculate than other fluid-particle interaction forces. In fact, it is computationally more complex and requires calculations that span the entire history of each particle [160], [161]. For this reason, the present work will evaluate this force using the simplified numerical method proposed by van Hinsberg et al. [162]:

$$
\begin{gathered}
\boldsymbol{F}_{\text {Bass }}=\boldsymbol{F}_{\text {Win }}+\boldsymbol{F}_{\text {Tail }} \\
\boldsymbol{F}_{\text {Win }}=\frac{3}{2} d_{p}^{2} \sqrt{\pi \rho_{f} \mu_{f} \Delta t} \boldsymbol{g}_{\text {Win }} \\
\boldsymbol{g}_{\text {Win }}=\frac{4}{3} \boldsymbol{g}_{\mathbf{0}}+\boldsymbol{g}_{\boldsymbol{N}} \frac{N-\frac{4}{3}}{(N-1) \sqrt{N-1}+\left(N-\frac{3}{2}\right) \sqrt{N}} \ldots \\
\ldots+\sum_{n-1}^{N-1} \boldsymbol{g}_{\boldsymbol{n}}\left(\frac{n+\frac{4}{3}}{(n+1) \sqrt{n+1}+\left(n+\frac{3}{2}\right) \sqrt{n}}+\frac{n-\frac{4}{3}}{(n-1) \sqrt{n-1}+\left(n-\frac{3}{2}\right) \sqrt{n}}\right) \\
\boldsymbol{g}(t)=\frac{d \boldsymbol{f}(t)}{d t} \\
\boldsymbol{f}(t)=\boldsymbol{u}-\boldsymbol{v}+\frac{1}{24} d_{p}^{2} \nabla^{2} \boldsymbol{u} \\
\boldsymbol{F}_{\boldsymbol{i}, \boldsymbol{d i}}=3 d_{p}^{2} \sqrt{\pi \rho_{f} \mu_{f} e t_{i}} \exp \left(-\frac{t_{\text {win }}}{2 t_{\mathrm{i}}}\right) \ldots \\
\ldots \times\left\{\boldsymbol{g}_{\boldsymbol{N}}^{m}\left[1-\varphi\left(-\frac{\Delta t}{2 t_{i}}\right)\right]+\boldsymbol{g}_{\boldsymbol{N}+\mathbf{1}} \exp \left(-\frac{\Delta t}{2 t_{i}}\right)\left[\varphi\left(\frac{\Delta t}{2 t_{i}}\right)-1\right]\right\}
\end{gathered}
$$




$$
\begin{gathered}
\varphi(z)=\frac{\exp (z)-1}{z} \\
\boldsymbol{F}_{\boldsymbol{i}, \boldsymbol{r e}}=\exp \left(-\frac{\Delta t}{2 t_{i}}\right) \boldsymbol{F}_{\boldsymbol{i}(\boldsymbol{t}-\Delta \boldsymbol{t})}
\end{gathered}
$$

Van Hinsberg et al. reported that this method decreased both computational costs and error by more than an order of magnitude.

Additionally, it should be reminded that not all fluid-particle interaction forces are taken into consideration explicitly. In essence, they are not always accounted for through fluid-particle interaction force models. Rather, some forces can be accounted for implicitly, through the modification of fluid and particle properties. This is the case for the lubrication force. In literature, it has also been taken into consideration by modifying the particle contact force model or coefficient of restitution [97]. The latter will be demonstrated in the sensitivity test performed in Section 5.2.3 on the global coefficient of restitution. However, this is only a crude approximation. The lubrication force is more accurately accounted for by modifying each individual particles' coefficient of restitution based on their respective Stokes numbers [125], [129]. However, this will come at a higher computational cost.

\subsection{Simulation Conditions and Settings}

In the present work, ANSYS Fluent v16.2 was used as a CFD solver and EDEM v2.7/v2017 was used as a DEM solver. The CFD solver used the iterative SIMPLE scheme for pressure-velocity coupling, a bounded second-order implicit scheme for time integration, a second-order upwind spatial discretization scheme for the interpolation of 
momentum and the turbulent parameters. Such that, integration was performed with a maximum residual of $10^{-3}$. In the DEM solver, the time step was determined based on the Rayleigh time step [63]. Refer to Section 2.4.1.1 for the numerical definition. A percentage of approximately $37 \%$ of the Rayleigh time step was used as the DEM time step. The time steps for the CFD solver and coupling interface were then determined to be 50 times the DEM time step. The simulation parameters, discussed above, are summarized in Table 7.

In order to generally evaluate this model, a case study will be initially evaluated in Section 5.1. This case will consist of using the O'Brien drag model; the pressure gradient, virtual mass, and Saffman lift forces; and the recommended particle parameters listed in Table 7. These particle properties were used in previous solid-liquid simulations [92], [103]. However, they are based on the measured properties of dry materials. This is an acceptable first estimation of wet particle properties. However, refer to Section 5.2.3 for a discussion on the potential calibration of these parameters for wet particles. Additionally, refer to Sections 5.2.1 and 5.2.2 for the justification of selection of the drag model and additional fluid-particle interaction forces.

Table 7. Simulation parameters.

\begin{tabular}{|c|c|c|c|}
\hline Parameter & Variable & Value & Unit \\
\hline Particle Diameter & $\mathrm{d}_{\mathrm{p}}$ & 2 & $\mathrm{~mm}$ \\
\hline Particle Density & $\rho_{\mathrm{p}}$ & 2500 & $\mathrm{~kg} \mathrm{~m}^{-3}$ \\
\hline Young's Modulus & $\mathrm{Y}$ & 10 & $\mathrm{MPa}$ \\
\hline Poisson Ratio & $v$ & 0.25 & $\mathrm{~N} / \mathrm{A}$ \\
\hline Coefficient of Restitution & $\mathrm{e}_{\mathrm{r}}$ & 0.9 & $\mathrm{~N} / \mathrm{A}$ \\
\hline
\end{tabular}




\begin{tabular}{|c|c|c|c|}
\hline Coefficient of Static Friction & $\mu_{\mathrm{s}}$ & 0.3 & $\mathrm{~N} / \mathrm{A}$ \\
\hline Coefficient of Rolling Friction & $\mu_{\mathrm{r}}$ & 0.1 & $\mathrm{~N} / \mathrm{A}$ \\
\hline Particle Weight Fraction & $\mathrm{w}_{\mathrm{p}}$ & 5 & $\mathrm{wt} \%$ \\
\hline Particle Number & $\mathrm{N} / \mathrm{A}$ & 62,904 & $\mathrm{~N} / \mathrm{A}$ \\
\hline DEM Time Step & $\tau_{\mathrm{DEM}}$ & $2 \mathrm{e}-5$ & $\mathrm{~s}$ \\
\hline Liquid Density & $\rho_{\mathrm{f}}$ & 998.2 & $\mathrm{~kg} \mathrm{~m}^{-3}$ \\
\hline Liquid Viscosity & $\mu_{\mathrm{f}}$ & 0.001003 & $\mathrm{~kg} \mathrm{~m}^{-1} \mathrm{~s}^{-1}$ \\
\hline CFD and Coupling Time Step & $\tau_{\mathrm{CFD}}$ & 0.001 & $\mathrm{~s}$ \\
\hline Impeller Speed & $\mathrm{N}$ & 550 & $\mathrm{RPM}$ \\
\hline
\end{tabular}

4.3 Fluid-Phase Mesh and Boundary Conditions used in CFD Solver

In the CFD solver, the geometry described in Section 3.1 was discretized into an unstructured mesh consisting of 45,238 tetrahedral conforming elements. This mesh is illustrated in Figure 8. No slip boundary conditions were applied to the walls of the tank, baffle, and impeller components. This is represented as follows [69]:

$$
u_{x}=u_{y}=u_{z}=0 \text { at the surface }
$$

where $u_{x}, u_{y}$, and $u_{z}$ are the fluid velocities in the $x, y$, and $z$ directions, respectively. Alternatively, a symmetry boundary condition was applied to surface of the liquid exposed to the atmosphere. This is shown in the following equation [78]:

$$
\frac{\partial u_{x}}{\partial n}=\frac{\partial u_{y}}{\partial n}=\frac{\partial u_{z}}{\partial n}=0 \text { at the boundary }
$$


where $n$ is the direction normal to the boundary surface. In order to account for the rotating impeller, a moving reference frame (MRF) was also implemented. This essentially rotated the cylindrical zone enclosing the impeller geometry, as depicted in Figure 8, and left the rest of the geometry stationary. The absolute velocity of the fluid inside this rotating zone was then evaluated as follows [73]:

$$
u=u_{r}+\omega_{M R F} \times r_{M R F}
$$

where $\boldsymbol{u}$ is the absolute fluid velocity, $\boldsymbol{u}_{r}$ is the relative fluid velocity, $\boldsymbol{\omega}_{M R F}$ is the angular velocity of the rotating zone, and $r_{M R F}$ is a position vector within the rotating zone.
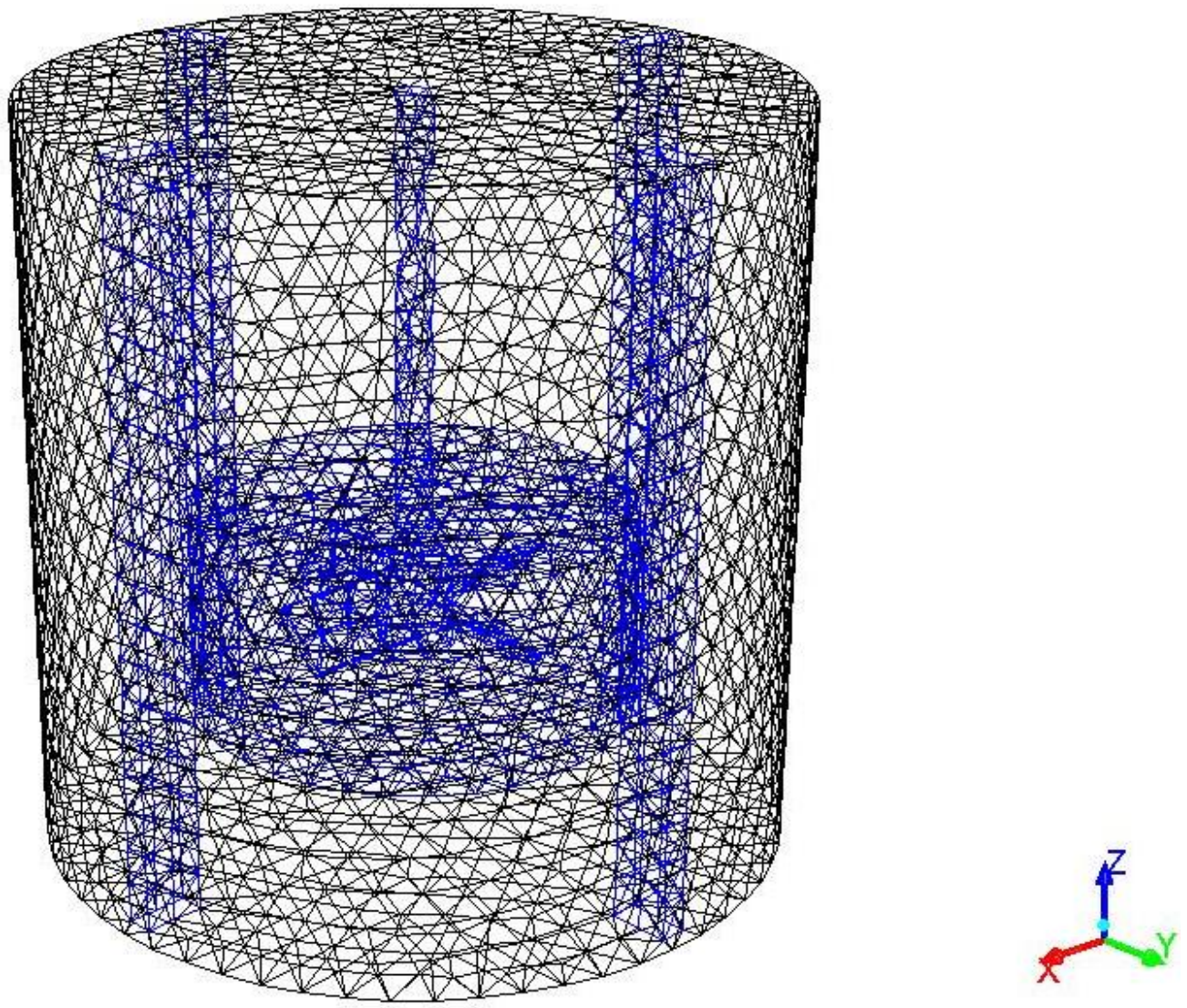

Figure 8. CFD mesh of mixing tank geometry. 


\subsubsection{Mesh Quality in CFD-DEM coupling}

The unresolved CFD-DEM method requires that the mesh size be large compared to the particle diameter but small compared to the computational domain. To meet this requirement, it is recommended that the ratio between the mesh size and particle diameter be greater than 5 [100]. However, this can produce less accurate fluid flow field predictions. This was the case in the present work. The minimum mesh size that could be used to perform stable simulations was still relatively coarse.

\subsubsection{Grid Independence Test in CFD}

By performing further grid refinements on single-phase simulations, it was determined that the current mesh was too coarse to produce accurate fluid flow predictions. The grid refinement was performed by decreasing the mesh size in successive increments. Such that, the number of mesh elements would almost double each time. As this was done, the change in the velocity profile was monitored along a line that was $2.5 \mathrm{~cm}$ below the impeller and parallel with the $\mathrm{x}$-axis. This will be referred to as the monitored line from hereon. The change in the velocity profile was quantified with the normalized root mean square deviation (NRMSD) as follows [163]-[165]:

$$
N R M S D=\frac{1}{\left(u_{\text {ref }, \text { max }}-u_{\text {ref,min }}\right)} \sqrt{\frac{\sum_{k=0}^{N}\left(u_{k}-u_{\text {ref }, k}\right)^{2}}{N}}
$$


where $k$ represents a position along the velocity profile line, $r e f$ represents the reference mesh, and $u$ is the fluid velocity.

Through the further grid refinements, it was determined that the largest gridindependent mesh had 263,507 elements (i.e. 6 times more than the current mesh). When compared to a mesh with 467,528 elements, this mesh produced a NRMSD of $2.5 \%$ and predicted the impeller torque within a $1.5 \%$ accuracy. This is within an acceptable margin of error. Refer to Figure 9 for a comparison between the time-averaged velocity profiles of these meshes. These were obtained by averaging the velocities - at each location along the monitored line - for 1.5 seconds, after steady-state was reached. However, as mentioned previously, a grid independent mesh could not be used in this work since the CFD-DEM simulations became unstable with such a mesh.

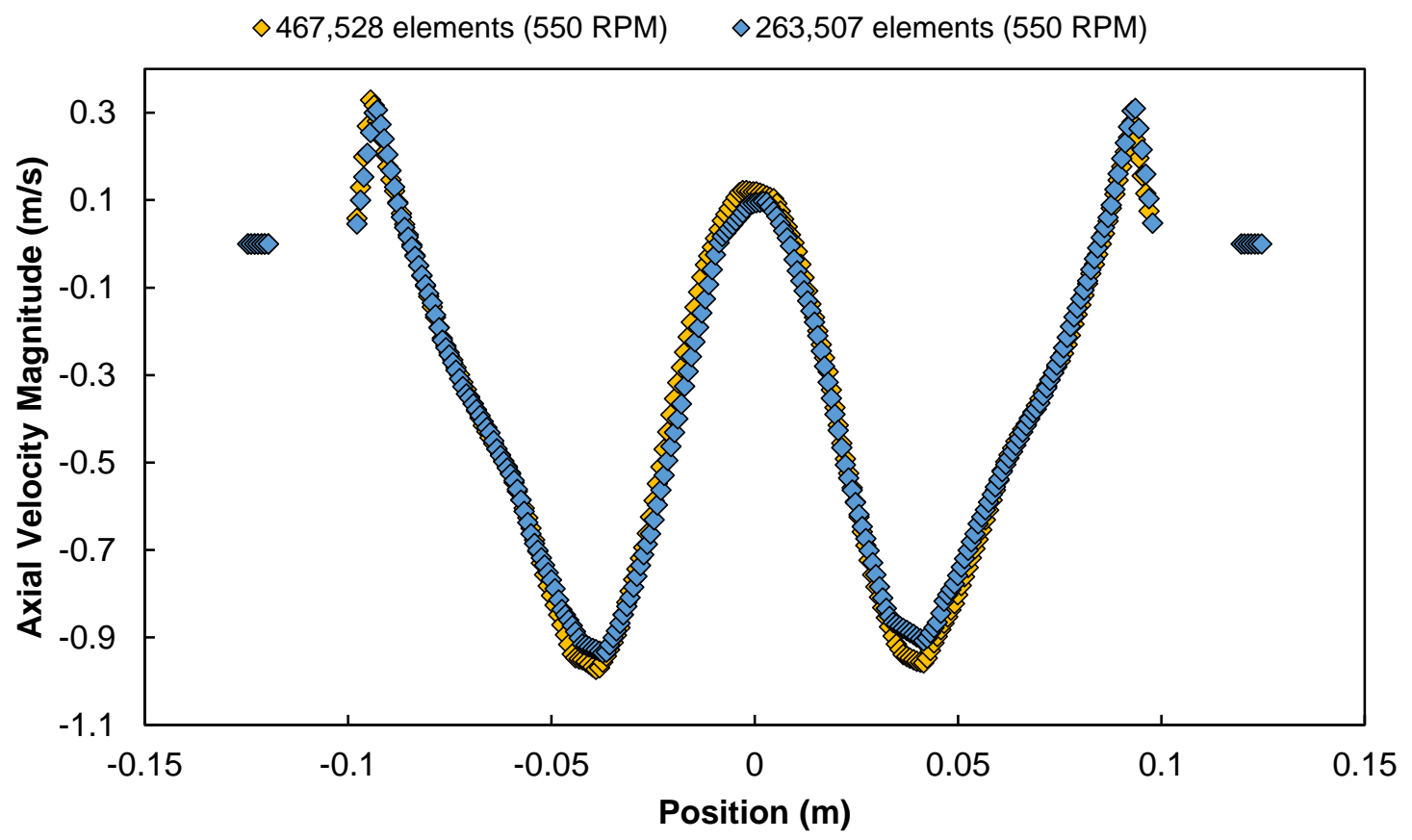

Figure 9. Comparison of time-averaged velocity profiles for grid-independent meshesalong monitored line in liquid-only CFD simulations. 
$\diamond 467,528$ elements (550 RPM) $\diamond 45,238$ elements (550 RPM)

a)

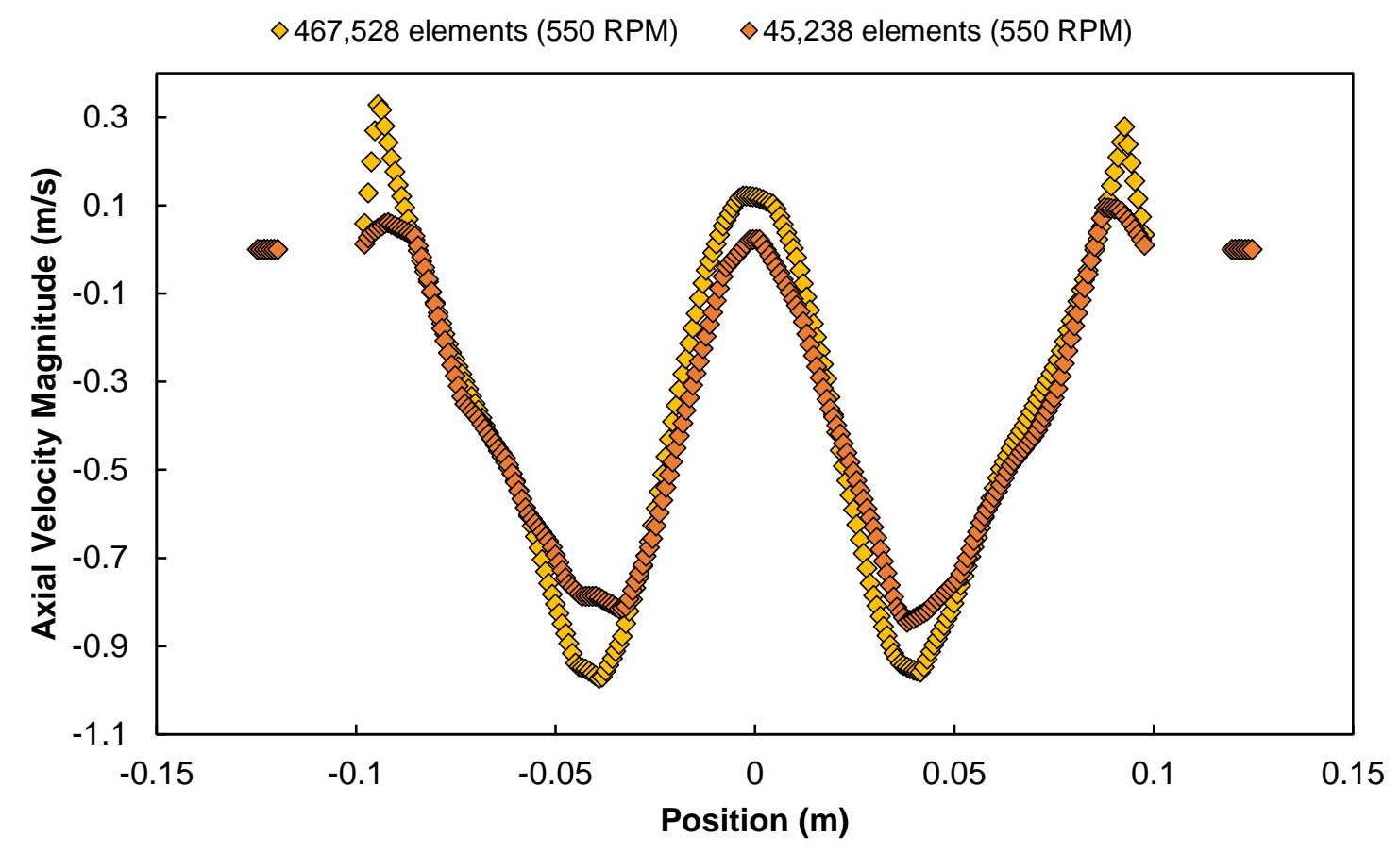

$\diamond 467,528$ elements (550 RPM) $\diamond 45,238$ elements (625 RPM)

b)

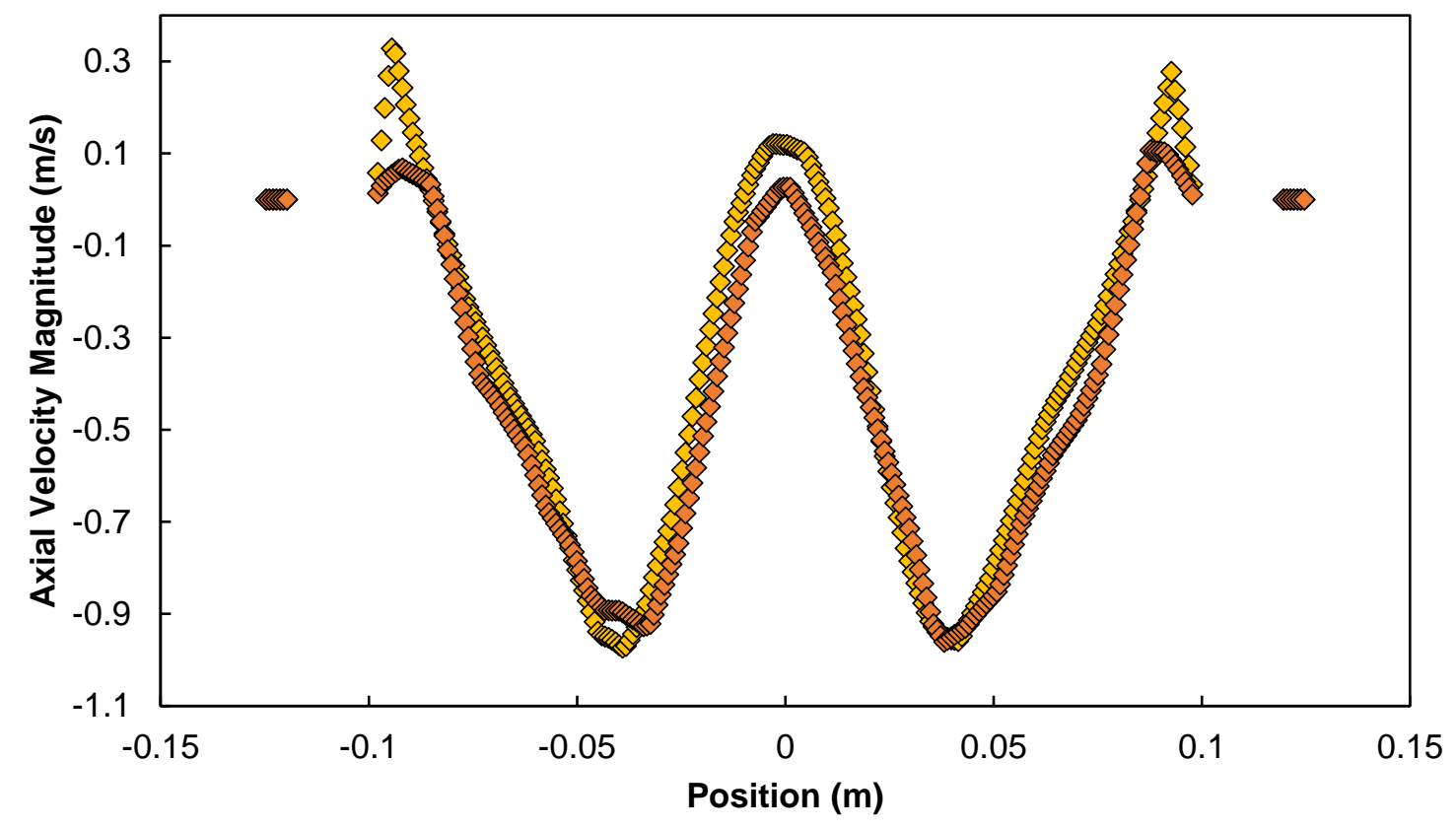

Figure 10. Comparison of time-averaged velocity profiles - along monitored line in liquid-only CFD simulations - for a) current mesh and grid-independent mesh b) current mesh with impeller speed of 625 RPM and grid-independent mesh with impeller speed of 550 RPM. 
Alternatively, when comparing the current mesh with the mesh with 467,528 elements, the NRMSD was $7.2 \%$ and the impeller torque was underestimated by $9.7 \%$. The comparison of the time-averaged velocity profiles of these meshes is illustrated in Figure 10a. As it can be observed, the fluid flow field is significantly underestimated. This will have an adverse effect on the CFD-DEM simulations. In order to evaluate the extent of this error, the impeller speed was increased to better match the expected fluid flow field. In CFD simulations, it was found that, when using the coarse mesh at an impeller speed of 625 RPM, the velocity profile produced was similar to that obtained with the gridindependent mesh and an impeller speed of 550 RPM. Refer to Figure 10b for a comparison between these time-averaged velocity profiles. In the CFD-DEM simulations, the error associated with the coarse mesh could then be quantified by comparing the simulations results of the original simulation to that of the simulation with an impeller speed of 625 RPM. This comparison is detailed in Section 5.1.

\subsection{Particle Generation Procedure}

In order to promote further stability, the particles were introduced into the system only after the liquid flow field had been properly developed. This was accomplished by simulating liquid-only flow for the first 5 seconds of each simulation. The particles were then introduced into the system. All simulations were performed using the same weight fraction of particles, $5 \mathrm{wt} \%$, which is equivalent to 62,904 particles. These particles were introduced over the course of an additional second by randomly generating them at the top of the liquid domain. This is illustrated in Figure 11. Although, it should be noted that 
this procedure was not consistent with the experimental conditions. In essence, experiments were initiated with the particles settled on the bottom of the tank. However, this inconsistency should not have influenced the steady-state conditions. This was the focus of the current work - and therefore, this procedure was considered to be valid.

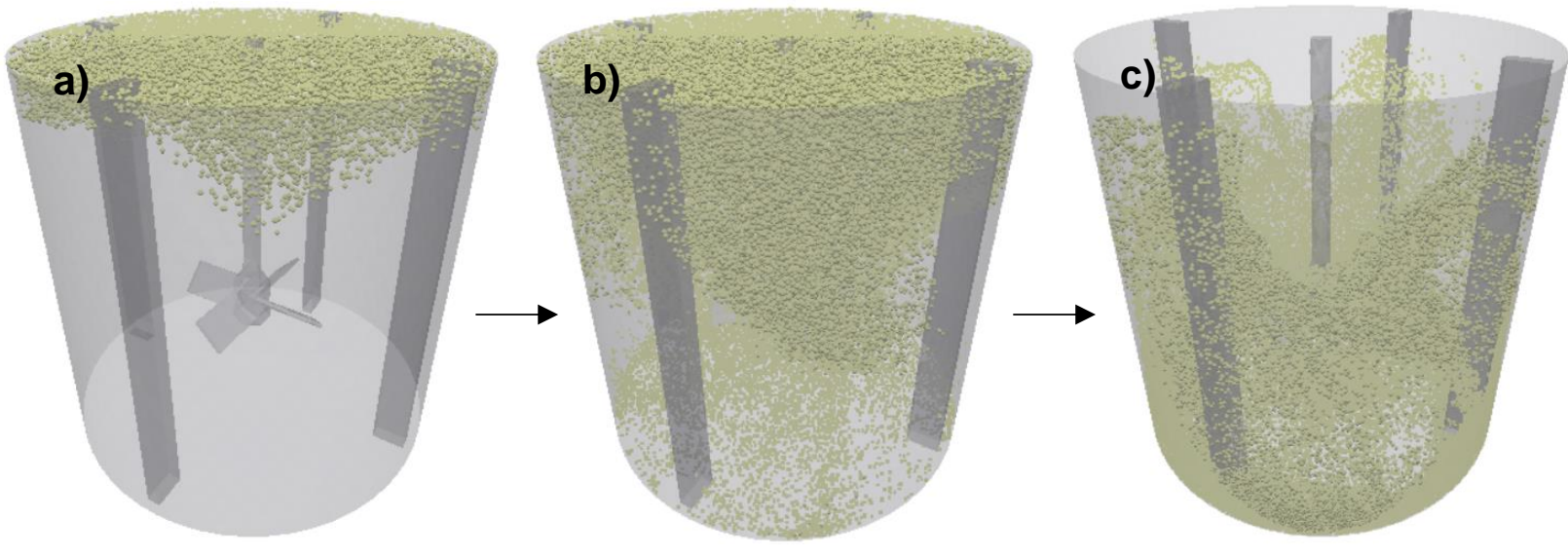

Figure 11. Particle generation images at a) a time shortly after $5 s$ b) a time between 5 and $6 s-$ but closer to $6 s-$ and c) a time long after $6 s$.

The particle generation location was selected in an attempt to reduce the simulation time required to reach steady-state. By moving the particle generation surface, it was found that the surface location did not influence the resulting steady-state properties. Rather, it only influenced the amount of time it took to reach steady-state. Such that, the required simulation time would increase as the surface was lowered into the tank. The simulations were run until the suspension and dispersion index values remained relatively constant. This took an additional 3 to 12 seconds of simulated time depending on the model parameters being used. The steady-state mixing indexes were then evaluated based on the average of the stabilized values. 


\section{Chapter 5 : Results and Discussion}

In order to evaluate the model, detailed in the Chapter 4, the simulation results are compared to experimental results obtained using ERT. Such that, the particle concentration distributions are compared both locally and globally. After evaluating the model, it is then used to evaluate the sensitivity of the predicted mixing indexes to changes in various model parameter. These include the type of drag force model, the inclusion or exclusion of other fluid-particle interaction forces, and the particle contact parameters.

\subsection{Model Evaluation}

When performing simulations, using the model detailed in Chapter 4 , the fluid flow field was confirmed to be downward pumping. This moved the settled particles on the bottom of the tank. Such that, they would accumulate at the center and around the tank 
walls. Some of these settled particles would then become suspended by the upward fluid flow around the tank wall. The suspended particles would then re-circulate back down to the bottom of the tank or accumulate in the dense particle suspension at the tank walls. This is shown in the instantaneous particle suspension pattern depicted in Figure 12 taken after steady-state was reached - as well as in the time-averaged fluid flow field and particle volume fraction plane depicted in Figure 13.

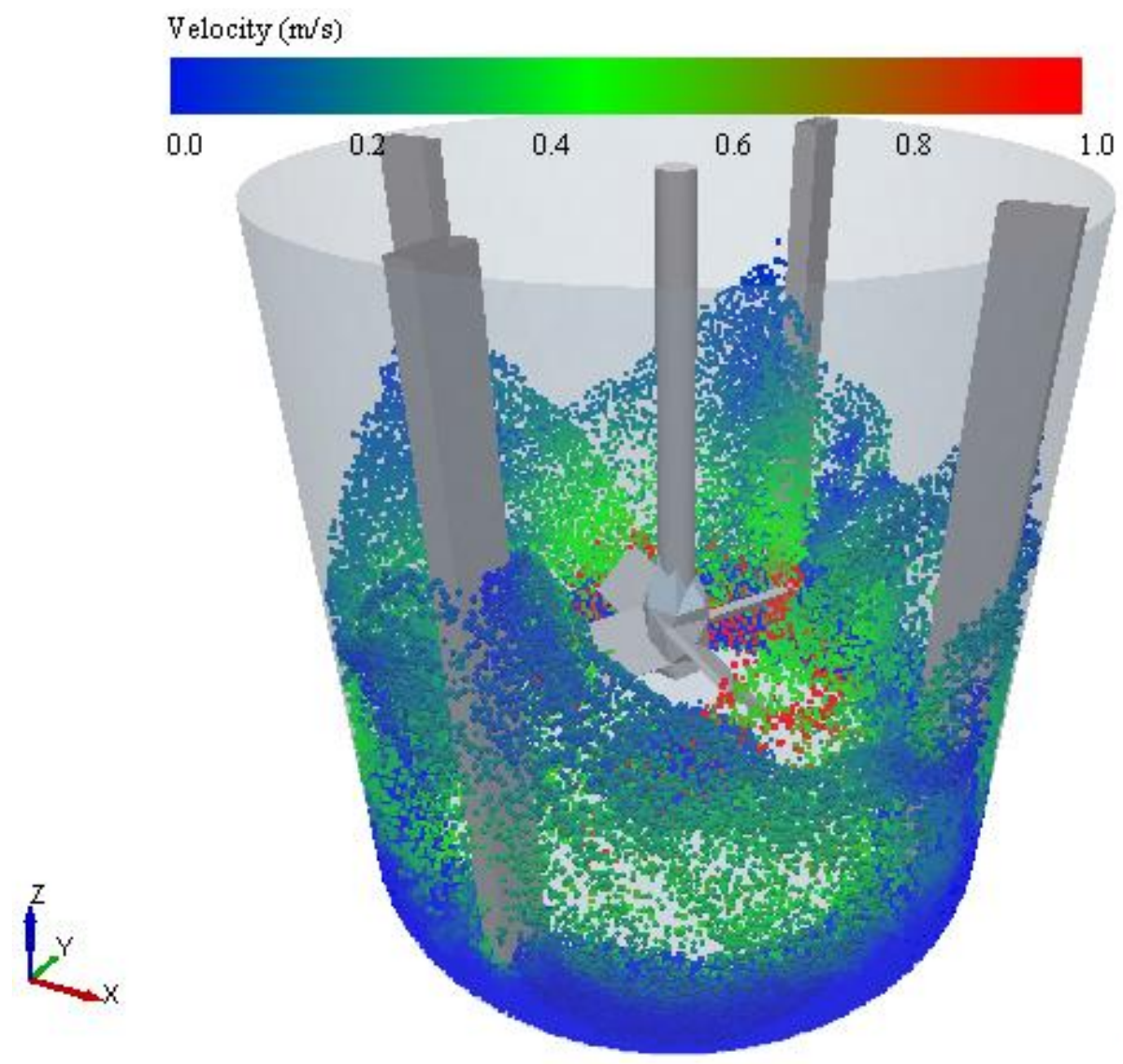

Figure 12. Simulation images of instantaneous particle suspension pattern - taken after steady-state was reached. 
a)

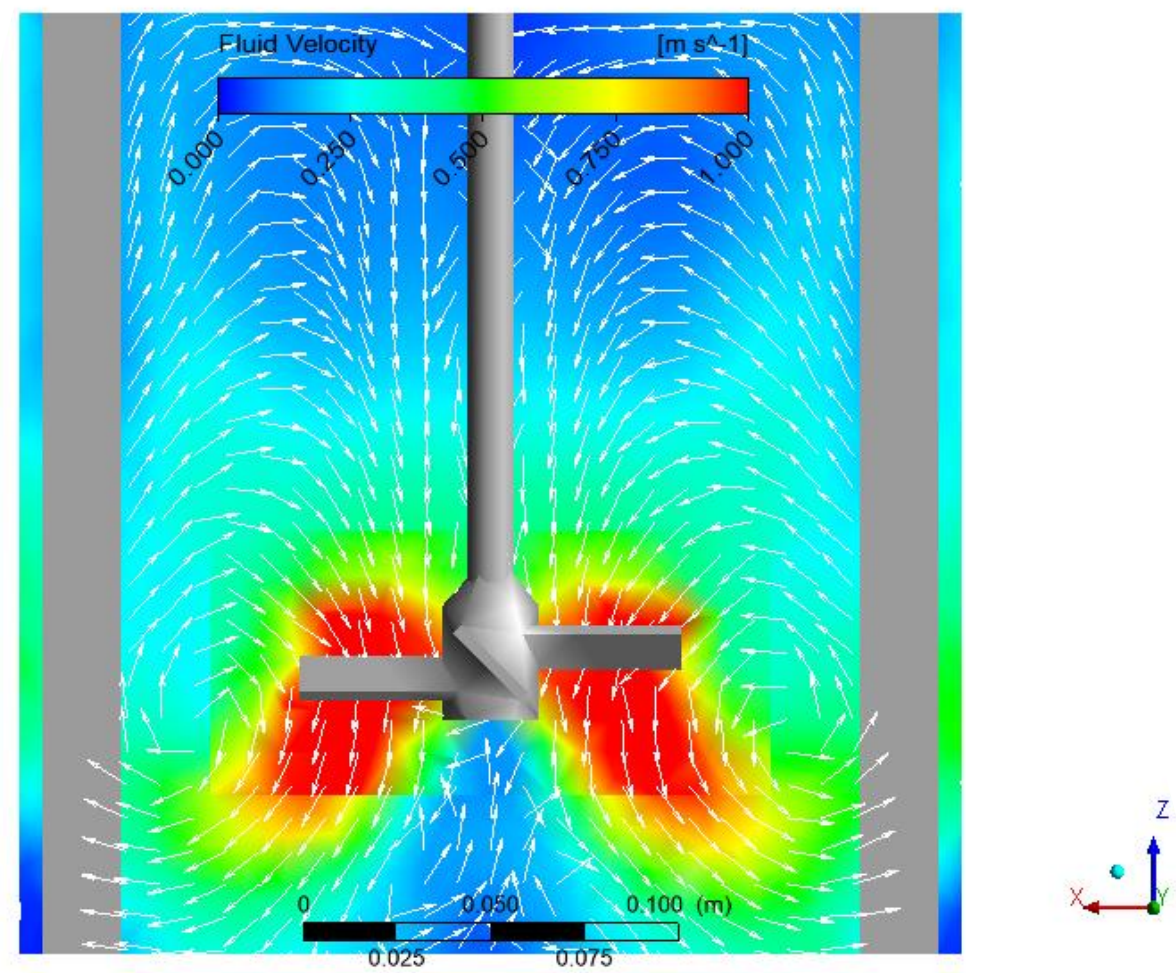

b)
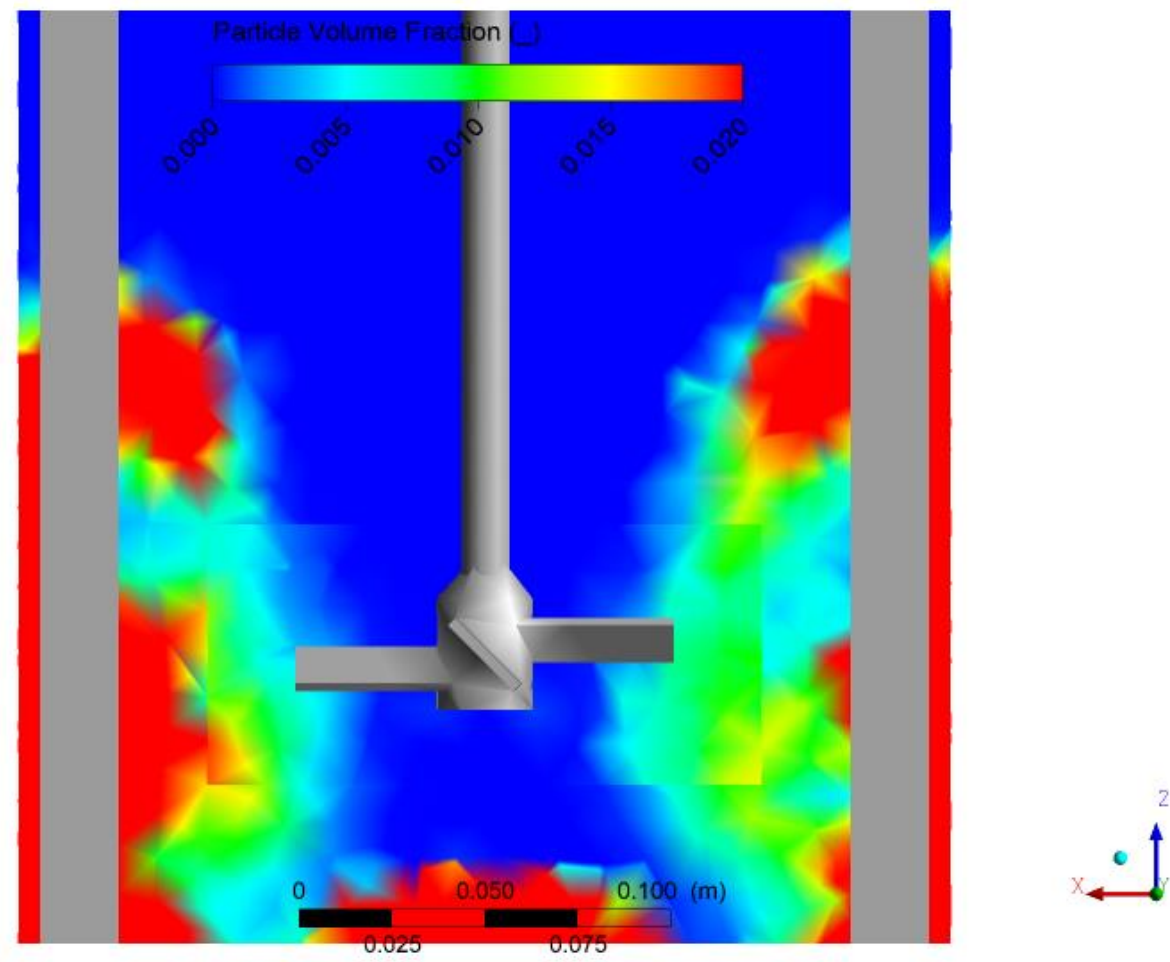

Figure 13. Simulation images of a) time-averaged fluid flow field and b) axial profile of time-averaged particle volume fraction at impeller speed of 550 RPM. 
When evaluating these results, the simulation and experimental particle concentration distributions were compared in two ways. First, by comparing the locallyaveraged ring concentrations to better illustrate the mixing patterns occurring within the tank. Second, by comparing the mixing indexes for convenience. In the former comparison, the ERT tomographs depicted in Figure 5b were locally averaged based on the zoning scheme illustrated in Figure 6 . These results were then compared to the simulation results. When post-processing the simulation results, four planes were defined in a similar manner to that used for the experimental set-up. Each plane was divided into 6 concentric rings as depicted in the zoning scheme illustrated in Figure 14a, which also shows the simulation planes. The resulting comparison between the experimental and simulation results is illustrated in Figure 14b. Such that, each local particle concentration value was scaled relative to the system's true concentration of 2.16 vol\% (i.e. 5 wt $\%$ for a mixture of glass beads and water).

In Figure 14b, it was observed that the general concentration trends were captured in Planes 2, 3, and 4. Although, there were some discrepancies. Specifically, the simulated particle concentrations were too high at the tank walls and almost non-existent towards the center of the tank. However, the majority of the discrepancies were found in Plane 1, which had almost no particles present. This resulted in the underestimation of the simulated cloud height. In essence, the cloud height was found to be between Planes 1 and 2 for the simulations and above Plane 1 for the experiments. 
a)

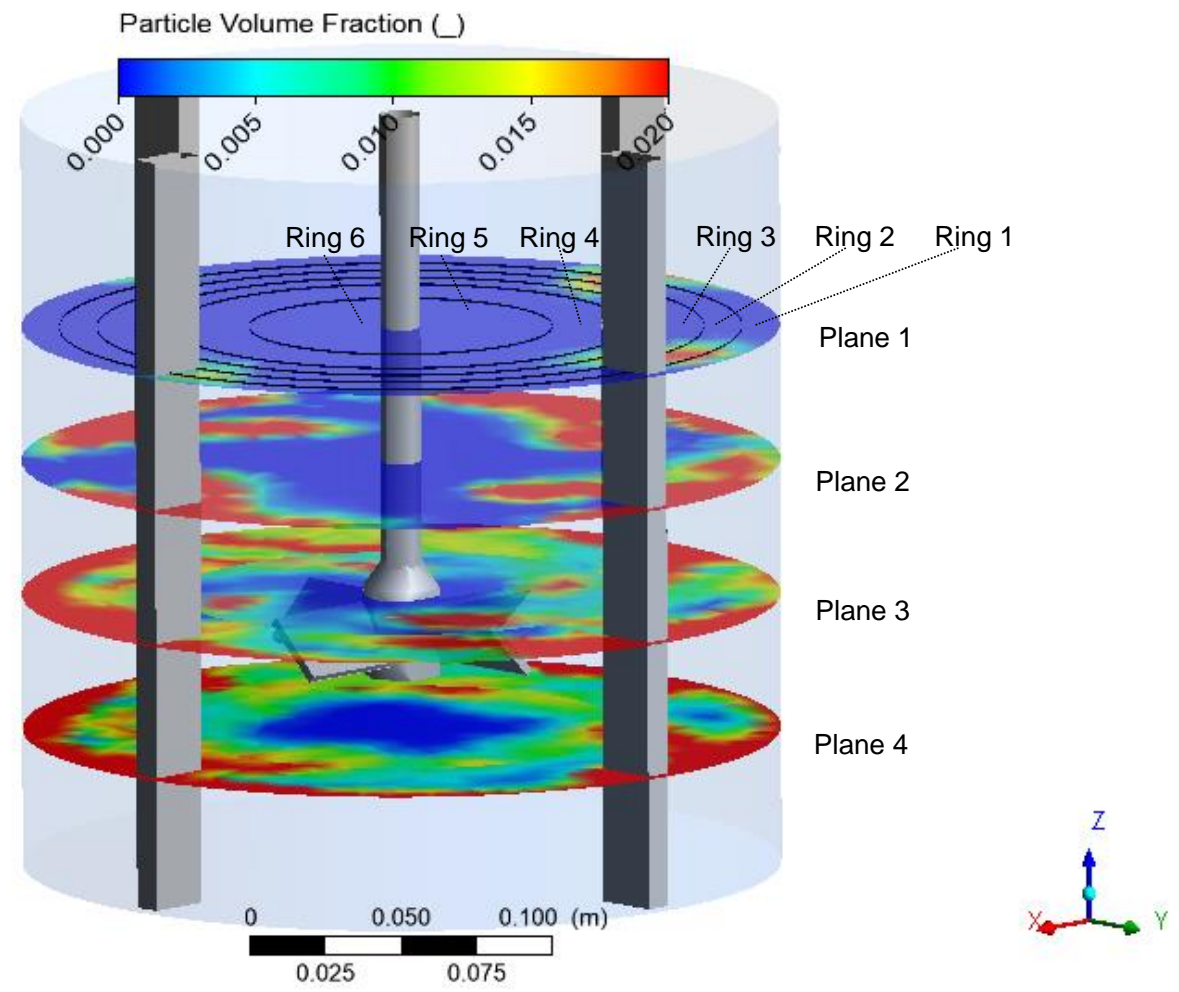

$\diamond$ Experiment $\quad \square$ Simulation - 550 RPM

b)

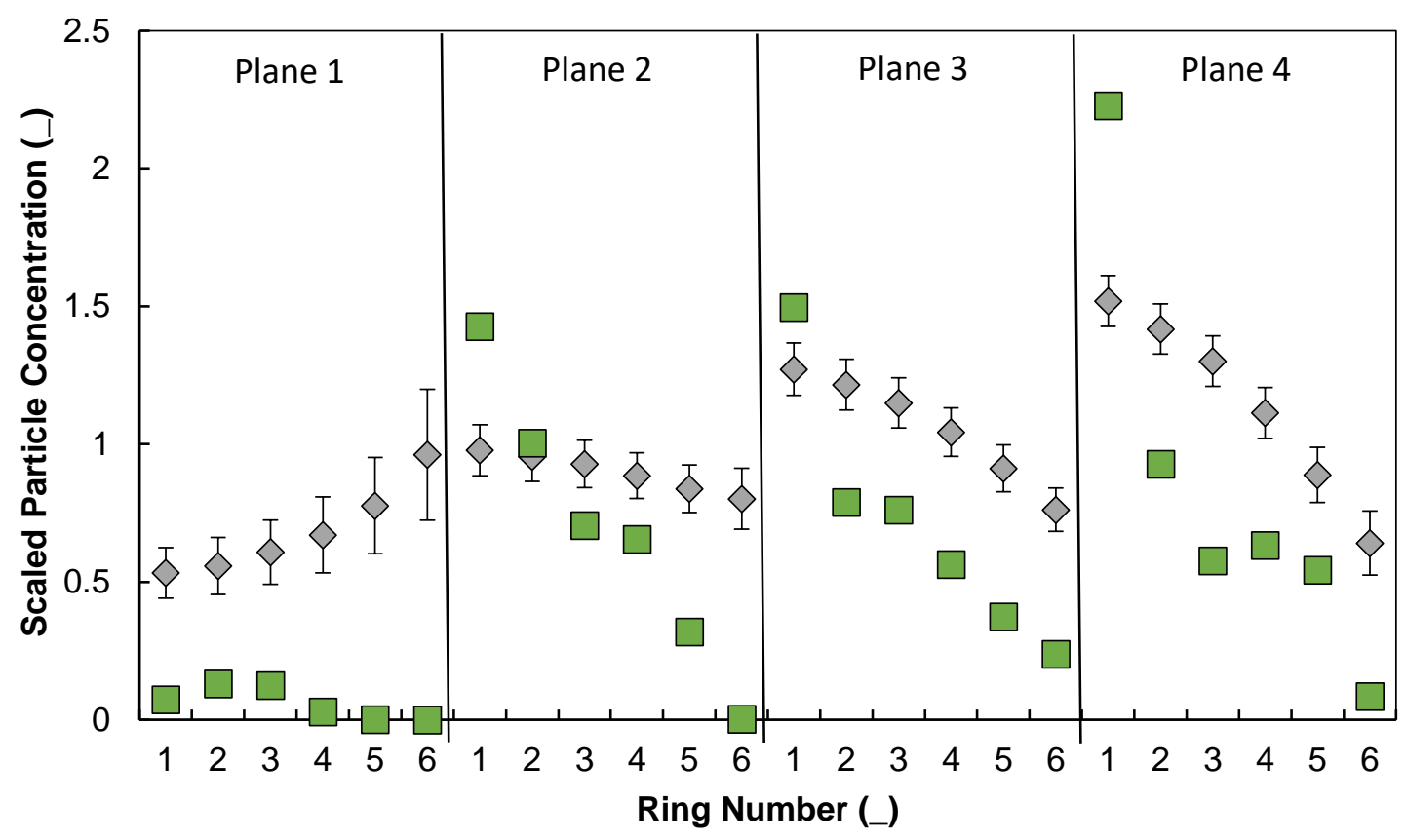

Figure 14: Images of a) simulated time-averaged particle concentration planes and zoning scheme and b) comparison between experiment and simulation time-averaged local particle concentrations at impeller speed of 550 RPM. 
Based on the simulation results, the suspension and dispersion indexes are 0.571 and 0.024 respectively. When comparing these values to the experimental results presented in Section 3.4, the error in the simulation was quantified. This was achieved by first calculating the errors in the suspension and dispersion indexes as the error percentages between the experimental and simulation values. Then, the relative error was determined as the average of those error percentages. This is represented as follows:

$$
\text { Relative Error }=\frac{1}{2}\left(\frac{S I_{\text {exp }}-S I_{\text {sim }}}{S I_{\text {exp }}}+\frac{D I_{\text {exp }}-D I_{\text {sim }}}{D I_{\text {exp }}}\right) \times 100 \%
$$

where $\exp$ represents the experimental values, $\operatorname{sim}$ represents the simulation values, $S I$ is the suspension index, and DI is the dispersion index. For this case study, the relative error was $68.1 \%$. This was calculated from suspension and dispersion index errors of $39.7 \%$ and $96.5 \%$, respectively. It can be observed that the majority of this error was attributed to the deviation in particle dispersion. This confirms that both suspension and dispersion needed to be taken into consideration when evaluating the accuracy of the model.

The relative error for this case study was also compared to those calculated by only considering either the axial or radial distributions. These were determined by taking the complements of the mixing indexes depicted in Equations ( 30 ) and ( 31 ). The resulting relative errors were $45.3 \%$ and $54.9 \%$, respectively. These values are both less than the original relative error that was calculated by considering the overall distribution. 
This proves that both axial and radial distributions needed to be taken into consideration to accurately evaluate mixing quality.

The discrepancies observed in Figure $14 \mathrm{~b}$ can be attributed, in part, to the modeling limitations associated with the CFD-DEM model including (1) the mesh size, (2) the application of the MRF method, and (3) the fluid-particle interaction force models as well as the turbulence closure equations. As mentioned previously, as an inherent limitation associated with the CFD-DEM method, the mesh size must be coarse compared to the particle size to ensure stability. However, in this study, the minimum stable mesh size was still quite coarse. This resulted in the underestimation of the fluid velocities and the fluid-particle interaction forces. The severity of this error can be analyzed by running a stand-alone simulation with the coarse mesh and an artificially increased impeller speed (i.e. 625 RPM). This produced a fluid flow field similar to the flow field obtained using a simulation performed with a grid independent mesh and an impeller speed of 550 RPM. Refer to Section 4.3.2. With the artificially increased impeller speed, it was observed that the simulated cloud height was no longer underestimated as depicted in Figure 15. This resulted in a reduced relative error of $47.7 \%$. This was a result of both the suspension and dispersion index errors being reduced (i.e. to $21.1 \%$ and $74.3 \%$, respectively). This suggests that the mesh size limitation contributed significantly to the error in both particle suspension and dispersion.

The MRF method is not conventionally used in Eulerian-Lagrangian simulations. Rather, it is more commonly applied to Eulerian simulations in which mesh size is not a limiting factor. In those simulations, the mesh could be refined when necessary. More specifically, it could be refined around the interface used for MRF communication. 
However, this is not the case for Eulerian-Lagrangian simulations, which can result in a lack of mesh quality at the MRF interface. If this occurs, the fluid velocities and pressures being communicated across the MRF interface would be subjected to interpolation errors. This would then lead to small differences in the velocity and pressure across the interface. In this study, these differences could have hindered particles from crossing over the interfacial boundary. Specifically, from the tank periphery to the impeller body. This would have then caused the degree of particle dispersion to be underestimated. Therefore, it is recommended that other modeling techniques such as the sliding mesh and immersed boundary methods [102], [103], [152] be investigated in future works in order to mitigate these potential sources of error.

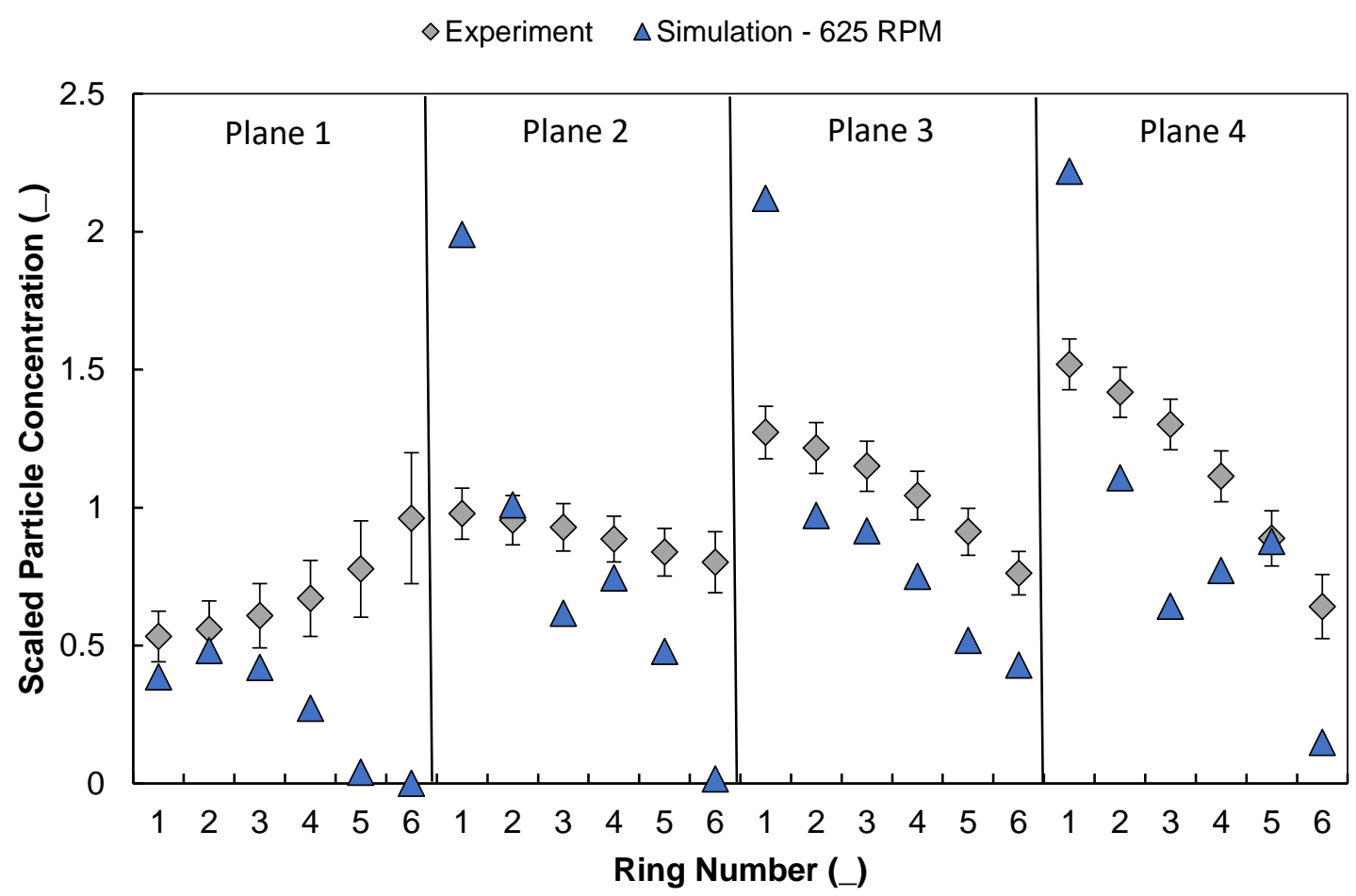

Figure 15: Comparison of time-averaged local particle concentrations with impeller speed of 550 RPM for experiment and 625 RPM for simulation. 
The significant modeling constraints associated with the fluid-particle interaction force models will be discussed later in Section 5.2.2. However, in regards to the turbulence closure equations, current models do not account for turbulence modulation. This occurs as a result of the particulate phase modifying the liquid phase flow patterns. Considering the particle size and concentration used in the current study, neglecting turbulence modulation could have potentially influenced the degree of particle suspension and dispersion. In literature, it has been found to significantly influence predictions in fluid velocities and the hydrodynamic behaviour [166].

Additionally, it should be noted that error, although to a lesser extent, is also attributed to experimental limitations. In this regard, the spatial accuracy of the measurement method used in this study, ERT, was relatively low - with a resolution of only $5 \%$ of the vessel diameter. Furthermore, minor imperfections in the experimental setup would have also contributed to the observed deviation.

Regardless of these limitations, when increasing the impeller speed, the model showed a realistic trend similar to that observed in the experimental results. Refer to Figure 7 for the experimental trends. The simulated trends are illustrated in Figure 16, over a series of impeller speeds ranging from 450 RPM to 800 RPM. In this figure, the simulated suspension and dispersion indexes increase with increasing impeller speeds. Then, plateau at a maximum value, in a similar manner to the experimental trend lines. However, these maximum values were achieved at higher impeller speeds, when compared to the experimental results. Although, the simulated maximum suspension index was even very close to the experimental value. These values were 0.979 and 0.962 respectively. However, the simulated maximum dispersion index was still underestimated 
(i.e. by $70.7 \%$ ). This further confirms that both the particle suspension and dispersion should be used to evaluate this model. If only suspension was taken into consideration, the significant limitation in reproducing dispersion would have not been identified.

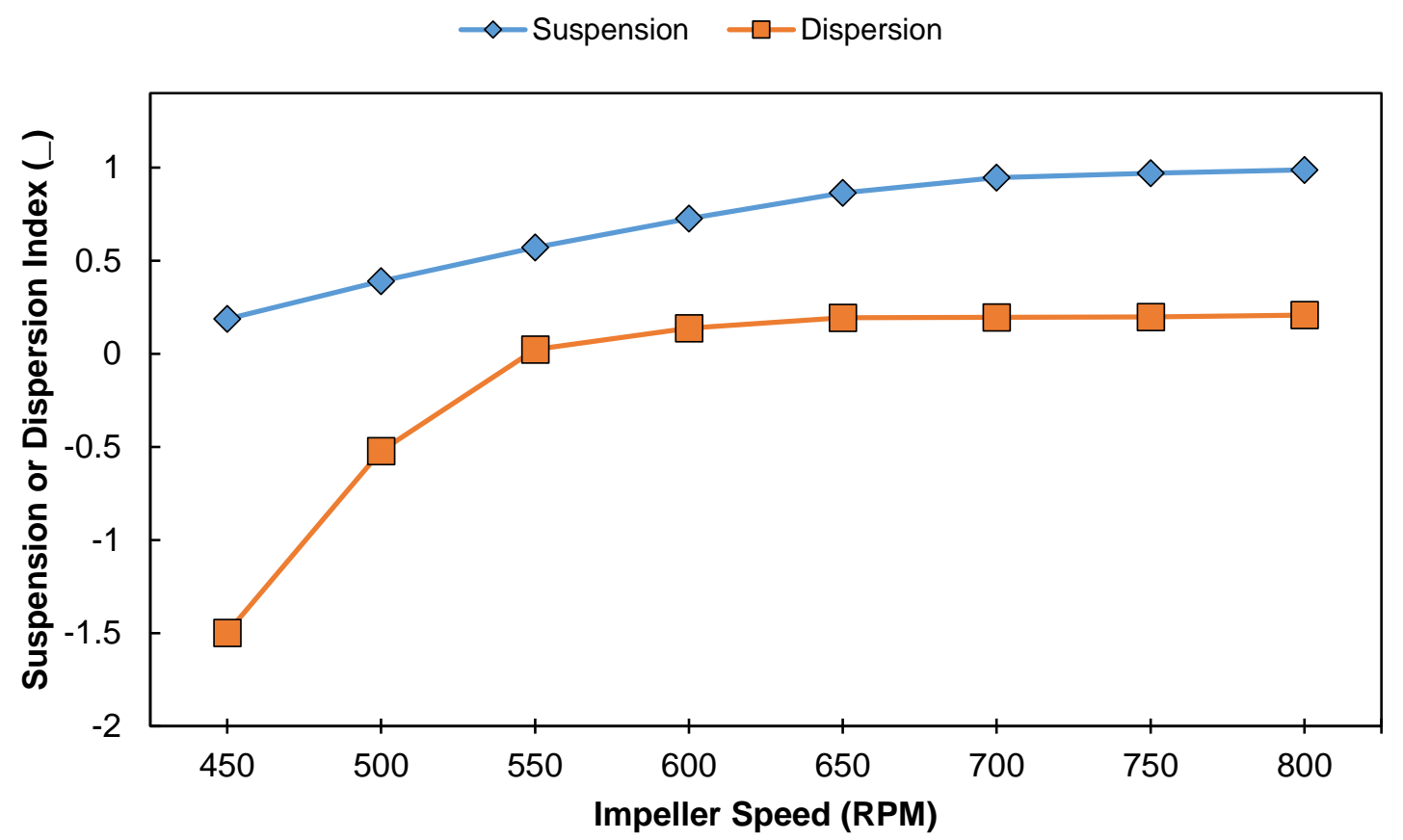

Figure 16. Simulation suspension and dispersion indexes for varying impeller speeds.

\subsection{Model Sensitivity Tests for Mixing Quality}

Next, the sensitivity of the model was investigated by changing various model parameters and evaluated through the resulting changes in the mixing indexes. The model parameters studied include (1) the type of drag force model, (2) the exclusion or inclusion of other fluid-particle interaction forces, and (3) various particle properties. The specific parameters investigated are listed in Table 8 and the results are summarized in Table 9. These will be discussed in the following sub-sections. 
Table 8. Summary of different cases for model sensitivity tests conducted at impeller speed of 550 RPM.

\begin{tabular}{|c|c|c|c|c|c|c|c|c|c|c|}
\hline Case & $\begin{array}{l}\text { Drag } \\
\text { Force } \\
\text { Model }\end{array}$ & $\begin{array}{l}\text { Pressure } \\
\text { Gradient }\end{array}$ & $\begin{array}{l}\text { Virtual } \\
\text { Mass }\end{array}$ & $\begin{array}{c}\text { Saffman } \\
\text { Lift }\end{array}$ & $\begin{array}{c}\text { Magnus } \\
\text { Lift }\end{array}$ & Basset & $\begin{array}{c}\text { Sliding } \\
\text { Friction } \\
\text { (_) }\end{array}$ & $\begin{array}{c}\text { Rolling } \\
\text { Friction } \\
\text { (_) }\end{array}$ & $\begin{array}{c}\text { Young's } \\
\text { Modulus } \\
\text { (MPa) }\end{array}$ & $\begin{array}{c}\text { Restitution } \\
\text { Coefficient } \\
\text { ( })\end{array}$ \\
\hline $1 \mathrm{~A}$ & Di Felice & \multirow[t]{3}{*}{ Yes } & \multirow[t]{3}{*}{ Yes } & \multirow[t]{3}{*}{ Yes } & \multirow[t]{3}{*}{ No } & \multirow[t]{3}{*}{ No } & \multirow[t]{3}{*}{0.3} & \multirow[t]{3}{*}{0.1} & \multirow[t]{3}{*}{10} & \multirow[t]{3}{*}{0.9} \\
\hline 1B & Gidaspow & & & & & & & & & \\
\hline $1 \mathrm{C}$ & O'Brien & & & & & & & & & \\
\hline $2 A$ & \multirow{5}{*}{ O'Brien } & No & Yes & Yes & No & No & \multirow[t]{5}{*}{0.3} & \multirow[t]{5}{*}{0.1} & \multirow[t]{5}{*}{10} & \multirow[t]{5}{*}{0.9} \\
\hline 2B & & Yes & No & Yes & No & No & & & & \\
\hline $2 \mathrm{C}$ & & Yes & Yes & No & No & No & & & & \\
\hline 2D & & Yes & Yes & Yes & Yes & No & & & & \\
\hline $2 \mathrm{E}$ & & Yes & Yes & Yes & No & Yes & & & & \\
\hline $3 A$ & \multirow[t]{9}{*}{ O'Brien } & \multirow[t]{9}{*}{ Yes } & \multirow[t]{9}{*}{ Yes } & \multirow[t]{9}{*}{ Yes } & \multirow[t]{9}{*}{ No } & \multirow[t]{9}{*}{ No } & 0.15 & 0.1 & 10 & 0.9 \\
\hline 3B & & & & & & & 0.9 & 0.1 & 10 & 0.9 \\
\hline $3 C$ & & & & & & & 0.9 & 0.01 & 10 & 0.9 \\
\hline 3D & & & & & & & 0.9 & 0.5 & 10 & 0.9 \\
\hline $3 E$ & & & & & & & 0.9 & 1 & 10 & 0.9 \\
\hline $3 F$ & & & & & & & 0.9 & 0.1 & 1 & 0.9 \\
\hline $3 G$ & & & & & & & 0.9 & 0.1 & 100 & 0.9 \\
\hline $3 \mathrm{H}$ & & & & & & & 0.9 & 0.1 & 10 & 0.01 \\
\hline 31 & & & & & & & 0.9 & 0.1 & 10 & 0.7 \\
\hline
\end{tabular}


Table 9. Case results for sensitivity tests.

\begin{tabular}{|c|c|c|c|c|c|}
\hline Case & $\begin{array}{c}\text { Suspension } \\
\text { Index (_) }\end{array}$ & $\begin{array}{c}\text { Dispersion } \\
\text { Index ( })\end{array}$ & $\begin{array}{c}\text { Suspension } \\
\text { Index Error (\%) }\end{array}$ & $\begin{array}{c}\text { Dispersion } \\
\text { Index Error (\%) }\end{array}$ & $\begin{array}{c}\text { Relative } \\
\text { Error (\%) }\end{array}$ \\
\hline 1A & 0.466 & -0.111 & $50.8 \%$ & $116.1 \%$ & $83.4 \%$ \\
\hline 1B & 0.549 & -0.165 & $42.1 \%$ & $123.8 \%$ & $82.9 \%$ \\
\hline 1C & 0.571 & 0.024 & $39.7 \%$ & $96.5 \%$ & $68.1 \%$ \\
\hline 2A & 0.517 & -0.207 & $45.4 \%$ & $129.9 \%$ & $87.6 \%$ \\
\hline 2B & 0.481 & -0.228 & $49.2 \%$ & $132.9 \%$ & $91.1 \%$ \\
\hline 2C & 0.482 & -0.109 & $49.1 \%$ & $115.7 \%$ & $82.4 \%$ \\
\hline 2D & 0.546 & -0.043 & $42.4 \%$ & $106.2 \%$ & $74.3 \%$ \\
\hline 2E & 0.556 & 0.050 & $41.2 \%$ & $92.9 \%$ & $67.1 \%$ \\
\hline 3A & 0.632 & 0.055 & $33.3 \%$ & $92.0 \%$ & $62.7 \%$ \\
\hline 3B & 0.551 & 0.026 & $41.8 \%$ & $96.2 \%$ & $69.0 \%$ \\
\hline 3C & 0.688 & 0.084 & $27.3 \%$ & $87.8 \%$ & $57.6 \%$ \\
\hline 3D & 0.292 & -0.321 & $69.2 \%$ & $146.2 \%$ & $107.7 \%$ \\
\hline 3E & 0.329 & -0.897 & $65.2 \%$ & $229.4 \%$ & $147.3 \%$ \\
\hline 3F & 0.558 & -0.010 & $41.1 \%$ & $101.5 \%$ & $71.3 \%$ \\
\hline 3G & 0.523 & -0.011 & $44.8 \%$ & $101.6 \%$ & $73.2 \%$ \\
\hline 3H & 0.481 & -0.094 & $49.3 \%$ & $113.6 \%$ & $81.4 \%$ \\
\hline 3I & 0.509 & -0.066 & $46.3 \%$ & $109.5 \%$ & $77.9 \%$ \\
\hline
\end{tabular}

\subsubsection{Influence of Drag Models}

The drag force is the dominant force influencing fluid-particle interactions. For this reason, the drag force model plays a significant role in the accuracy of particle concentration predictions. This will be demonstrated by the following evaluation in which various drag models will be compared. More specifically, three of the drag force models commonly used and recommended for dense particle suspensions will be employed in 
the model. These include the Di Felice [94], Gidaspow [87], and O'Brien [95] models. When comparing these models to each other, two observations were made. First, the greatest suspension index error (i.e. 50.8\%) was obtained from the simulation employing the Di Felice drag model. Second, the dispersion index errors obtained using the Di Felice and Gidaspow models were much greater than the dispersion index error obtained using the O'Brien drag model (i.e. they were $19.6 \%$ to $27.3 \%$ greater). This is illustrated in Figure 17.

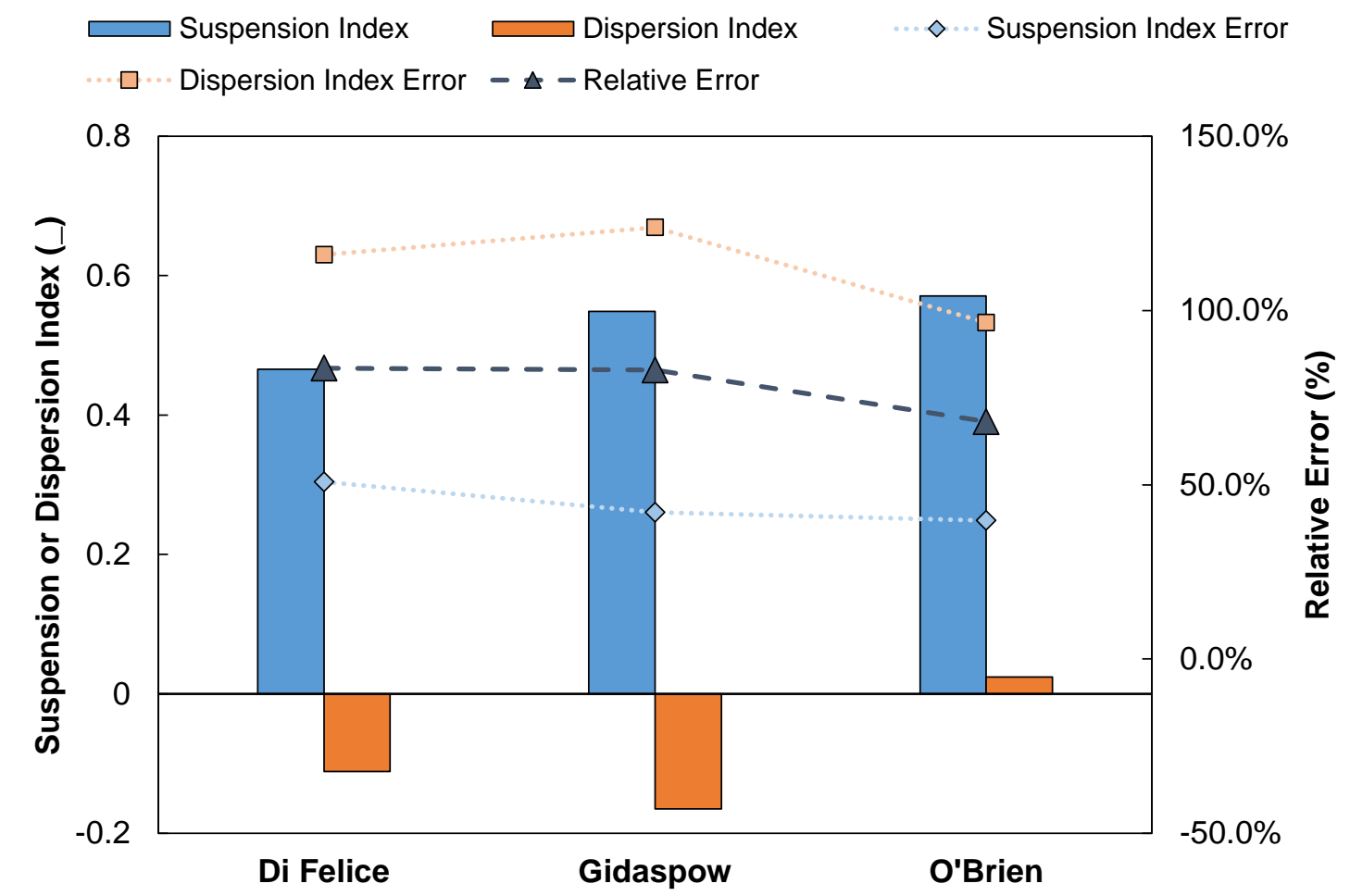

Figure 17. Comparison for the drag force model based on Cases 1A, 1B, and 1C.

These observations can be attributed to the limitations of the drag models. The $\mathrm{Di}$ Felice model is based on an empirical voidage function, which accounts for the influence of neighbouring particles. However, this function was derived under laminar flow 
conditions [51]. Therefore, it is more reliable in the laminar regime. The Gidaspow model, on the other hand, has been successfully used in the turbulent regime. However, this model is normally recommended for when viscous forces are dominant [1], [167]. For the present study, the opposite holds true.

Based on this comparison, the O'Brien model is better suited for this particular application. It had the lowest relative error and best trade-off between the suspension and dispersion index errors. However, it should be noted that this model still produces some discrepancies. Therefore, it is recommended that other drag force models be investigated in futures works to further improve drag force predictions.

\subsubsection{Influence of Other Fluid-particle Interaction Forces}

The fluid-particle interaction forces, other than drag, may have also contributed to the suspension and dispersion of the particles. The pressure gradient, virtual mass, and Basset forces are likely to be significant under the current operating conditions investigated in this study. Specifically, these forces become more significant with lower particle-to-fluid density ratios. In addition to this, the Saffman and Magnus lift forces are also likely to be significant. These forces become more significant with higher rates of shear and particle collisions, which would also be experienced in this application. Therefore, the significance of these forces will be evaluated in the following investigation.

In this assessment, Case $1 \mathrm{C}$ was used as the base case in which the other cases were based upon. Specifically, Cases $2 \mathrm{~A}$ to $2 \mathrm{E}$. In these cases, the fluid-particle interaction forces used in the base case were duplicated with the exception of one force. 
This force would either be excluded if it was originally included or included if it was originally excluded. When doing this, all of the resulting cases could then be compared to the same base case. Such that, the significance of accounting for any of these forces could be evaluated. More specifically, the significance of the forces was evaluated by comparing the relative errors as well as mixing indexes. Such that, the case values associated with excluding the force were subtracted from those associated with including it. This is represented as follows:

Suspension or dispersion index difference $=$ suspension or dispersion index of case including interaction force - suspension or dispersion index of case excluding interaction force

Change in relative error $=$ Relative error of case including interaction force - Relative error of case excluding interaction force

Based on this approach, the change in the relative error would be negative if the inclusion of the interaction force reduces error and positive if it actually introduces more error. Whereas, the change in the mixing indexes would be positive if the inclusion of the interaction force increases mixing quality and negative if it actually decreases mixing quality. This assessment is illustrated in Figure 18. 


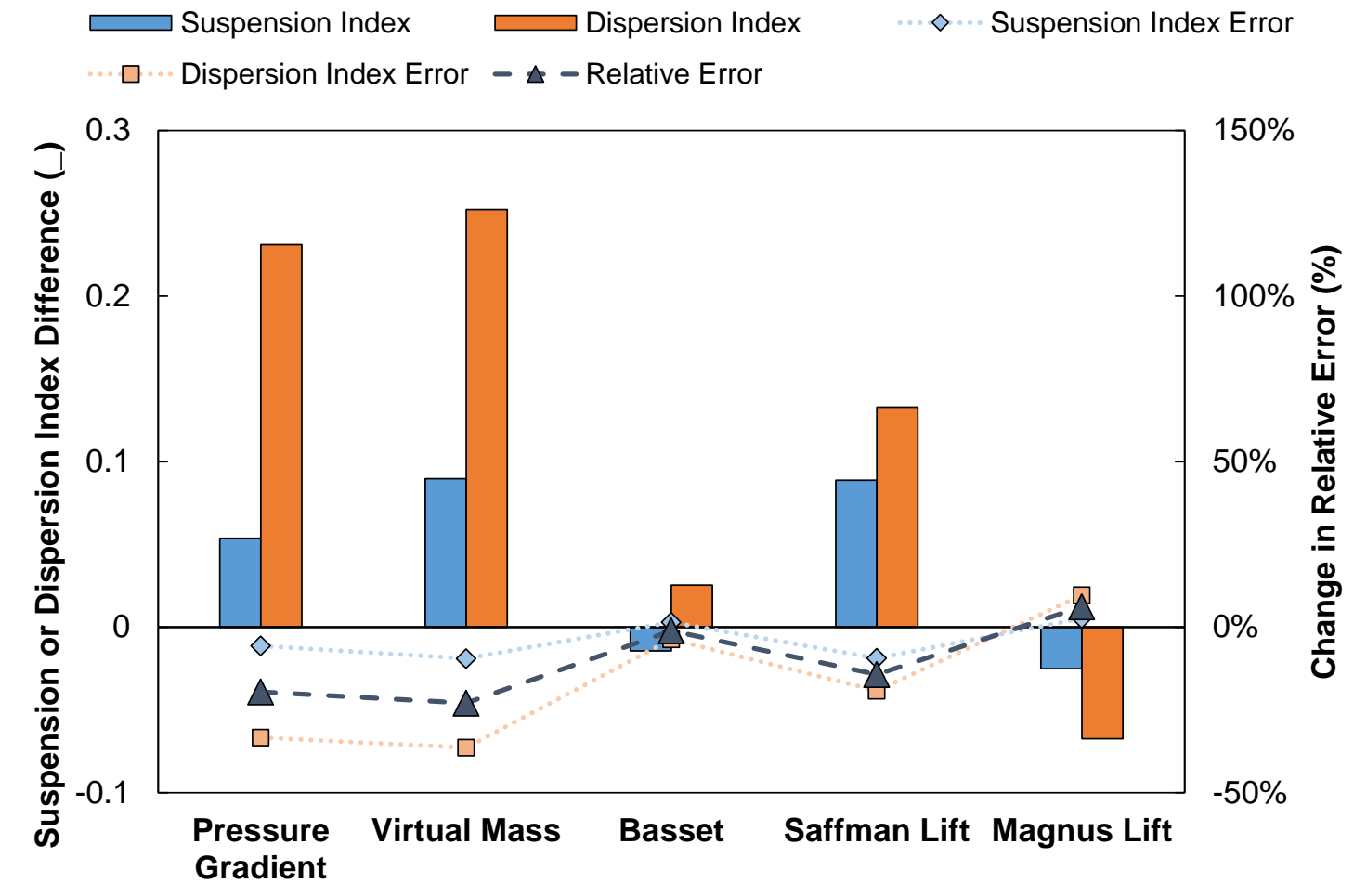

Figure 18. Influence of various fluid-particle interaction forces on suspension and dispersion index differences and change in relative error based on Cases $1 C, 2 A, 2 B$, $2 C, 2 D$, and $2 E$.

The significance of the pressure gradient and virtual mass forces was evaluated by comparing Case $1 \mathrm{C}$ to Cases $2 \mathrm{~A}$ and $2 \mathrm{~B}$. In doing so, it was observed that including these forces increased the mixing indexes and decreased the relative error. The reduction in the relative error confirms that these forces are significant in this application. The pressure gradient force accounts for differences in pressure. These can be caused by (1) the buoyancy of particles and (2) the acceleration of fluid. When taking this force into consideration, the former is overcome by gravitation forces since the particle density is higher than the fluid density. However, the latter is able to promote particle suspension due to the downward pumping flow field. This fluid flow causes the particles to accelerate 
from the bottom of the tank towards the top. Additionally, the differences in pressure caused by the acceleration of the fluid would have also promoted particle dispersion. This is due to the turbulent and locally unsteady nature of the fluid flow field. The dispersion index increased by 0.231 , whereas the suspension index only increased by 0.054 . Therefore, it would appear that the pressure gradient force was a more significant contributor to particle dispersion than it was to suspension. This was also the case for the virtual mass force. This force accounts for the displacement of fluid due to the acceleration of particles. That is to say, it adds the force required to move fluid from the new particle position. Given the moderate viscosity of the liquid, used in this system, this results in a significant increase in particle mobility. More specifically, an increase in particle suspension and dispersion.

Alternatively, when comparing Case $2 \mathrm{E}$ to Case $1 \mathrm{C}$, it was observed that including the Basset force increased dispersion but decreased suspension to a minor extent. This force accounts for the temporal delay in the boundary layer development of accelerating particles caused by viscous effects. Hinsberg et al. [168] took this force into consideration when investigating homogeneous isotropic turbulence flows. In doing so, they found that particle settling is modified in a selective manner based on the particles' Stokes number. Such that, it is enhanced by large Stokes numbers and hindered by small Stokes numbers. Based on the particle size and density used in this study, the Stokes numbers should be relatively large for this application. Therefore, it stands to reason that a minor decrease in particle suspension was observed when implementing the Basset force. Furthermore, Olivieri et al. [160] found that the Basset force also hinders the formation of particle clusters and voided regions. This ultimately increased the dispersion of their 
particles. This was also observed in this study. However, this only resulted in a $1.1 \%$ reduction in the simulation's relative error. Therefore, it was assumed that the Basset force could be neglected with only a minor influence on accuracy and was not included in further simulations to reduce computational costs.

Next, the significance of the Saffman lift force was evaluated by comparing Case $1 \mathrm{C}$ to $2 \mathrm{C}$. In doing so, it was observed that including this force increased both of the mixing indexes and decreased the relative error. This force accounts for the displacement of the particles caused by the shearing of fluid. Due to the rotation of the impeller, large amounts of shear are experienced in this application. Therefore, it is reasonable for the Saffman lift force to be significant.

When comparing Case 2D to Case $1 \mathrm{C}$, it was observed that including the Magnus lift force decreased both the dispersion and suspension indexes. This force accounts for the displacement of particles caused by high particle rotational velocities. These normally occur at the bottom of the tank and around the impeller. Specifically, as a result of the high rates of particle collisions occurring within those regions [92]. These cause the particles to suspend from the bottom of the tank and accumulate at the impeller height [98]. This should result in an increase in particle suspension and a decrease in particle dispersion. However, this was not the case for the current observation. This is most likely due to a limitation associated with the model used to account for the Magnus lift force. In mixing systems, the local fluid vorticity is imparted by impeller motion as well as particle rotational motion. However, with the Magnus lift force model depicted in Equation ( 24 ), this will lead to erroneous results. For example, when the particle rotational velocity is zero and the fluid vorticity is not zero: conceptually, the Magnus lift force should be zero 
and the total lift force should be equal to the Saffman lift force. However, when using Equation ( 24 ), a non-zero Magnus lift force will be calculated instead and the resulting force will even act in the opposite direction of the Saffman lift force. This results in a Magnus lift force that negates the Saffman lift force. In the current work, this unrealistically decreased the degree of particle suspension and increased the relative error by $6.2 \%$. For this reason, the model used for the Magnus force was not taken into consideration in further simulations.

Additionally, Blais et al. [152] also noted that the Magnus lift force may actually be overestimated in general. Since, the interactions between the particle angular velocities and the fluid vorticity field was not accounted for in the numerical model. In this regard, the frictional forces acting on particles by the surrounding fluid has not been taken into consideration. These forces would dampen the particle angular velocities and modify the fluid vorticity field accordingly. As a result, the Magnus lift force would be smaller and less significant. The influence of these friction forces on the particles can be taken into consideration by incorporating a fluid resistance torque into the solid phase torque conservation equation. This was demonstrated by Derksen [98]. Although, this was not investigated further in this work since it is out of scope.

Blais et al. [152] also criticized the fluid-particle interaction force models used to account for the virtual mass, Basset, Saffman lift, and Magnus lift forces. They argued that these equations are inaccurate since they do not account for the presence of neighbouring particles and were derived based on single particle motion. However, the significance of this inaccuracy is still unclear and should be investigated further in future works. 


\subsubsection{Influence of Particle Properties}

In the current study, there is a tendency for particles to accumulate into densely concentrated structures since the particle density is higher than the liquid density. Specifically, the particles tend to accumulate around the tank walls and across the bottom of the tank. For this reason, the motion of the solid phase is not only dependant on fluidparticle interactions, as discussed above. It is also influenced by particle-particle and particle-wall interactions. This can be demonstrated by the following evaluation of various key particle properties used to model particle contacts. In this evaluation, it is reminded that the recommended particle properties, used in most solid-liquid simulations, are based on the measured properties of dry materials. This is an acceptable first estimate for wet particle properties. However, it can lead to significant error and should be corrected by calibrating the particle properties against experimental data. The current work will demonstrate this process by determining the particle properties that minimize the relative error.

The first properties investigated are the sliding and rolling friction coefficients. When increasing these parameters, it was observed that the mixing indexes decreased and the relative error increased. This is illustrated in Figure 19. However, it should be noted that the influence of changing the rolling friction coefficient was more pronounced. 
a)

$\diamond$ Suspension Index $\quad \square-$ Dispersion Index $\quad \cdots \diamond \cdots$ Suspension Index Error

$\square$.... Dispersion Index Error - $\_$- Relative Error

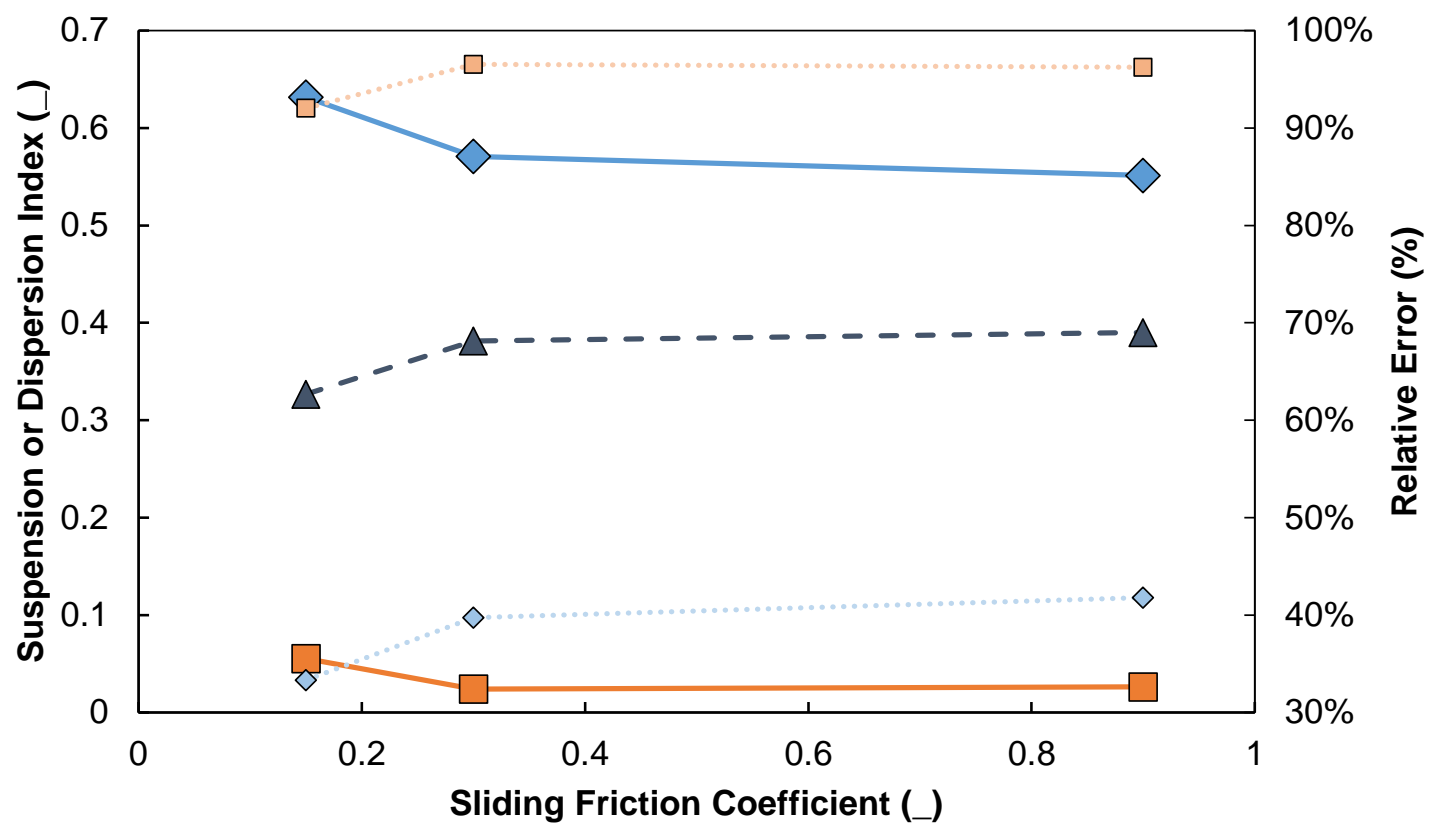

b)

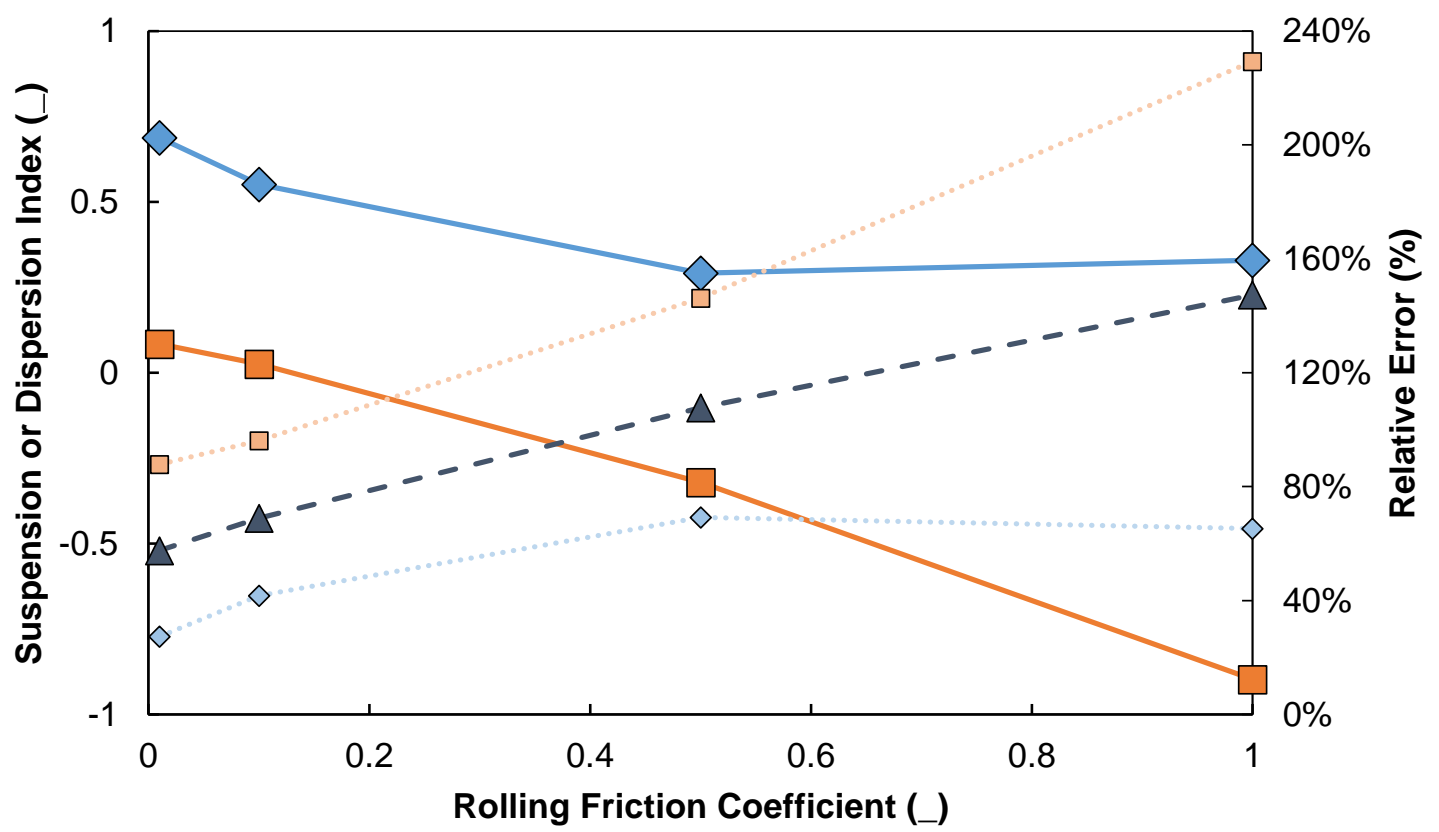

Figure 19. Influence of a) static friction coefficient - based on Cases $1 C, 3 A$, and $3 B-$ and b) rolling friction coefficient - based on Cases $3 B, 3 C, 3 D$, and $3 E$ - on suspension and dispersion indexes. 
The influence of the friction coefficients on the mixing quality is due to the relationship between the friction coefficients and the translational and rotational velocities of the particles. The sliding friction coefficient limits the tangential force that can result from particle-particle and particle-wall collisions, refer to Equation ( 50 ). Increasing this coefficient, increases the limit of the tangential force and the rotational momentum. This consequently increases the particles' rotational velocities. Additionally, increasing the sliding friction coefficient also decreases the translational particle velocities. This was confirmed by comparing the translational and rotational velocities simulated in Cases $1 \mathrm{C}$ and 3B. These cases used respective sliding friction coefficients of 0.3 and 0.9 . In this comparison, the translational and rotational velocities were time-averaged for five seconds after steady-state was reached for all particles. In Case 1C, the average translational and rotational velocities were calculated to be $0.140 \mathrm{~m} / \mathrm{s}$ and $384 \mathrm{RPM}$, respectively. In Case 3B, the averaged translational velocity reduced to a value of 0.129 $\mathrm{m} / \mathrm{s}$, whereas the average rotational velocity increased to a value of 539 RPM. However, this did not significantly influence the particle suspension or dispersion. The rolling friction coefficient, on the other hand, did significantly influence the mixing quality. This parameter directly relates the normal contact force to the rolling resistance torque. Therefore, increasing this coefficient, increases the rolling resistance torque of all particles in contact. This decreases the rotational velocities of all rebounding particles. Additionally, it was observed that increasing the rolling friction coefficient also decreased the translational velocity of particles. This was determined by comparing the average translational and rotational velocities simulated in Cases $3 \mathrm{~B}$ and $3 \mathrm{E}$. These cases used respective rolling friction coefficients of 0.1 and 1 . In Case $3 \mathrm{E}$, the average translational and rotational 
velocities were determined to have significantly decreased to respective values of 0.046 $\mathrm{m} / \mathrm{s}$ and 64 RPM compared to Case 3B. Refer to the sliding friction coefficient discussion above for the values associated with Case 3B. Regardless, for either the sliding or rolling friction coefficients, the translational velocities of the rebounding particles decreased to some degree with increasing coefficients. This resulted in hindered particle mobility leading to a decrease in both particle suspension and dispersion. However, this effect is more pronounced for the rolling friction coefficient since changing this parameter affects all contacting particles. Whereas, changing the sliding friction coefficient only affects a portion of contacting particles that are no longer limited by smaller tangential force limitations. This is demonstrated by the substantial increase in the relative error as the rolling friction coefficient is increased. Although, it should be noted that these results are not consistent with the results of Blais and Bertrand [103]. They found that the fraction of suspended particles increased with increasing friction coefficients. Specifically, at intermediate speeds. However, their simulations were performed for a different flow regime with a more viscous liquid phase.

The simulation results presented in this work also suggest that wet particles have lower sliding and rolling friction coefficients. This stands to reason since liquid would act as a lubricant between the particles and reduce the friction experienced during collisions. However, in the current study, reducing the sliding friction coefficient also resulted in instabilities within the simulations. Alternatively, increasing this coefficient resulted in insignificant changes to the predicted mixing quality and shorter computational runtimes (i.e. the amount of time required to reach steady-state decreased). For these reasons, the sliding friction coefficient was artificially increased in further simulations in an attempt 
to reduce computational costs and promote stability. These instabilities were not observed with the rolling friction coefficient. Therefore, it is recommended that only reduced rolling friction coefficients be used to improve the accuracy of the simulations. In the present work, the sliding friction coefficient was increased from 0.3 to 0.9 . While, the rolling friction coefficient was decreased from 0.1 to 0.01 . This is depicted in Cases $1 \mathrm{C}$ and $3 \mathrm{C}$. As a result, the relative error and the computational runtime were reduced by respective percentages of $10.5 \%$ and $26.9 \%$.

The second particle property investigated is the Young's Modulus. When increasing this parameter, it was observed that the mixing indexes and relative errors mildly varied. This is illustrated in Figure 20. These trends do not appear to be significant as the relative error only varies by a maximum of $4.2 \%$. This is consistent with the results of Blais and Bertrand [103]. They also found that modifying the Young's modulus had little to no effect on the fraction of suspended particles.

This finding confirms that stiffness scaling can be used in CFD-DEM simulations with only a minor impact on accuracy. In doing so, the Young's modulus can artificially be decreased in order to increase the DEM time step. This results in a reduction in the computational runtime [169]. In the present study, decreasing the Young's Modulus from 100 MPA to $10 \mathrm{MPa}$ reduced the computational runtime by $60.4 \%$. This was determined by comparing the computational runtimes of Cases $3 \mathrm{G}$ and $3 \mathrm{~B}$. This method has also been used in other studies investigating solid-liquid system with CFD-DEM coupling. Han et al. [116], who studied a circulating fluidized bed, reduced their Young's modulus by an order of magnitude. In doing so, they found that the Young's modulus only had a minor 
impact on the particles' mean residence time. In addition to this, Di Renzo and Di Maio [119] and Di Renzo et al. [118] also applied stiffness scaling in fluidized bed applications.

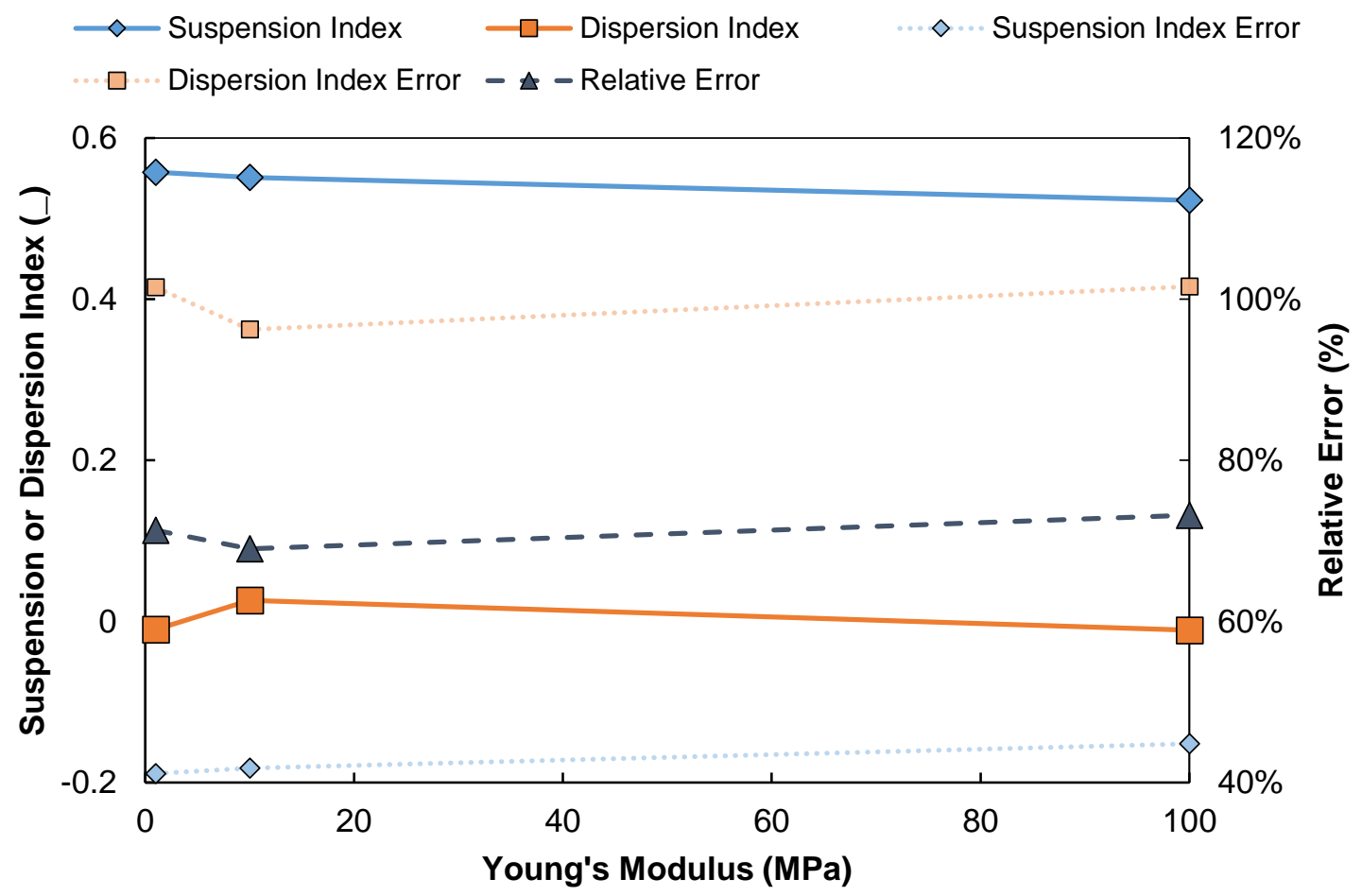

Figure 20. Influence of Young's Modulus on suspension and dispersion indexes based on Cases 3B, 3F, and 3G.

The last particle property investigated is the coefficient of restitution. When decreasing this parameter, it was observed that the mixing indexes decrease and the relative error increases. This is illustrated in Figure 21. Varying the coefficient of restitution was used as an approximation for the lubrication force. This force hinders contacting particles from moving towards and away from each other. Based on the empirical correlation proposed by Joseph et al. [136], the lubrication force becomes more pronounced as the coefficient of restitution decreases. Therefore, by decreasing 
coefficient of restitution, it is expected that both mixing indexes would decrease. This was confirmed with the simulation results. It should be noted that this finding was not consistent with the results of Blais and Bertrand [103]. Rather, they found that the fraction of suspended particles was not affected by varying the coefficient of restitution. However, this discrepancy is realistic since viscous forces were dominant compared to the lubrication force in their study. Therefore, modifying the coefficient of restitution and artificially accounting for the lubrication force resulted in little to no effect.

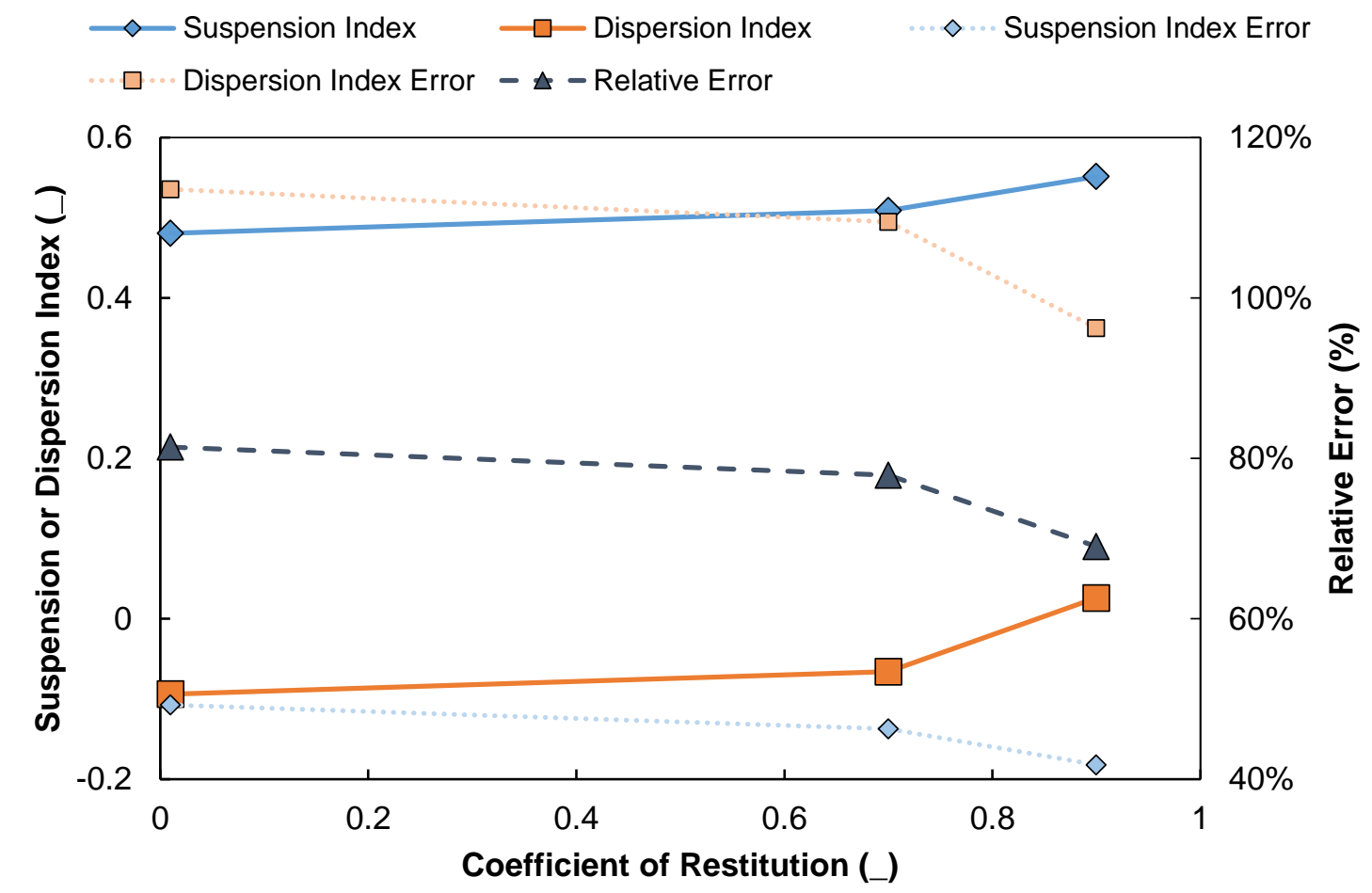

Figure 21. Influence of coefficient of restitution on suspension and dispersion indexes based on Cases 3B, 3H, and 31 .

It is reminded that changing the global coefficient of restitution was only used as a crude approximation for the lubrication force. However, the results can still be used to 
evaluate the force's significance, which would most likely be significant in this system. This can be confirmed based on the significant change in the mixing quality resulting from minor changes in the global coefficient of restitution (i.e. by comparing Cases 3B and 3I). However, it is recommended that this be confirmed in future works by accounting for the lubrication force using a more appropriate method. More specifically, by dynamically changing the coefficient of restitution for each individual particle based on it instantaneous Stokes number. 


\section{Chapter 6 : Conclusions and Recommendations}

In this study, a solid-liquid mixing system in the turbulent regime was studied comprehensively via numerical and experimental methods. The spatial distribution of the particle concentrations was measured using ERT. These measurements were summarized into mixing indexes, which quantified both the degree of particle suspension and dispersion. Current mixing index definitions only focus on the degree of particle dispersion throughout the tank. Therefore, a new suspension mixing index was proposed in this study and applied based on the ERT measurement capabilities. Moreover, a twoway coupled CFD-DEM model was developed using ANSYS Fluent v16.2 and EDEM v2.7/v2017 software packages.

Using the mixing indexes, the simulation and experimental results were compared. In doing so, it was found that the model underestimated both the degree of suspension and dispersion. This was most likely due to model limitations. Specifically, those associated with the mesh size, the MRF method being applied to a Eulerian-Lagrangian 
simulation, and the fluid-particle interaction force models as well as the turbulence closure equations. The sensitivity of the model to changes in various model parameters was also investigated. In doing so, it was found that the O'Brien drag model was better suited for this application compared to the Di Felice and Gidaspow models, which underestimated either the degree of suspension or dispersion due to their respective model restrictions. Additionally, it was determined that the pressure gradient, virtual mass, and Saffman lift forces needed to be taken into consideration in this application. By including these forces, both the degree of suspension and dispersion were improved. Alternatively, the influence of the Basset force on particle suspension and dispersion was found to be negligible. While, including the Magnus lift force was found to result in erroneous behaviour. This can be attributed to the form of the model used to account for it. Lastly, it was also found that the degree of suspension and dispersion significantly increases with decreasing rolling friction coefficients as well as increasing coefficients of restitution. While, the sliding friction coefficient and Young's modulus did not significantly influence the mixing quality. The latter confirmed that stiffness scaling can be used in this study. Using this method, the computational runtime was reduced considerably without compromising the simulations' accuracy.

In future works, it is recommended that smaller particles be used in the developed model. Using smaller particles would allow smaller mesh sizes to be used, which would result in more accurate fluid flow predictions and consequently more accurate fluidparticle interaction force predictions. Decreasing the particle diameters and mesh sizes, increases the computational requirements. However, based on the results of the current study, stiffness scaling can then be used to combat the increase in computational costs. 
Moreover, it is recommended that the lubrication force be taken into consideration through a dynamic coefficient of restitution - to model this force more realistically - in future simulations. Additional attempts to improve the simulation accuracy can include replacing MRF with alternative approaches such as a sliding mesh; or evaluating the influence of more sophisticated drag and turbulence models (e.g. Reynolds Stress model) on the simulation results. 


\section{APPENDIX A. Di Felice User Defined Function}

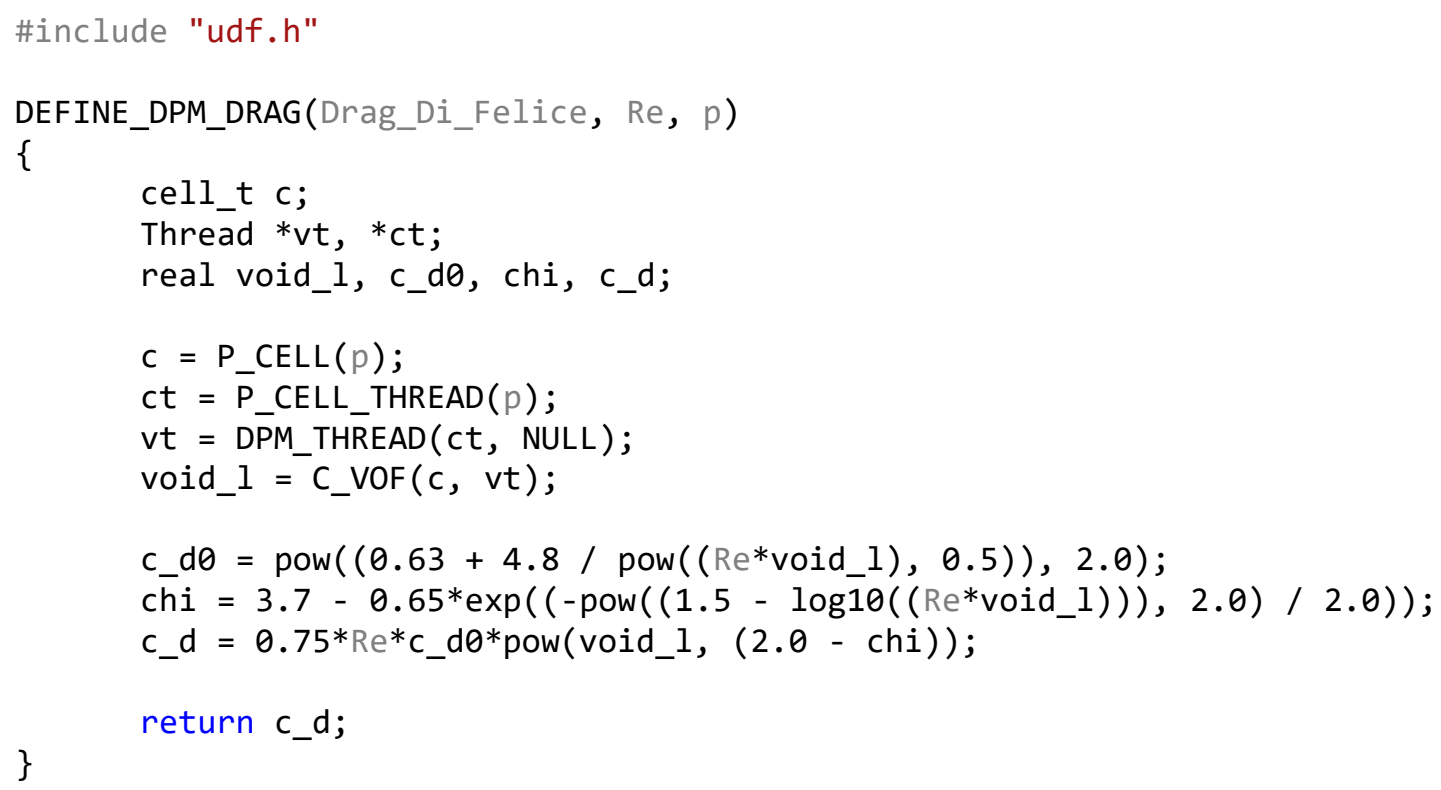




\section{APPENDIX B. Magnus Lift Force User Defined Function}

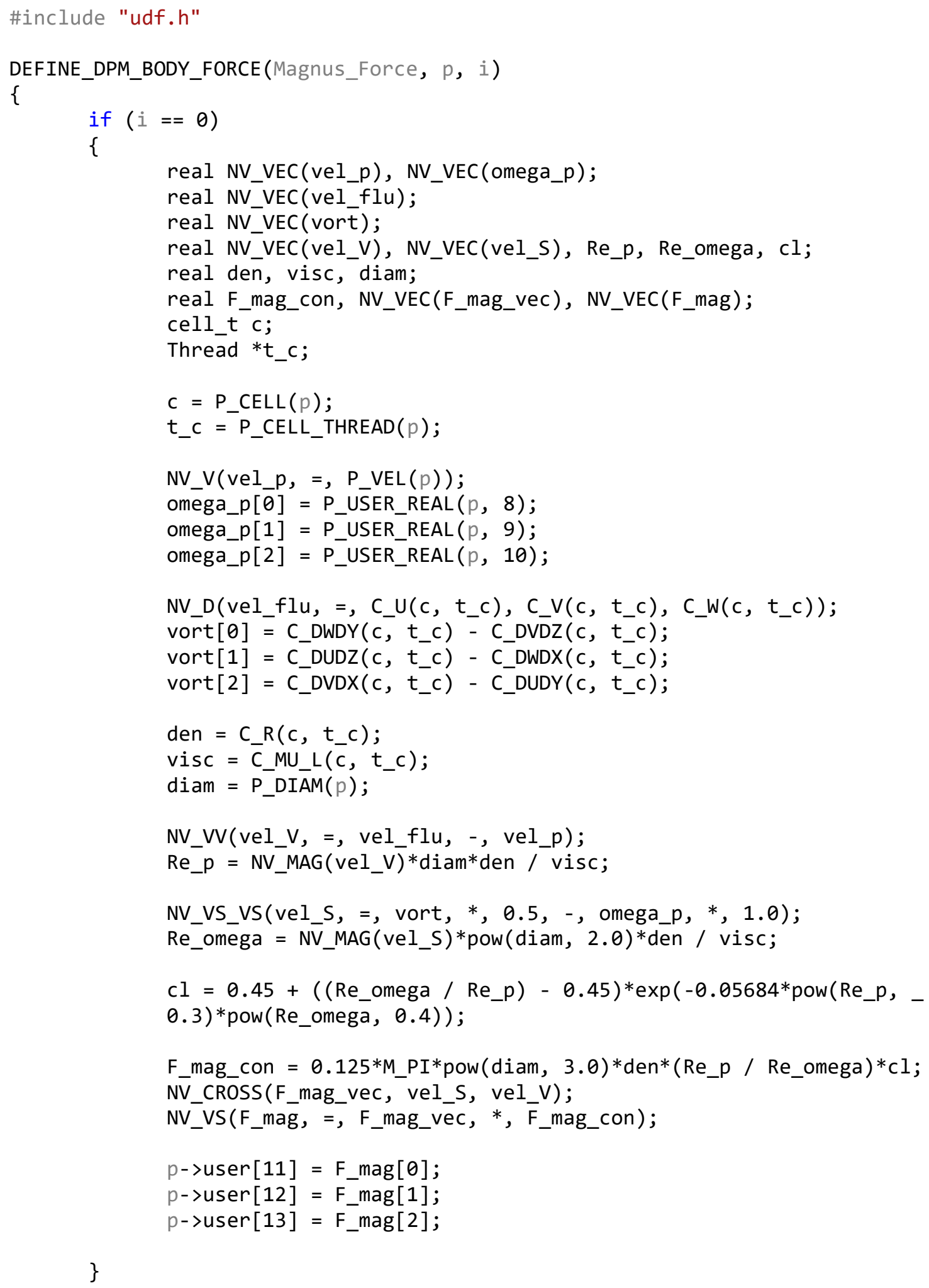


\} return (P_USER_REAL $(p,(i+11)) /$ P_MASS $(p))$;

Note: This UDF required that the number of User Variables in the coupling interface be increased to 14. Additionally, modifications in the coupling interface were required to allow EDEM to communicate the particle angular velocities to Fluent. Refer to APPENDIX C. 


\section{APPENDIX C. Modifications to Coupling Interface (Supplement to APPENDIX B)}

I. Modifications to "AdaptorInterface.cpp" file (found in coupling interface installation folder at “...ledem_coupling_buildledem_cfd_adaptor_build|src”):

a. Replaced lines 284-286 with the following lines:

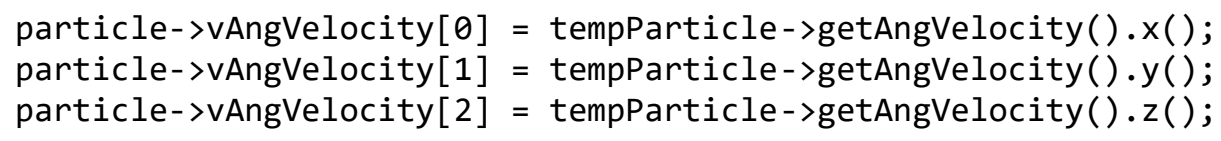

II. Modifications to "edem_coupling. $h$ "' file (found in coupling interface installation folder at “...ledem_coupling_buildllib_edem_coupling|src"):

a. Inserted following lines at line 49:

DEM_OMEGA_X, DEM_OMEGA_Y, DEM_OMEGA_Z, ADDED_FP_X, ADDED_FP_Y, ADDED_FP_Z,

b. Inserted following lines to new line 65 :

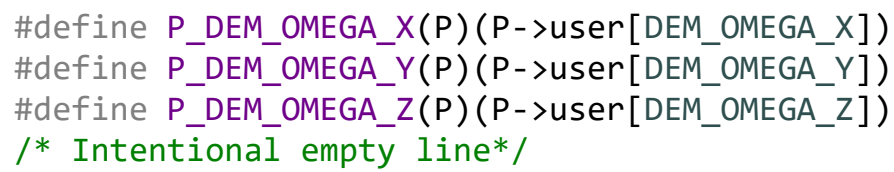

III. Modifications to "edem_coupling.c" file (found in coupling interface installation folder at “...ledem_coupling_buildllib_edem_coupling $\mid s r c ")$ :

a. Inserted following lines to line 314 :

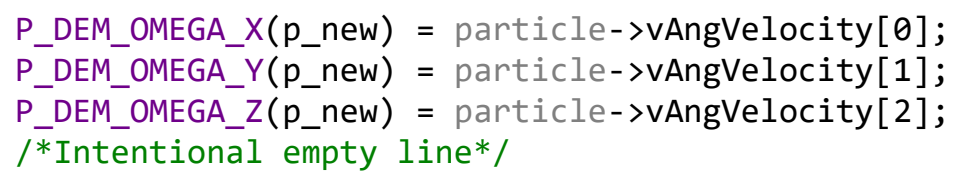




\section{APPENDIX D. Basset Force User Defined Function}

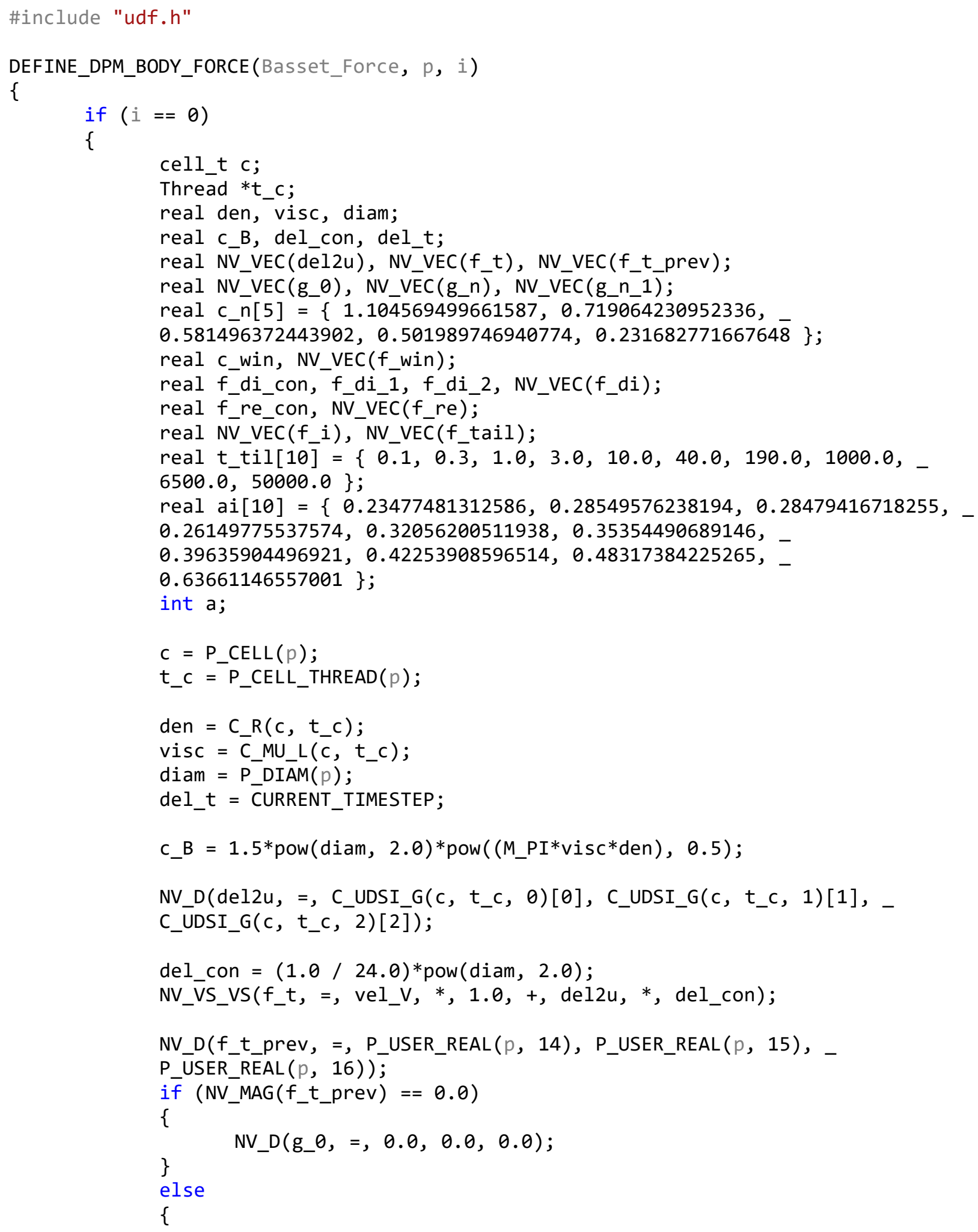




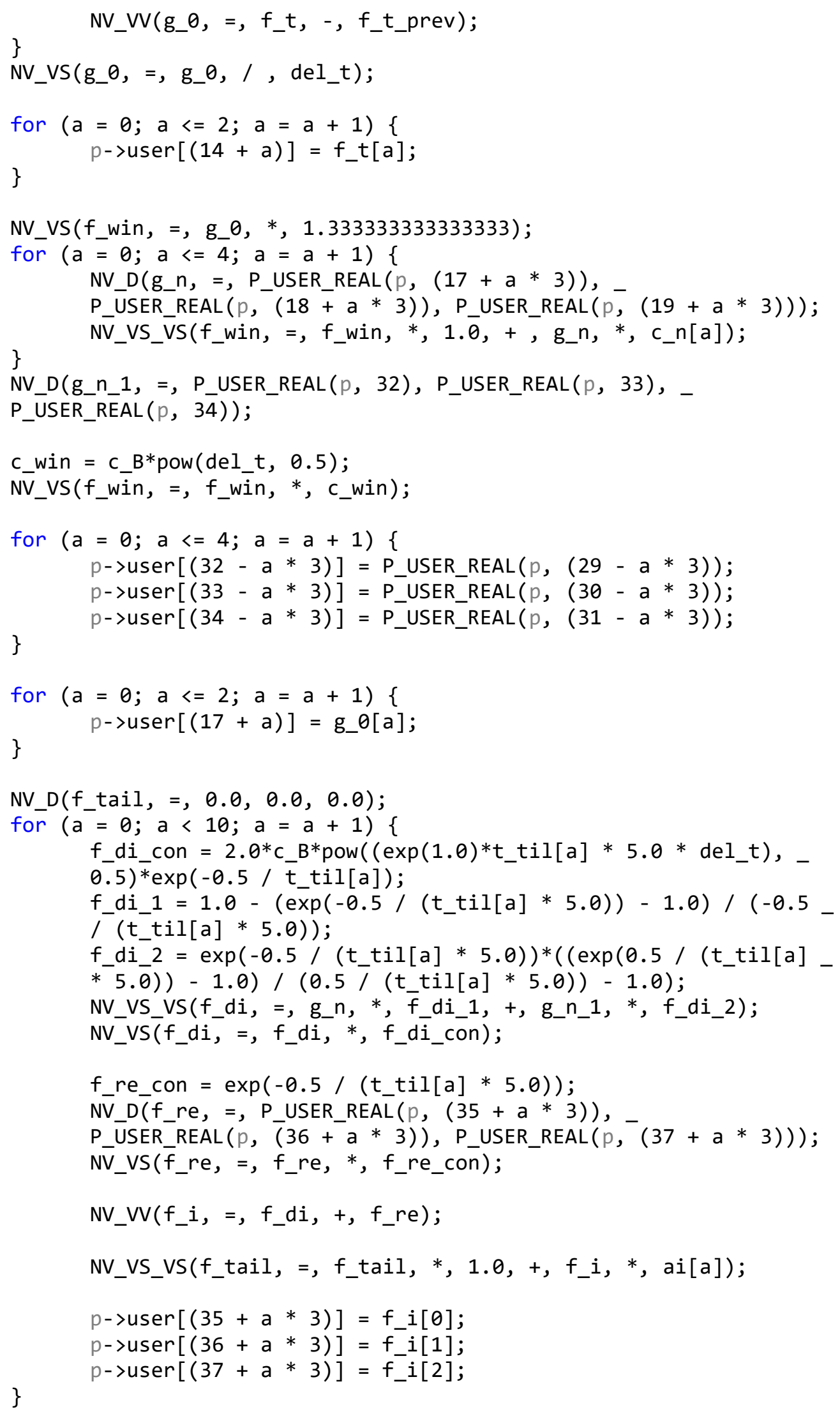




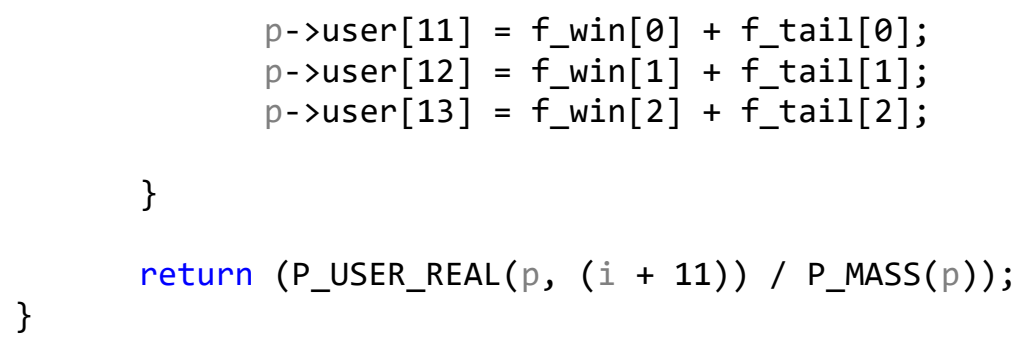

Note: This UDF required that the number of User Variables in the coupling interface be increased to 65. Additionally, the adjust UDF depicted in APPENDIX E was required to determine the Laplacian of the fluid velocity. Furthermore, modifications in the coupling interface were required to prevent the data stored in the User Variables from being deleted during the coupling process. Refer to APPENDIX F.

Additional Note: Two body force UDFs cannot be used at one time. Therefore, when implementing both the Magnus and Basset forces, the UDF depicted in APPENDIX B was combined with this UDF. 


\section{APPENDIX E. Adjust User Defined Function (Supplement to APPENDIX D)}

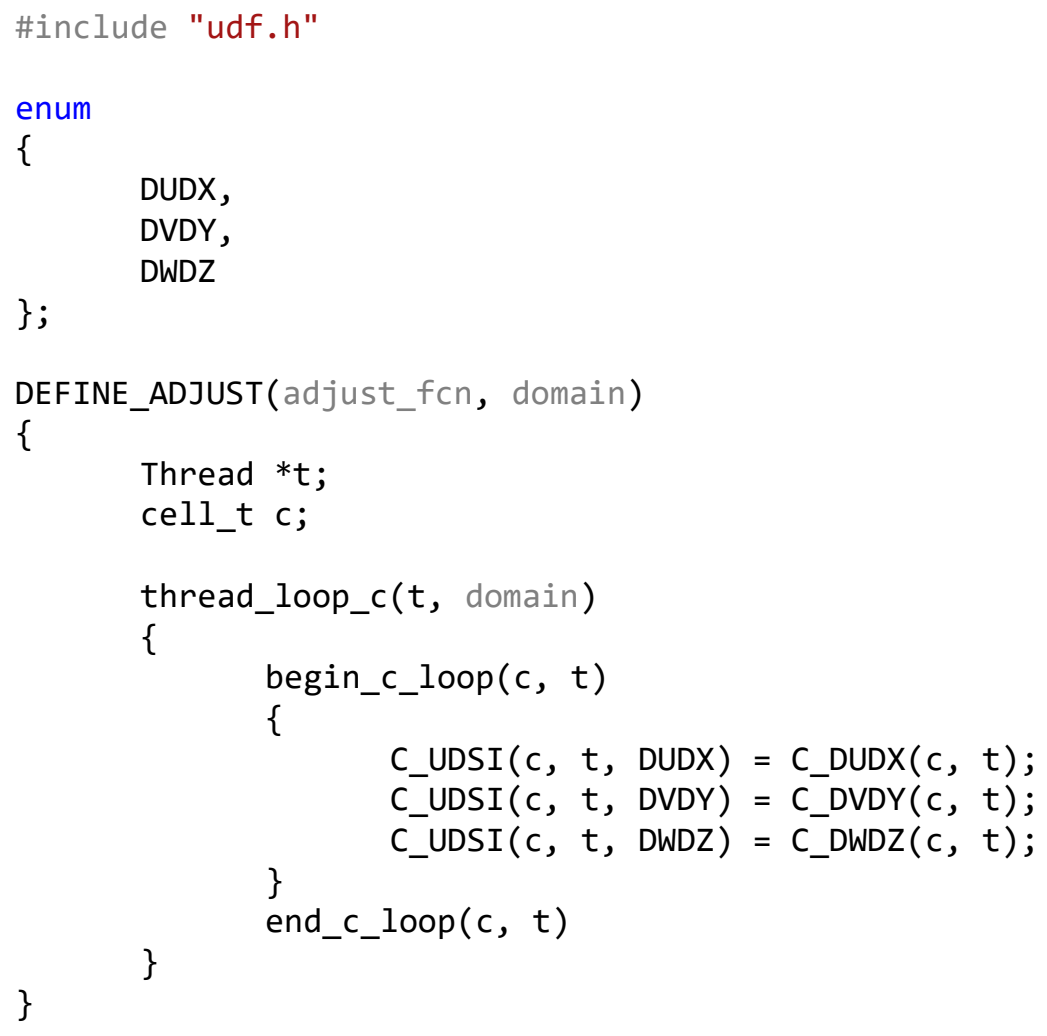

Note: This UDF required that 3 User Defined Scalars be created. 


\section{APPENDIX F. Modifications to Coupling Interface (Supplement to APPENDIX D)}

I. Modifications to "edem_coupling.h"'" file (found in coupling interface installation folder at “...ledem_coupling_buildllib_edem_coupling $\mid s r c "):$

a. Inserted following lines at line 33:

int ftprev_property_index;

int gn_property_index;

int fi_property_index;

cxboolean basset_registered;

cxboolean basset_updated;

b. Inserted following lines at new line 54 :

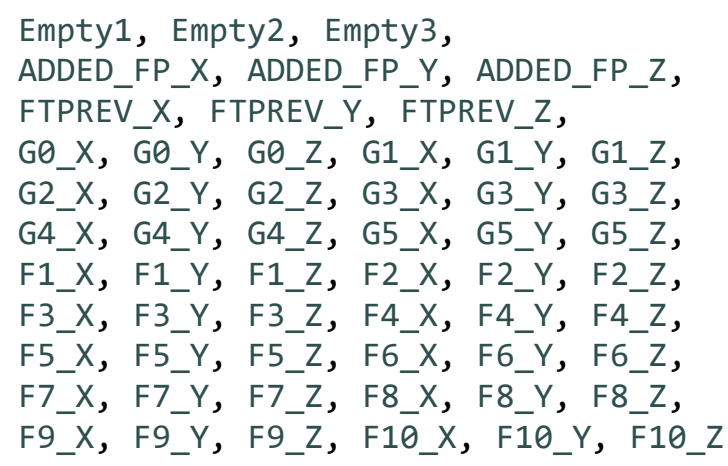

c. Inserted following lines to new line 79:

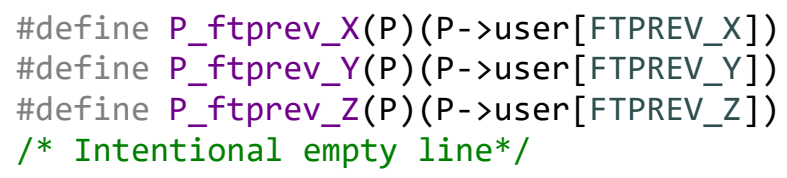

II. Modifications to "edem_coupling.c" file (found in coupling interface installation folder at “...ledem_coupling_buildllib_edem_coupling|src"):

a. Inserted following lines at line 53:

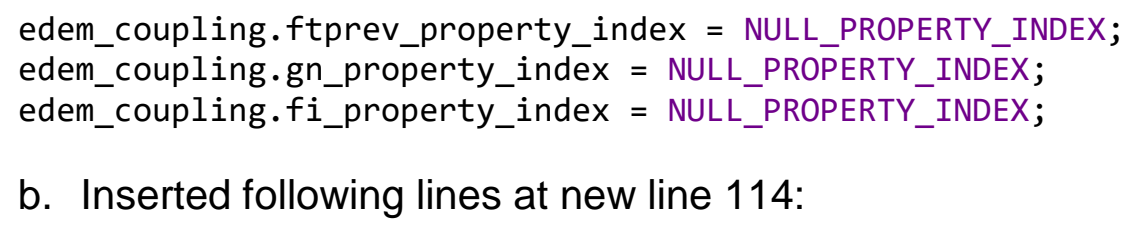

b. Inserted following lines at new line 114: 


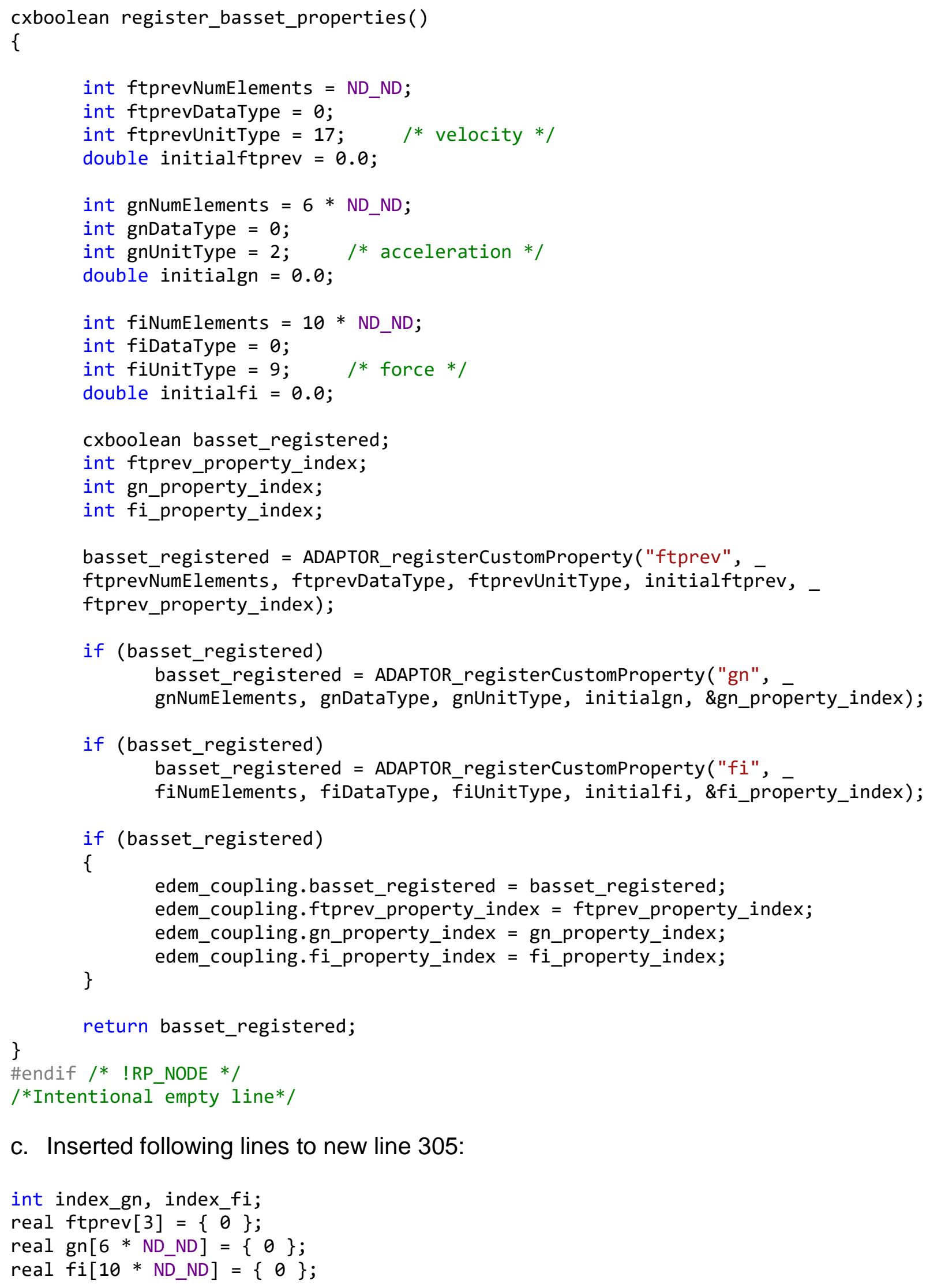

c. Inserted following lines to new line 305:

int index_gn, index_fi;

real ftprev[3] $=\{\bar{\theta}\}$;

real gn $[6 *$ ND_ND $]=\{\theta\}$;

real fi $[10 *$ ND_ND $]=\{\theta\}$; 
d. Inserted following lines to new line 349:

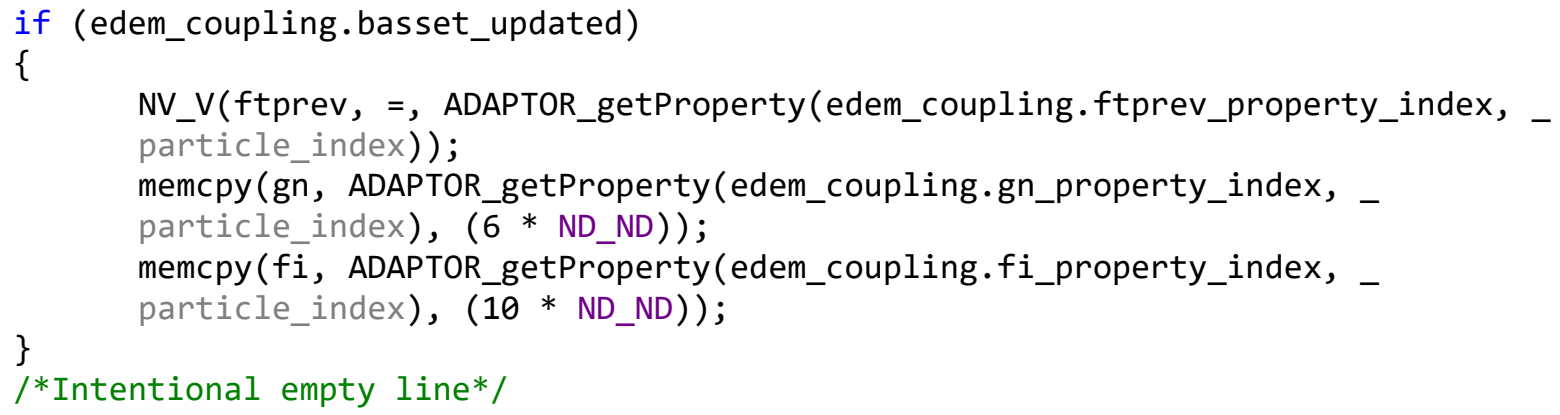

e. Inserted following lines to new line 371:

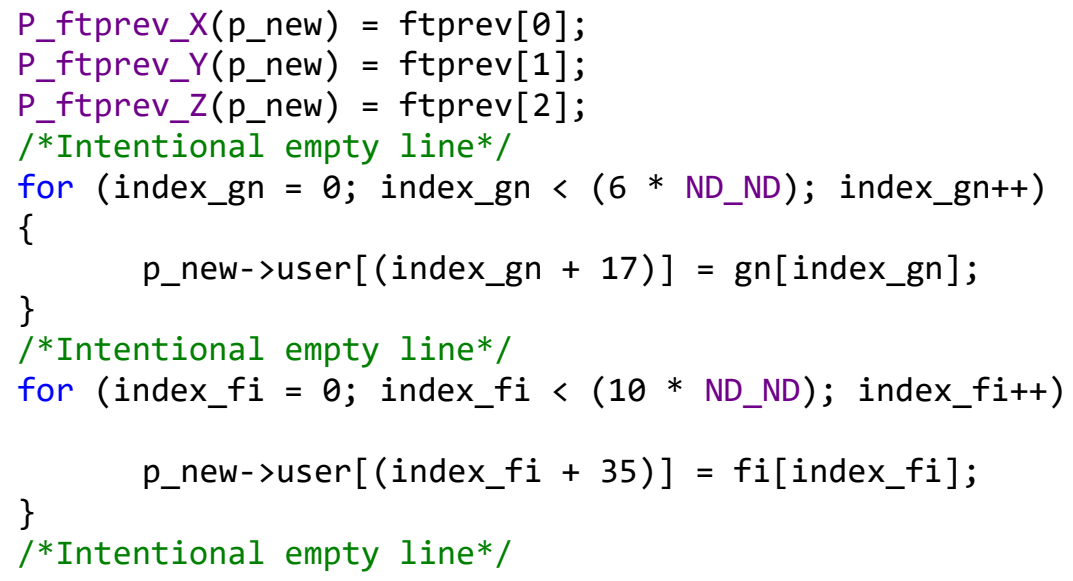

f. Inserted following lines to new line 473:

if (!edem_coupling.basset_registered)

g. Inserted following lines to new line 482:

ADAPTOR_updateValuesForProperty(num_particles, edem_coupling.ftprev_property_index); ADAPTOR_updateValuesForProperty(num_particles, edem_coupling.gn_property_index); ADAPTOR_updateValuesForProperty(num_particles, edem_coupling.fi_property_index); /*Intentional empty line*/

h. Inserted following lines to new line 531:

void update_basset_properties()

\{

double *ftprev, *gn, *fi;

double *offset_ftprev, *offset_gn, *offset_fi;

int index_gn, index_fi; 


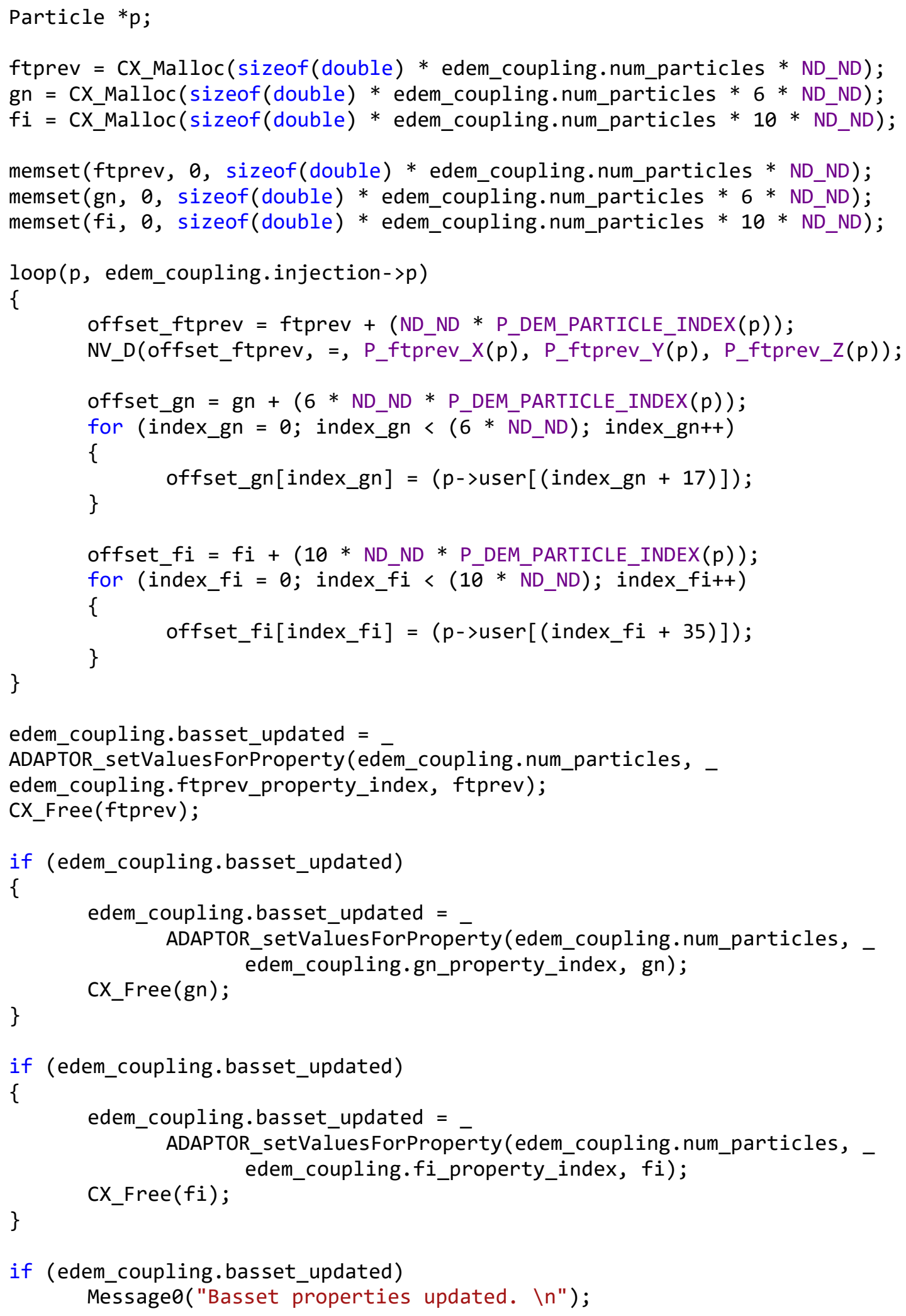


i. Inserted following lines at new line 977:

update_basset_properties( ) ;

/*Intentional empty line*/

III. Modifications to "compute_particle_forces.c" file (found in coupling interface installation folder at “...ledem_coupling_buildllib_edem_coupling|src"):

a. Inserted following line at line 35 :

int index_gn, index_fi;

b. Inserted following lines at new line 87 :

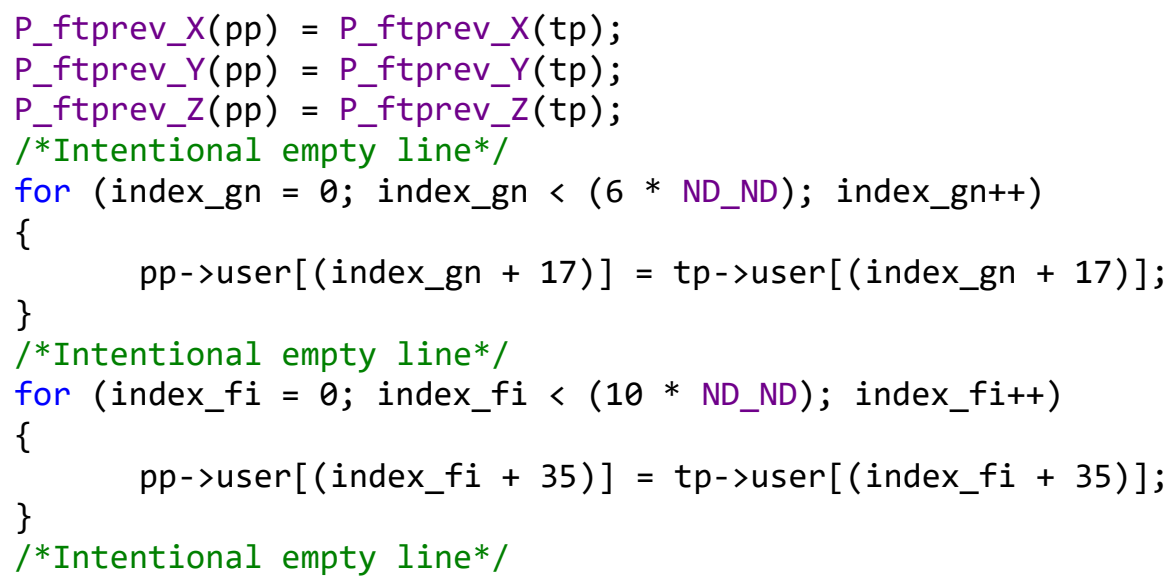




$N$
$D$
$C$
$T$
$H$
$V$
$\underline{v}$
$I$
$\underline{C}$
$\sigma$
$\underline{Y}$
$S$
$n$
$M$
$N$
$X$
$X_{p}$
$\overline{X_{p}}$
$X_{r, p}$
$X_{r, p}$
$X_{k, p}$
$\overline{X_{k, p}}$
$X_{p, a v g}$
$X_{r, a v g}$
$X_{k, a v g}$
$X_{t}$
$\boldsymbol{u}$

Impeller speed

$s^{-1}$

Impeller diameter $m$

Impeller clearance $m$

Tank diameter $m$

Liquid level height $m$

Voltage value $\quad \mathrm{V}$

Voltage readings vector $\quad V$

Injection current $A$

Injection current vector $\quad A$

Conductivity value $\quad S \cdot m^{-1}$

Conductivity matrix $S \cdot \mathrm{m}^{-1}$

Sensitivity map value

Nodal point location

Number of voltage measurements

Number of tomograph elements

Particle volume fraction

Average concentration in plane $p$

Total average of the $X_{p}$ values

Average concentration in ring $r$ and plane $p$

Total average of the $X_{r, p}$ values

Concentration in element $\mathrm{k}$ and plane $\mathrm{p}$

Total average of the $X_{k, p}$ values

Average of the $X_{r, p}$ or $X_{k, p}$ values in the same plane $\mathrm{p}$

Average of $X_{r, p}$ values in the same annular section $r$

Average of the $X_{k, p}$ values in the same element section

True system concentration throughout the tank

Fluid velocity vector

$m \cdot s^{-1}$ 


\begin{tabular}{|c|c|c|}
\hline$\omega_{c}$ & Fluid vorticity & $s^{-1}$ \\
\hline$\varepsilon_{f}$ & Fluid porosity & - \\
\hline$\rho_{f}$ & Fluid density & $\mathrm{kg} \cdot \mathrm{m}^{-3}$ \\
\hline$\mu_{f}$ & Fluid dynamic viscosity & $P a \cdot s$ \\
\hline$\nabla p$ & Fluid pressure gradient & $P a$ \\
\hline $\boldsymbol{\tau}$ & Fluid viscous stress tensor & $P a$ \\
\hline$g$ & Acceleration due to gravity & $m \cdot s^{-1}$ \\
\hline$F_{p f}$ & Volume-averaged fluid-particle interaction force & $N$ \\
\hline$\Delta V$ & Volume of the fluid mesh cell & $m^{3}$ \\
\hline$u_{r}$ & Moving reference frame relative fluid velocity & $m \cdot s^{-1}$ \\
\hline$\omega_{M R F}$ & Moving reference frame angular velocity & $s^{-1}$ \\
\hline$r_{M R F}$ & Moving reference frame position vector & $m$ \\
\hline$v$ & Particle translational velocity & $m \cdot s^{-1}$ \\
\hline $\mathbf{v}_{r}$ & Particle relative velocity & $m \cdot s^{-1}$ \\
\hline$\omega$ & Particle angular velocity & $s^{-1}$ \\
\hline$d_{p}$ & Particle diameter & $m$ \\
\hline$R$ & Particle radius & $m$ \\
\hline$V_{p}$ & Particle volume & $m^{3}$ \\
\hline$\rho_{\mathrm{p}}$ & Particle density & $\mathrm{kg} \cdot \mathrm{m}^{-3}$ \\
\hline $\mathrm{w}_{\mathrm{p}}$ & Particle weight fraction & - \\
\hline$m$ & Particle mass & $\mathrm{kg}$ \\
\hline$I_{p}$ & Particle moment of inertia & $\mathrm{kg} \cdot \mathrm{m}^{2}$ \\
\hline$k$ & Particle elastic spring stiffness & $N \cdot m^{-1}$ \\
\hline$\delta$ & Particle overlap (displacement) & $m$ \\
\hline$\eta$ & Particle dissipative viscous damping & $N \cdot s \cdot m^{-1}$ \\
\hline$\mu_{s}$ & Particle sliding friction coefficient & - \\
\hline$\mu_{r}$ & Particle rolling friction coefficient & - \\
\hline Y & Particle Young's modulus & $P a$ \\
\hline$G$ & Particle Shear modulus & $P a$ \\
\hline$v$ & Particle Poisson ratio & - \\
\hline
\end{tabular}




\begin{tabular}{|c|c|c|}
\hline$e_{r}$ & Particle coefficient of restitution & - \\
\hline$R_{c}$ & Distance from the particle center to the contact plane & $m$ \\
\hline$f^{c}$ & Particle contact force & $N$ \\
\hline$f_{p f}$ & Individual fluid-particle interaction force & $N$ \\
\hline$f_{d}$ & Drag force & $N$ \\
\hline$f_{\nabla p}$ & Pressure gradient force & $N$ \\
\hline$f_{v m}$ & Virtual mass force & $N$ \\
\hline$f_{\text {Bass }}$ & Basset force & $N$ \\
\hline$f_{\text {Saff }}$ & Saffman lift force & $N$ \\
\hline$f_{M a g}$ & Magnus lift force & $N$ \\
\hline$f_{\text {lift }}$ & Total lift force & $N$ \\
\hline$f^{\prime \prime}$ & Additional fluid-particle interaction forces & $N$ \\
\hline$M_{r}$ & Particle rolling torque & $N \cdot m$ \\
\hline$M_{t}$ & Particle tangential torque & $N \cdot m$ \\
\hline$\beta_{p f}$ & Drag coefficient & $N \cdot s \cdot m^{-1}$ \\
\hline$v_{r s}$ & Ratio of terminal velocities - single particle to cluster of particles & - \\
\hline$R e, R e_{p}$ & Particle Reynolds number & - \\
\hline$R e_{\omega}$ & Particle rotational Reynolds number & - \\
\hline$R e_{N}$ & Modified Reynolds number for mixing tanks & _ \\
\hline$\tau_{\mathrm{DEM}}$ & DEM time step & $s$ \\
\hline$\tau_{\mathrm{CFD}}$ & CFD time step & $s$ \\
\hline$S I$ & Suspension index & - \\
\hline$D I$ & Dispersion index & - \\
\hline
\end{tabular}




\section{Subscripts}

liq Conductive liquid phase

nc Non-conductive dispersed phase

$m c \quad$ Measured conductivity phase

cu Nodal point for current injection

vo Nodal point of voltage detection

ref Reference reading

$P \quad$ Plane $\mathrm{p}$

$R \quad$ Ring $r$

$K \quad$ Element k

$x \quad \mathrm{X}$ direction

$y \quad \mathrm{Y}$ direction

$z \quad$ Axial or $z$ direction

$r$ Radial direction

$t$ Overall

$f \quad$ Fluid phase

$p \quad$ Particle phase

$i, j \quad$ Particle index

eq Equivalent term

$n \quad$ Normal direction

$t \quad$ Tangential direction

exp Experimental terms

sim Simulation terms 


\section{References}

[1] R. E. Shah, R. S. Shah, B. Sajjadi, A. Raman, A. Aziz, and S. Ibrahim, "Solid-liquid mixing analysis in stirred vessels," Rev. Chem. Eng., vol. 31, no. 2, pp. 119-147, 2015.

[2] L. Pakzad, F. Ein-Mozaffari, and P. Chan, "Measuring Mixing Time in the Agitation of Non-Newtonian Fluids through Electrical Resistance Tomography," Chem. Eng. Technol., vol. 31, no. 12, pp. 1838-1845, 2008.

[3] M. Sharifi and B. Young, "Electrical resistance tomography (ERT) applications to chemical engineering," Chem. Eng. Res. Des., vol. 91, no. 9, pp. 1625-1645, 2013.

[4] S. T. Harrison, R. Stevenson, and J. J. Cilliers, "Assessing solids concentration homogeneity in Rushton-agitated slurry reactors using electrical resistance tomography (ERT)," Chem. Eng. Sci., vol. 71, pp. 392-399, 2012.

[5] J. Aubin, D. F. Fletcher, and C. Xuereb, "Modeling turbulent flow in stirred tanks with CFD: the influence of the modeling approach, turbulence model and numerical scheme," Exp. Therm. Fluid Sci., vol. 28, no. 5, pp. 431-445, 2004.

[6] D. Guha, P. A. Ramachandran, M. P. Dudukovic, and J. J. Derksen, "Evaluation of large Eddy simulation and Euler-Euler CFD models for solids flow dynamics in a stirred tank reactor," AIChE J., vol. 54, no. 3, pp. 766-778, 2008.

[7] G. R. Kasat, A. R. Khopkar, V. V. Ranade, and A. B. Pandit, "CFD simulation of liquid-phase mixing in solid-liquid stirred reactor," Chem. Eng. Sci., vol. 63, no. 15, pp. 3877-3885, 2008. 
[8] G. Micale, F. Grisafi, L. Rizzuti, and A. Brucato, "CFD simulation of particle suspension height in stirred vessels," Chem. Eng. Res. Des., vol. 82, no. 9, pp. 1204-1213, 2004.

[9] G. Micale, G. Montante, F. Grisafi, A. Brucato, and J. Godfrey, "CFD simulation of particle distribution in stirred vessels," Chem. Eng. Res. Des., vol. 78, no. 3, pp. 435$444,2000$.

[10] G. Montante, G. Micale, F. Magelli, and A. Brucato, "Experiments and CFD predictions of solid particle distribution in a vessel agitated with four pitched blade turbines," Chem. Eng. Res. Des., vol. 79, no. 8, pp. 1005-1010, 2001.

[11] B. N. Murthy, R. S. Ghadge, and J. B. Joshi, "CFD simulations of gas-liquid-solid stirred reactor: Prediction of critical impeller speed for solid suspension," Chem. Eng. Sci., vol. 62, no. 24, pp. 7184-7195, 2007.

[12] S. Hosseini, D. Patel, F. Ein-Mozaffari, and M. Mehrvar, "Study of solid-liquid mixing in agitated tanks through computational fluid dynamics modeling," Ind. Eng. Chem. Res., vol. 49, no. 9, pp. 4426-4435, 2010.

[13] M. A. Van der Hoef, M. Ye, M. van Sint Annaland, A. T. Andrews, S. Sundaresan, and J. A. M. Kuipers, "Multiscale modeling of gas-fluidized beds," Adv. Chem. Eng., vol. 31, pp. 65-149, 2006.

[14] H. P. Zhu, Z. Y. Zhou, R. Y. Yang, and A. B. Yu, "Discrete particle simulation of particulate systems: theoretical developments," Chem. Eng. Sci., vol. 62, no. 13, pp. 3378-3396, 2007. 
[15] Z. Li, J. J. Derksen, and Z. Gao, "Models and Applications for Simulating Turbulent Solid-Liquid Suspensions in Stirred Tanks," J. Chem. Eng. Jpn., vol. 48, no. 5, pp. 329-336, 2015.

[16] M. A. Van der Hoef, M. van Sint Annaland, N. G. Deen, and J. A. M. Kuipers, "Numerical simulation of dense gas-solid fluidized beds: A multiscale modeling strategy," Annu Rev Fluid Mech, vol. 40, pp. 47-70, 2008.

[17] V. A. Atiemo-Obeng, W. R. Penney, and P. Armenante, Solid-liquid mixing. Wiley: Hoboken, NJ, 2004.

[18] R. Jafari, J. Chaouki, and P. A. Tanguy, "A comprehensive review of just suspended speed in liquid-solid and gas-liquid-solid stirred tank reactors," Int. J. Chem. React. Eng., vol. 10, no. 1, 2012.

[19] E. L. Paul, V. A. Atiemo-Obeng, and S. M. Kresta, Handbook of industrial mixing: science and practice. John Wiley \& Sons, 2004.

[20] S. Hosseini, D. Patel, F. Ein-Mozaffari, and M. Mehrvar, "Study of solid-liquid mixing in agitated tanks through electrical resistance tomography," Chem. Eng. Sci., vol. 65, no. 4, pp. 1374-1384, 2010.

[21] T. N. Zwietering, "Suspending of solid particles in liquid by agitators," Chem. Eng. Sci., vol. 8, no. 3-4, pp. 244-253, 1958.

[22] P. M. Armenante and E. U. Nagamine, "Effect of low off-bottom impeller clearance on the minimum agitation speed for complete suspension of solids in stirred tanks," Chem. Eng. Sci., vol. 53, no. 9, pp. 1757-1775, 1998.

[23] A. W. Nienow, "Suspension of solid particles in turbine agitated baffled vessels," Chem. Eng. Sci., vol. 23, no. 12, pp. 1453-1459, 1968. 
[24] A. P. Van der Westhuizen and D. A. Deglon, "Solids suspension in a pilot-scale mechanical flotation cell: A critical impeller speed correlation," Miner. Eng., vol. 21, no. 8, pp. 621-629, 2008.

[25] K. J. Bittorf and S. M. Kresta, "Prediction of cloud height for solid suspensions in stirred tanks," Chem. Eng. Res. Des., vol. 81, no. 5, pp. 568-577, 2003.

[26] A. H. P. Skelland, Non-Newtonian flow and heat transfer. Wiley, 1967.

[27] G. B. Tatterson, Fluid mixing and gas dispersion in agitated tanks. McGraw-Hill Companies, 1991.

[28] C. R. Shervin, D. A. Raughley, and R. A. Romaszewski, "Flow visualization scaleup studies for the mixing of viscoelastic fluids," Chem. Eng. Sci., vol. 46, no. 11, pp. 2867-2873, 1991.

[29] K. Raghav Rao and J. B. Joshi, "Liquid phase mixing in mechanically agitated vessels," Chem. Eng. Commun., vol. 74, no. 1, pp. 1-25, 1988.

[30] M. F. W. Distelhoff, A. J. Marquis, J. M. Nouri, and J. H. Whitelaw, "Scalar mixing measurements in batch operated stirred tanks," Can. J. Chem. Eng., vol. 75, no. 4, pp. 641-652, 1997.

[31] P. A. Shamlou and E. Koutsakos, "Solids suspension and distribution in liquids under turbulent agitation," Chem. Eng. Sci., vol. 44, no. 3, pp. 529-542, 1989.

[32] J. C. Godfrey and Z. M. Zhu, "Measurement of particle-liquid profiles in agitated tanks," in AIChE Symposium Series, 1994, vol. 90, pp. 181-185.

[33] S. L. McKee, R. A. Williams, and A. Boxman, "Development of solid-liquid mixing models using tomographic techniques," Chem. Eng. J. Biochem. Eng. J., vol. 56, no. 3, pp. 101-107, 1995. 
[34] C. T. Crowe, Multiphase flow handbook, vol. 59. CRC press, 2005.

[35] T. Dyakowski, L. F. Jeanmeure, and A. J. Jaworski, "Applications of electrical tomography for gas-solids and liquid-solids flows—a review," Powder Technol., vol. 112, no. 3, pp. 174-192, 2000.

[36] Y. A. Wahab et al., "Non-invasive process tomography in chemical mixtures-A review," Sens. Actuators B Chem., vol. 210, pp. 602-617, 2015.

[37] H. S. Tapp and R. A. Williams, "Status and applications of microelectrical resistance tomography," Chem. Eng. J., vol. 77, no. 1, pp. 119-125, 2000.

[38] M. S. Beck, Process tomography: principles, techniques and applications. Butterworth-Heinemann, 2012.

[39] ITS, "P2000 Electrical Resistance Tomography System ITS System 2000 Version 5.0 Software Operating Manual." Industrial Tomography Systems Itd., 2005.

[40] R. Mann et al., "Application of electrical resistance tomography to interrogate mixing processes at plant scale," Chem. Eng. Sci., vol. 52, no. 13, pp. 2087-2097, 1997.

[41] P. A. D. T. Pinheiro, "Investigation of forward problem solvers and data collection methods in electrical impedance tomography," 1994.

[42] F. Dickin and M. Wang, "Electrical resistance tomography for process applications," Meas. Sci. Technol., vol. 7, no. 3, p. 247, 1996.

[43] W. R. Breckon and M. K. Pidcock, "Data errors and reconstruction algorithms in electrical impedance tomography," Clin. Phys. Physiol. Meas., vol. 9, no. 4A, p. 105, 1988. 
[44] Q. Wang, H. Wang, Z. Cui, Y. Xu, and C. Yang, "Fast reconstruction of electrical resistance tomography (ERT) images based on the projected CG method," Flow Meas. Instrum., vol. 27, pp. 37-46, 2012.

[45] R. A. Williams, X. Jia, and S. L. McKee, "Development of slurry mixing models using resistance tomography," Powder Technol., vol. 87, no. 1, pp. 21-27, 1996.

[46] R. Stevenson, S. T. Harrison, M. D. Mantle, A. J. Sederman, T. L. Moraczewski, and M. L. Johns, "Analysis of partial suspension in stirred mixing cells using both MRI and ERT," Chem. Eng. Sci., vol. 65, no. 4, pp. 1385-1393, 2010.

[47] C. Carletti, G. Montante, T. Westerlund, and A. Paglianti, "Analysis of solid concentration distribution in dense solid-liquid stirred tanks by electrical resistance tomography," Chem. Eng. Sci., vol. 119, pp. 53-64, 2014.

[48] P. Mishra and F. Ein-Mozaffari, "Using tomograms to assess the local solid concentrations in a slurry reactor equipped with a Maxblend impeller," Powder Technol., vol. 301, pp. 701-712, 2016.

[49] S. Luding, "Collisions \& contacts between two particles," in Physics of dry granular media, Springer, 1998, pp. 285-304.

[50] S. Luding, "Introduction to discrete element methods: basic of contact force models and how to perform the micro-macro transition to continuum theory," Eur. J. Environ. Civ. Eng., vol. 12, no. 7-8, pp. 785-826, 2008.

[51] H. R. Norouzi, N. Mostoufi, R. Zarghami, and R. Sotudeh-Gharebagh, Coupled CFDDEM Modeling: Formulation, Implementation and Application to Multiphase Flows. John Wiley \& Sons, 2016. 
[52] C. S. Campbell and C. E. Brennen, "Computer simulation of granular shear flows," J. Fluid Mech., vol. 151, pp. 167-188, 1985.

[53] N. G. Deen, M. V. S. Annaland, M. A. Van der Hoef, and J. A. M. Kuipers, "Review of discrete particle modeling of fluidized beds," Chem. Eng. Sci., vol. 62, no. 1, pp. 28-44, 2007.

[54] P. A. Cundall and O. D. Strack, "A discrete numerical model for granular assemblies," Geotechnique, vol. 29, no. 1, pp. 47-65, 1979.

[55] C. O'Sullivan, Particulate Discrete Element Modelling: A Geomechanics Perspective. Applied Geotechnics. Spon Press/Taylor \& Francis, 2011.

[56] P. A. Langston, U. Tüzün, and D. M. Heyes, "Discrete element simulation of granular flow in 2D and 3D hoppers: dependence of discharge rate and wall stress on particle interactions," Chem. Eng. Sci., vol. 50, no. 6, pp. 967-987, 1995.

[57] H. Hertz, "Über die Berührung fester elastischer Körper.," J. Für Reine Angew. Math., vol. 92, pp. 156-171, 1882.

[58] R. D. Mindlin and H. Deresiewicz, "Elastic spheres in contact under varying oblique forces," J. Appl. Mech., vol. 20, 1953.

[59] Y. Tsuji, T. Kawaguchi, and T. Tanaka, "Discrete particle simulation of twodimensional fluidized bed," Powder Technol., vol. 77, no. 1, pp. 79-87, 1993.

[60] O. R. Walton and R. L. Braun, "Viscosity, granular-temperature, and stress calculations for shearing assemblies of inelastic, frictional disks," J. Rheol. 1978Present, vol. 30, no. 5, pp. 949-980, 1986.

[61] C. Thornton and K. K. Yin, "Impact of elastic spheres with and without adhesion," Powder Technol., vol. 65, no. 1-3, pp. 153-166, 1991. 
[62] EDEM Solutions, “EDEM 1.3 User's Manual," Edinb. UK, 2006.

[63] Y. Sheng, C. J. Lawrence, B. J. Briscoe, and C. Thornton, "Numerical studies of uniaxial powder compaction process by 3D DEM," Eng. Comput., vol. 21, no. 2/3/4, pp. 304-317, 2004.

[64] C. O'Sullivan and J. D. Bray, "Modified shear spring formulation for discontinuous deformation analysis of particulate media," J. Eng. Mech., vol. 129, no. 7, pp. 830834, 2003.

[65] X. He and L.-S. Luo, "Theory of the lattice Boltzmann method: From the Boltzmann equation to the lattice Boltzmann equation," Phys. Rev. E, vol. 56, no. 6, p. 6811, 1997.

[66] A. P. Kothari, J. D. Anderson, and E. Jones, "Navier-Stokes solutions for chemical laser flows," AIAA J., vol. 15, no. 1, pp. 92-100, 1977.

[67] S. Succi, The lattice Boltzmann equation: for fluid dynamics and beyond. Oxford university press, 2001.

[68] J. Bernsdorf, F. Durst, and M. Schäfer, "Comparison of cellular automata and finite volume techniques for simulation of incompressible flows in complex geometries," Int. J. Numer. Methods Fluids, vol. 29, no. 3, pp. 251-264, 1999.

[69] J. Blazek, Computational fluid dynamics: principles and applications. ButterworthHeinemann, 2015.

[70] S. V. Patankar and D. B. Spalding, "A calculation procedure for heat, mass and momentum transfer in three-dimensional parabolic flows," Int. J. Heat Mass Transf., vol. 15 , no. 10 , pp. $1787-1806,1972$. 
[71] J. P. Van Doormaal and G. D. Raithby, "Enhancements of the SIMPLE method for predicting incompressible fluid flows," Numer. Heat Transf., vol. 7, no. 2, pp. 147$163,1984$.

[72] R. I. Issa, "Solution of the implicitly discretised fluid flow equations by operatorsplitting," J. Comput. Phys., vol. 62, no. 1, pp. 40-65, 1986.

[73] ANSYS, Manual and User Guide of Fluent Software, FLUENT. Inc, 2005.

[74] B. E. Launder and D. B. Spalding, "Lectures in mathematical models of turbulence," 1972.

[75] S. A. Orszag et al., "Renormalization group modeling and turbulence simulations," Wall Turbul. Flows, pp. 1031-1046, 1993.

[76] T.-H. Shih, W. W. Liou, A. Shabbir, Z. Yang, and J. Zhu, "A new k-epsilon eddy viscosity model for high Reynolds number turbulent flows: Model development and validation," 1994.

[77] K. Hanjalic and B. E. Launder, "A Reynolds stress model of turbulence and its application to thin shear flows," J. Fluid Mech., vol. 52, no. 04, pp. 609-638, 1972.

[78] J. Tu, G. H. Yeoh, and C. Liu, Computational fluid dynamics: a practical approach. Butterworth-Heinemann, 2012.

[79] E. Loth, "Numerical approaches for motion of dispersed particles, droplets and bubbles," Prog. Energy Combust. Sci., vol. 26, no. 3, pp. 161-223, 2000.

[80] D. A. Drew and S. L. Passman, Theory of multicomponent fluids, vol. 135. Springer Science \& Business Media, 2006.

[81] B. K. Cook, D. R. Noble, and J. R. Williams, "A direct simulation method for particlefluid systems," Eng. Comput., vol. 21, no. 2/3/4, pp. 151-168, 2004. 
[82] H. H. Hu, "Direct simulation of flows of solid-liquid mixtures," Int. J. Multiph. Flow, vol. 22, no. 2, pp. 335-352, 1996.

[83] N. Sharma, Y. Chen, and N. A. Patankar, "A distributed lagrange multiplier based computational method for the simulation of particulate-Stokes flow," Comput. Methods Appl. Mech. Eng., vol. 194, no. 45, pp. 4716-4730, 2005.

[84] A. Prosperetti and H. N. Oguz, "Physalis: a new o (N) method for the numerical simulation of disperse systems: potential flow of spheres," J. Comput. Phys., vol. 167, no. 1, pp. 196-216, 2001.

[85] T. B. Anderson and R. Jackson, "Fluid mechanical description of fluidized beds. equations of motion," Ind. Eng. Chem. Fundam., vol. 6, no. 4, pp. 527-539, 1967.

[86] B. H. Xu and A. B. Yu, "Numerical simulation of the gas-solid flow in a fluidized bed by combining discrete particle method with computational fluid dynamics," Chem. Eng. Sci., vol. 52, no. 16, pp. 2785-2809, 1997.

[87] D. Gidaspow, Multiphase flow and fluidization: continuum and kinetic theory descriptions. Academic press, 1994.

[88] Z. Y. Zhou, S. B. Kuang, K. W. Chu, and A. B. Yu, "Discrete particle simulation of particle-fluid flow: model formulations and their applicability," J. Fluid Mech., vol. 661, pp. 482-510, 2010.

[89] F. Odar and W. S. Hamilton, "Forces on a sphere accelerating in a viscous fluid," J. Fluid Mech., vol. 18, no. 02, pp. 302-314, 1964.

[90] M. R. Maxey and J. J. Riley, "Equation of motion for a small rigid sphere in a nonuniform flow," Phys. Fluids 1958-1988, vol. 26, no. 4, pp. 883-889, 1983. 
[91] P. G. T. Saffman, "The lift on a small sphere in a slow shear flow," J. Fluid Mech., vol. 22, no. 02, pp. 385-400, 1965.

[92] T. Shao, Y. Hu, W. Wang, Y. Jin, and Y. Cheng, "Simulation of Solid Suspension in a Stirred Tank Using CFD-DEM Coupled Approach," Chin. J. Chem. Eng., vol. 21, no. 10, pp. 1069-1081, Oct. 2013.

[93] S. Wang, S. Guo, J. Gao, X. Lan, Q. Dong, and X. Li, "Simulation of flow behavior of liquid and particles in a liquid-solid fluidized bed," Powder Technol., vol. 224, pp. 365-373, 2012.

[94] R. Di Felice, "The voidage function for fluid-particle interaction systems," Int. J. Multiph. Flow, vol. 20, no. 1, pp. 153-159, 1994.

[95] M. Syamlal and T. J. O'Brien, "Computer simulation of bubbles in a fluidized bed," in AIChE Symp. Ser, 1989, vol. 85, pp. 22-31.

[96] A. T. Hjelmfelt Jr and L. F. Mockros, "Motion of discrete particles in a turbulent fluid," Appl. Sci. Res., vol. 16, no. 1, pp. 149-161, 1966.

[97] W. Zhang, R. Noda, and M. Horio, "Evaluation of lubrication force on colliding particles for DEM simulation of fluidized beds," Powder Technol., vol. 158, no. 1, pp. 92-101, 2005.

[98] J. J. Derksen, "Numerical simulation of solids suspension in a stirred tank," AIChE J., vol. 49, no. 11, pp. 2700-2714, 2003.

[99] S. Elghobashi, "On predicting particle-laden turbulent flows," Appl. Sci. Res., vol. 52, no. 4, pp. 309-329, 1994.

[100] Itasca, "PFC3D Particle Flow Code in 3 Dimensions CCFD Add-on." Itasca Consulting Group Inc., 2008. 
[101] A. Hobbs, "Simulation of an aggregate dryer using coupled CFD and DEM methods," Int. J. Comput. Fluid Dyn., vol. 23, no. 2, pp. 199-207, 2009.

[102] B. Blais, M. Lassaigne, C. Goniva, L. Fradette, and F. Bertrand, "Development of an unresolved CFD-DEM model for the flow of viscous suspensions and its application to solid-liquid mixing," J. Comput. Phys., vol. 318, pp. 201-221, 2016.

[103] B. Blais and F. Bertrand, "CFD-DEM investigation of viscous solid-liquid mixing: impact of particle properties and mixer characteristics," Chem. Eng. Res. Des., 2016.

[104] Y. Xia, F. F. Peng, and E. Wolfe, "CFD simulation of fine coal segregation and stratification in jigs," Int. J. Miner. Process., vol. 82, no. 3, pp. 164-176, 2007.

[105] T. Zhao, G. T. Houlsby, and S. Utili, "Investigation of granular batch sedimentation via DEM-CFD coupling," Granul. Matter, vol. 16, no. 6, pp. 921-932, 2014.

[106] S. M. Viduka, Y. Q. Feng, K. Hapgood, and M. P. Schwarz, "Discrete particle simulation of solid separation in a jigging device," Int. J. Miner. Process., vol. 123, pp. 108-119, 2013.

[107] S. Viduka, Y. Feng, K. Hapgood, and P. Schwarz, "CFD-DEM investigation of particle separations using a sinusoidal jigging profile," Adv. Powder Technol., vol. 24, no. 2, pp. 473-481, 2013.

[108] S. M. Viduka, Y. Q. Feng, K. Hapgood, and M. P. Schwarz, "CFD-DEM linvestigation of particle separations using a trapezoidal jigging profile," in Ninth International Conference on CFD in the Minerals and Process Industries, CSIRO, 2012, pp. 1-8. 
[109] S. Huang, X. Su, and G. Qiu, "Transient numerical simulation for solid-liquid flow in a centrifugal pump by DEM-CFD coupling," Eng. Appl. Comput. Fluid Mech., vol. 9, no. 1, pp. 411-418, 2015.

[110] C. Kloss, C. Goniva, A. Hager, S. Amberger, and S. Pirker, "Models, algorithms and validation for opensource DEM and CFD-DEM," Prog. Comput. Fluid Dyn. Int. J., vol. 12, no. 2-3, pp. 140-152, 2012.

[111] C. Kloss, C. Goniva, G. Aichinger, and S. Pirker, "Comprehensive DEM-DPM-CFD simulations-model synthesis, experimental validation and scalability," in Proceedings of the Seventh International Conference on CFD in the Minerals and Process Industries, CSIRO, Melbourne, Australia, 2009.

[112] X. R. Fernández and H. Nirschl, "Simulation of particles and sediment behaviour in centrifugal field by coupling CFD and DEM," Chem. Eng. Sci., vol. 94, pp. 7-19, 2013.

[113] J. Chen, Y. Wang, X. Li, R. He, S. Han, and Y. Chen, "Reprint of 'Erosion prediction of liquid-particle two-phase flow in pipeline elbows via CFD-DEM coupling method,"' Powder Technol., vol. 282, pp. 25-31, 2015.

[114] C. L. Wu, O. Ayeni, A. S. Berrouk, and K. Nandakumar, "Parallel algorithms for CFD-DEM modeling of dense particulate flows," Chem. Eng. Sci., vol. 118, pp. 221244, 2014.

[115] M. Varga, C. Goniva, K. Adam, and E. Badisch, "Combined experimental and numerical approach for wear prediction in feed pipes," Tribol. Int., vol. 65, pp. 200206, 2013. 
[116] Q. Han, N. Yang, J. Zhu, and M. Liu, "Onset velocity of circulating fluidization and particle residence time distribution: A CFD-DEM study," Particuology, vol. 21, pp. 187-195, 2015.

[117] F. Cello, A. Di Renzo, and F. P. Di Maio, "DEM-CFD simulations of layer inversion in two-component liquid fluidized beds," 2009.

[118] A. Di Renzo, F. Cello, and F. P. Di Maio, "Simulation of the layer inversion phenomenon in binary liquid--fluidized beds by DEM-CFD with a drag law for polydisperse systems," Chem. Eng. Sci., vol. 66, no. 13, pp. 2945-2958, 2011.

[119] A. Di Renzo and F. P. Di Maio, "Homogeneous and bubbling fluidization regimes in DEM-CFD simulations: hydrodynamic stability of gas and liquid fluidized beds," Chem. Eng. Sci., vol. 62, no. 1, pp. 116-130, 2007.

[120] K. J. Dong, S. B. Kuang, A. Vince, T. Hughes, and A. B. Yu, "Numerical simulation of the in-line pressure jig unit in coal preparation," Miner. Eng., vol. 23, no. 4, pp. 301-312, 2010.

[121] P. U. Foscolo and L. G. Gibilaro, "Fluid dynamic stability of fluidised suspensions: the particle bed model," Chem. Eng. Sci., vol. 42, no. 6, pp. 1489-1500, 1987.

[122] L. G. Gibilaro, Fluidization dynamics. Butterworth-Heinemann, 2001.

[123] G. Liu, F. Yu, H. Lu, S. Wang, P. Liao, and Z. Hao, "CFD-DEM simulation of liquidsolid fluidized bed with dynamic restitution coefficient," Powder Technol., vol. 304, pp. 186-197, 2016.

[124] Z. Peng, S. V. Ghatage, E. Doroodchi, J. B. Joshi, G. M. Evans, and B. Moghtaderi, "Forces acting on a single introduced particle in a solid-liquid fluidised bed," Chem. Eng. Sci., vol. 116, pp. 49-70, 2014. 
[125] K. F. Malone, B. H. Xu, and M. Fairweather, "Numerical Investigation of the LayerInversion Phenomenon in Binary Solid Liquid Fluidized Beds," 2007.

[126] F. P. Di Maio, A. Di Renzo, and F. Cello, "A new drag model for detailed simulations of fluid-particle systems.," presented at the MFIP 2008 - 11th International Conference on Multiphase Flow in Industrial Plant, 2008, pp. 243-250.

[127] F. Cello, A. Di Renzo, and F. P. Di Maio, "A semi-empirical model for the drag force and fluid-particle interaction in polydisperse suspensions," Chem. Eng. Sci., vol. 65, no. 10, pp. 3128-3139, 2010.

[128] S. Al-Arkawazi, C. Marie, K. Benhabib, and P. Coorevits, "Modeling the hydrodynamic forces between fluid-granular medium by coupling DEM-CFD," Chem. Eng. Res. Des., vol. 117, pp. 439-447, 2017.

[129] K. F. Malone and B. H. Xu, "Determination of contact parameters for discrete element method simulations of granular systems," Particuology, vol. 6, no. 6, pp. 521-528, 2008.

[130] J. D. Oroná, S. E. Zorrilla, and J. M. Peralta, "Computational fluid dynamics combined with discrete element method and discrete phase model for studying a food hydrofluidization system," Food Bioprod. Process., vol. 102, pp. 278-288, 2017.

[131] S. Wang, Z. Sun, X. Li, J. Gao, X. Lan, and Q. Dong, "Simulation of flow behavior of particles in liquid-solid fluidized bed with uniform magnetic field," Powder Technol., vol. 237, pp. 314-325, 2013.

[132] S. Wang et al., "Study of hydrodynamic characteristics of particles in liquid-solid fluidized bed with uniform transverse magnetic field," Powder Technol., vol. 245, pp. 314-323, 2013. 
[133] E. W. C. Lim, "Voidage waves in hydraulic conveying through narrow pipes," Chem. Eng. Sci., vol. 62, no. 17, pp. 4529-4543, 2007.

[134] A. Bravo-Blanco, A. Sánchez-Medina, and F. Ayuga, "Analysis of the incipient motion of spherical particles in an open channel bed, using a coupled computational fluid dynamics-discrete element method model," Biosyst. Eng., vol. 155, pp. 68-76, 2017.

[135] J. D. Redlinger-Pohn, L. M. König, C. Kloss, C. Goniva, and S. Radl, "Modelling of Non-Spherical, Elongated Particles for Industrial Suspension Flow Simulation," in VII European Congress on Computational Methods in Applied Sciences and Engineering. Crete Island, 2016, p. 14.

[136] G. G. Joseph, R. Zenit, M. L. Hunt, and A. M. Rosenwinkel, "Particle-wall collisions in a viscous fluid," J. Fluid Mech., vol. 433, pp. 329-346, 2001.

[137] I. Tomac and M. Gutierrez, "Fluid lubrication effects on particle flow and transport in a channel," Int. J. Multiph. Flow, vol. 65, pp. 143-156, 2014.

[138] Y. Tan, H. Zhang, D. Yang, S. Jiang, J. Song, and Y. Sheng, "Numerical simulation of concrete pumping process and investigation of wear mechanism of the piping wall," Tribol. Int., vol. 46, no. 1, pp. 137-144, 2012.

[139] H. Zhang et al., "Numerical investigation of the location of maximum erosive wear damage in elbow: Effect of slurry velocity, bend orientation and angle of elbow," Powder Technol., vol. 217, pp. 467-476, 2012.

[140] M. Azimian, M. Lichti, and H. J. Bart, "Investigation of Particulate Flow in a Channel by Application of CFD, DEM and LDA/PDA," Open Chem. Eng. J., vol. 8, pp. 1-11, 2014. 
[141] K. J. Dong, R. Y. Yang, R. P. Zou, A. B. Yu, G. Roach, and E. Jamieson, "Simulation of the cake formation and growth in sedimentation and filtration," in Third International Conference on CFD in the Minerals and Process Industries, CSIRO, Melbourne, Australia, 2003, pp. 10-12.

[142] K. J. Dong, R. Y. Yang, R. P. Zou, and A. B. Yu, "Role of interparticle forces in the formation of random loose packing," Phys. Rev. Lett., vol. 96, no. 14, p. 145505, 2006.

[143] K. J. Dong, R. P. Zou, R. Y. Yang, A. B. Yu, and G. Roach, "DEM simulation of cake formation in sedimentation and filtration," Miner. Eng., vol. 22, no. 11, pp. 921930, 2009.

[144] S. Sören and T. Jürgen, "Simulation of a Filtration Process by DEM and CFD," J. ISSN, vol. 1929, p. 2724, 2012.

[145] K. W. Chu, B. Wang, A. B. Yu, and A. Vince, "CFD-DEM modelling of multiphase flow in dense medium cyclones," Powder Technol., vol. 193, no. 3, pp. 235-247, 2009.

[146] K. W. Chu, B. Wang, A. B. Yu, A. Vince, G. D. Barnett, and P. J. Barnett, "CFDDEM study of the effect of particle density distribution on the multiphase flow and performance of dense medium cyclone," Miner. Eng., vol. 22, no. 11, pp. 893-909, 2009.

[147] X. R. Fernandez, L. E. Spelter, and H. Nirschl, "Computational Fluid Dynamics (CFD) and Discrete Element Method (DEM) Applied to Centrifuges," in Applied Computational Fluid Dynamics, InTech, 2012. 
[148] Y. K. Xia and F. F. Peng, "Numerical simulation of behavior of fine coal in oscillating flows," Miner. Eng., vol. 20, no. 2, pp. 113-123, 2007.

[149] R. Misumi, T. Sasaki, H. Kato, K. Nishi, and M. Kaminoyama, "Relation between particle rising behavior and liquid flow around the bottom of a stirred vessel," in 14th European Conference on Mixing, Session V, 2012, pp. 299-304.

[150] T. Srinivasa and S. Jayanti, "An Eulerian/Lagrangian study of solid suspension in stirred tanks," AIChE J., vol. 53, no. 9, pp. 2461-2469, 2007.

[151] D. Boucher, Z. Deng, T. Leadbeater, R. Langlois, and K. E. Waters, "Observation of iron ore beneficiation within a spiral concentrator by positron emission particle tracking of large $(\varnothing=1440 \mu \mathrm{m})$ and small $(\varnothing=58 \mu \mathrm{m})$ hematite and quartz tracers," Chem. Eng. Sci., vol. 140, pp. 217-232, 2016.

[152] B. Blais, O. Bertrand, L. Fradette, and F. Bertrand, "CFD-DEM simulations of early turbulent solid-liquid mixing: Prediction of suspension curve and just-suspended speed," Chem. Eng. Res. Des., 2017.

[153] D. C. Barber and A. D. Seagar, "Fast reconstruction of resistance images," Clin. Phys. Physiol. Meas., vol. 8, no. 4A, p. 47, 1987.

[154] C. J. Kotre, "A sensitivity coefficient method for the reconstruction of electrical impedance tomograms," Clin. Phys. Physiol. Meas., vol. 10, no. 3, p. 275, 1989.

[155] J. R. Bourne and R. N. Sharma, "Homogeneous particle suspension in propelleragitated flat bottomed tanks," Chem. Eng. J., vol. 8, no. 3, pp. 243-250, 1974.

[156] L. Musil, "The hydrodynamics of mixed crystallizers," Collect. Czechoslov. Chem. Commun., vol. 41, no. 3, pp. 839-852, 1976. 
[157] L. Musil and J. VIk, "Suspending solid particles in an agitated conical-bottom tank," Chem. Eng. Sci., vol. 33, no. 8, pp. 1123-1131, 1978.

[158] Y. Tsuji, T. Tanaka, and T. Ishida, "Lagrangian numerical simulation of plug flow of cohesionless particles in a horizontal pipe," Powder Technol., vol. 71, no. 3, pp. 239-250, 1992.

[159] A. Di Renzo and F. P. Di Maio, "Comparison of contact-force models for the simulation of collisions in DEM-based granular flow codes," Chem. Eng. Sci., vol. 59, no. 3, pp. 525-541, 2004.

[160] S. Olivieri, F. Picano, G. Sardina, D. ludicone, and L. Brandt, "The effect of the Basset history force on particle clustering in homogeneous and isotropic turbulence," Phys. Fluids, vol. 26, no. 4, p. 041704, 2014.

[161] M. van Aartrijk and H. J. H. Clercx, "Vertical dispersion of light inertial particles in stably stratified turbulence: The influence of the Basset force," Phys. Fluids 1994Present, vol. 22, no. 1, p. 013301, 2010.

[162] M. A. T. Van Hinsberg, J. H. M. ten Thije Boonkkamp, and H. J. H. Clercx, "An efficient, second order method for the approximation of the Basset history force," J. Comput. Phys., vol. 230, no. 4, pp. 1465-1478, 2011.

[163] N.-F. Chang, C.-Y. Chiang, T.-C. Chen, and L.-G. Chen, "Cubic spline interpolation with overlapped window and data reuse for on-line hilbert huang transform biomedical microprocessor," in Engineering in Medicine and Biology Society, EMBC, 2011 Annual International Conference of the IEEE, 2011, pp. 7091-7094.

[164] H. Ihshaish, A. Cortés, and M. Senar, "Genetic ensemble (G-Ensemble) for meteorological prediction enhancement," 2011. 
[165] Q. Zou and Y. Luo, "Biophysical characterization for product comparability," BioPharm, p. 8, 2010.

[166] M. Ebrahimi, "CFD-DEM modelling of two-phase pneumatic conveying with experimental validation.," 2014.

[167] J. Lundberg and B. M. Halvorsen, "A review of some exsisting drag models describing the interaction between phases in a bubbling fluidized bed," in Proc. 49th Scand. Conf. Simulation and Modeling, Oslo University College, Oslo, Norway, 2008, pp. $7-8$.

[168] M. A. T. van Hinsberg, H. J. H. Clercx, and F. Toschi, "Enhanced settling of nonheavy inertial particles in homogeneous isotropic turbulence: The role of the pressure gradient and the Basset history force," Phys. Rev. E, vol. 95, no. 2, p. 023106, 2017.

[169] A. Yousefi and T.-T. Ng, "Dimensionless input parameters in discrete element modeling and assessment of scaling techniques," Comput. Geotech., vol. 88, pp. 164-173, 2017. 